coloproctology $2017 \cdot 39: 16-66$ DOI 10.1007/s00053-016-0110-7

Online publiziert: 5. Dezember 2016

(c) Springer-Verlag Berlin Heidelberg 2016

CrossMark
A. Ommer ${ }^{1}$ A. Herold ${ }^{2}$ E. Berg B S. Farke $^{4}$ A. Fürst ${ }^{5} \cdot$ F. Hetzer ${ }^{6} \cdot$ A. Köhler ${ }^{7}$. S. Post ${ }^{8} \cdot$ R. Ruppert ${ }^{9} \cdot$ M. Sailer ${ }^{10} \cdot$ T. Schiedeck $^{11} \cdot 0$. Schwandner $^{12}$.

B. Strittmatter ${ }^{13} \cdot$ B. H. Lenhard ${ }^{14} \cdot$ W. Bader ${ }^{15} \cdot$ S. Krege ${ }^{16} \cdot$ H. Krammer ${ }^{17} \cdot$ E. Stange $^{18}$

'End- und Dickdarmdarm-Zentrum Essen, Essen, Deutschland

${ }^{2}$ Enddarmzentrum Mannheim, Mannheim, Deutschland

${ }^{3}$ Prosper Hospital, Recklinghausen, Deutschland

${ }^{4}$ Klinikum Delmenhorst, Delmenhorst, Deutschland

${ }^{5}$ Caritas Krankenhaus, Regensburg, Deutschland

${ }^{6}$ Spital Linth, Uznach, Schweiz

${ }^{7}$ HELIOS St. Johannes Klinik Duisburg, Duisburg, Deutschland

${ }^{8}$ Universitätsklinik Mannheim, Mannheim, Deutschland

${ }^{9}$ Klinikum Neuperlach, Städt. Klinikum München $\mathrm{GmbH}$, München, Deutschland

${ }^{10}$ Bethesda-Krankenhaus, Hamburg, Deutschland

${ }^{11}$ Klinikum Ludwigsburg, Ludwigsburg, Deutschland

${ }^{12}$ Krankenhaus Barmherzige Brüder, Regensburg, Deutschland

${ }^{13}$ Praxis für Koloproktologie, Praxisklinik 2000, Freiburg, Deutschland

${ }^{14}$ Praxis für Enddarmerkrankungen, Heidelberg, Deutschland

${ }^{15}$ Klinikum Bielefeld, Bielefeld, Deutschland

${ }^{16}$ Kliniken Essen-Mitte, Essen, Deutschland

${ }^{17}$ Gastroenterologie am End-Darm-Zentrum, Mannheim, Deutschland

${ }^{18}$ Robert-Bosch-Krankenhaus, Stuttgart, Deutschland

\section{S3-Leitlinie: Kryptoglanduläre Analfisteln}

\section{2. revidierte Fassung, AWMF-Registriernummer: 088/003}

\section{Einleitung}

Das anale Fistelleiden ist ein häufiges Krankheitsbild. Die Symptomatik besteht in Schmerzen und Sekretion aus einer Öffnung. Bei der kryptoglandulären Analfistel besteht eine Verbindung zwischen einer Öffnung auf Höhe der Linea dentata und einer Öffnung in der Perianalregion. Die häufigste Ursache für die Entstehung von Analfisteln ist die Entzündung der beim Menschen rudimentär angelegten Proktodealdrüsen (sog. kryptoglanduläre Analfistel). Dabei stellt der Analabszess das akute und die Analfistel das chronische Stadium des gleichen Krankheitsbildes dar. Bezüglich des Analabszesses wird auf die entsprechende Leitlinie verwiesen.

Von dieser Krankheitsgenese sind Analfisteln zu unterscheiden, deren Ursache nicht kryptoglandulären Ur- sprungs ist. Hierzu zählen: chronischentzündliche Darmerkrankungen (z. B. Morbus Crohn, Divertikulitis), bakterielle Infektionen (z. B. Tuberkulose), immunsupprimierende Erkrankungen (z. B. HIV, Leukämie), Malignome und Zustände nach operativer Behandlung im Bereich des Beckenbodens (z. B. tiefe anteriore Rektumresektion). Weitere Entitäten stellen der Pilonidalsinus und die Acne inversa dar, deren subkutan verlaufende Fisteln keine Verbindung zum Analkanal aufweisen und somit nicht den Analfisteln zuzuordnen sind. Auch hier wird auf die entsprechenden Leitlinien verwiesen. Selten sind Fisteln durch chirurgische Eingriffe [190] und vergessene operative Fremdkörper [305]. Diese sind grundsätzlich nicht Bestandteil dieser Leitlinie, obwohl sich die Therapie nach den beschriebenen Richtlinien richten sollte.

\section{Beteiligte Fachgesellschaften}

- Deutsche Gesellschaft für Allgemein- und Viszeralchirurgie (DGAV)

- Chirurgische Arbeitsgemeinschaft für Coloproktologie (CACP)

- Deutsche Gesellschaft für Koloproktologie (DGK)

- Berufsverband der Coloproktologen Deutschlands (BCD)

- Deutsche Gesellschaft für Gastroenterologie, Verdauungs- und Stoffwechselkrankheiten (DGVS)

- Deutsche Dermatologische Gesellschaft (DDG)

- Deutsche Gesellschaft für Urologie (DGU)

- Arbeitsgemeinschaft für Urogynäkologie und plastische Beckenbodenrekonstruktion e. V. (AGUB) der Deutschen Gesellschaft für Gynäkologie und Geburtshilfe (DGGG) 
Tab. 1 Definitionen von Evidenzstärke und Empfehlungsgrad in Anlehnung an die Empfehlungen des Centre for Evidence-Based Medicine, Oxford, UK [191]

\begin{tabular}{|c|c|c|}
\hline $\begin{array}{l}\text { Empfehlungs- } \\
\text { grad }\end{array}$ & $\begin{array}{l}\text { Evidenz- } \\
\text { level }\end{array}$ & Typen von Therapiestudien \\
\hline$A\left({ }_{, \prime}\right.$ soll $\left.{ }^{\prime \prime}\right)$ & $\begin{array}{l}1 \mathrm{a} \\
1 \mathrm{~b} \\
1 \mathrm{c}\end{array}$ & $\begin{array}{l}\text { Systematisches Review randomisierter kontrollierter Studien (RCT) } \\
\text { Geeignete geplante RCT } \\
\text { Alles-oder-nichts-Prinzip }\end{array}$ \\
\hline B (,sollte") & $\begin{array}{l}2 a \\
2 b\end{array}$ & $\begin{array}{l}\text { Systematisches Review gut geplanter Kohortenstudien } \\
\text { Gut geplante Kohortenstudie, einschließlich RCT mit mäßigem } \\
\text { Follow-up (<80\%) }\end{array}$ \\
\hline $0($ („kann“) & $\begin{array}{l}3 a \\
3 b\end{array}$ & $\begin{array}{l}\text { Systematisches Review von gut geplanten Fall-Kontroll-Studien } \\
\text { Gut geplante Fall-Kontroll-Studie }\end{array}$ \\
\hline $0($ (,kann“) & 4 & $\begin{array}{l}\text { Fallserien, einschließlich schlechter Kohorten- und Fall-Kontroll-Stu- } \\
\text { dien }\end{array}$ \\
\hline $0($ („kann“) & 5 & $\begin{array}{l}\text { Meinungen ohne explizite kritische Bewertung, physiologische } \\
\text { Modelle, Vergleiche oder Grundsätze }\end{array}$ \\
\hline
\end{tabular}

\section{Methodik}

Die Erstellung der Leitlinie erfolgte grundsätzlich nur für Analfisteln auf dem Boden einer kryptoglandulären Infektion. Diese Leitlinie stellt eine komplett überarbeitete Neufassung der S3Leitlinie „Kryptoglanduläre Analfisteln“ aus dem Jahr 2011 [290] dar.

Primäre Berücksichtigung fanden die Leitlinien anderer Fachgesellschaften (American Society of Colon and Rectal Surgeons [480], Association of Coloproctology of Great Britain and Ireland [483], Italienische Gesellschaft für Kolorektale Chirurgie (SICCR; [21]), Europäische Gesellschaft für Coloproktologie [91] sowie eine Cochrane-Analyse [195]. Im Kapitel Reviews werden diese Aussagen noch einmal gesondert gewertet. Diese wurden im Rahmen der PubMed-Analyse erfasst. Die Aussagen dieser externen Leitlinien fanden lediglich als Publikationen Eingang in diese Leitlinien und wurden nicht als bindend angesehen. Daher fand keine methodische Bewertung der Leitlinie statt.

Grundlage der Revision war ein erneutes umfangreiches Review der Literatur unter Nutzung der Datenbanken PubMed und Cochrane mit den Stichworten „anal fistula“ oder „fistulain-ano“ und „201*“. Diese Recherche (Stichtag 29.11.2015) ergab 457 Treffer. Im Rahmen der weiteren Auswertung wurden zunächst Artikel, die anhand des Titels keine Relevanz für die Leitlinie hatten (chronisch-entzündliche Darmerkrankungen, rektovaginale Fis- teln, kindliche Fisteln, Textsprache nicht Englisch oder Deutsch u. a.), insgesamt 361 Arbeiten ausgeschlossen.

Von den übrig gebliebenen Arbeiten waren 25 bereits in der ersten Fassung berücksichtigt worden. Ergänzt wurde diese Literaturrecherche durch eine bereits in den Jahren 2011 bis 2015 durchgeführte kontinuierliche Aufarbeitung der relevanten Zeitschriften in Bezug auf relevante Artikel durch den federführenden Autor. Dabei wurden auch Publikationen aus nicht Pubmed-gelisteten und deutschsprachigen Zeitschriften (insbesondere coloproctology) erfasst.

Die auf diesem Weg identifizierten neuen Literaturstellen wurden bezüglich der im Folgenden definierten Fragestellungen zunächst anhand des Abstracts und ggf. auch des Volltextes evaluiert. In Bezug auf die einzelnen Kapitel der Leitlinie wurden die übrigen Literaturstellen bzgl. ihrer Aussage zu Epidemiologie und Ätiologie herausgefiltert. Bezüglich der Diagnostik wurden die Stichwörter „Klinische Untersuchung“, „Endosonographie“ und "Magnetresonanztomographie“ berücksichtigt. Hinsichtlich der möglichen Therapieoptionen wurden neben den randomisierten Studien alle Arbeiten ausgewertet, die Follow-upErgebnisse lieferten. In den Evidenztabellen wurden dabei folgende Ergebnisse der einzelnen Studien erfasst: Anzahl der behandelten Patienten, Fisteltyp, Anteil Morbus Crohn, Studiendesign (retro-/ prospektiv, randomisiert), Heilungsrate, Rezidivrate, Häufigkeit von Kontinenzstörungen sowie die Follow-up-Zeit.
Tab. 2 Klassifikation der Konsensusstärke

\begin{tabular}{|l|l|}
$\begin{array}{l}\text { Starker Kon- } \\
\text { sens }\end{array}$ & $\begin{array}{l}\text { Zustimmung von >95\% } \\
\text { der Teilnehmer }\end{array}$ \\
\hline Konsens & $\begin{array}{l}\text { Zustimmung von } \\
>75-95 \% \text { der Teilneh- } \\
\text { mer }\end{array}$ \\
\hline $\begin{array}{l}\text { Mehrheitliche } \\
\text { Zustimmung }\end{array}$ & $\begin{array}{l}\text { Zustimmung von } \\
>50-75 \% \text { der Teilneh- } \\
\text { mer }\end{array}$ \\
\hline Kein Konsens & $\begin{array}{l}\text { Zustimmung von <50\% } \\
\text { der Teilnehmer }\end{array}$ \\
\hline
\end{tabular}

Die Bewertung der vorliegenden Literatur zur Behandlung der Analfisteln erfolgte im Sinne eines methodenkritischen Lesens. Problematische Punkte in den meisten Publikationen waren dabei kleine Fallzahlen, retrospektive Aufarbeitung des Patientenguts sowie unvollständige Nachuntersuchung. Da die wenigen randomisierten Studien nur sehr unzureichend das Therapiespektrum wiedergeben oder gar vergleichen, mussten auch diese z.T. methodisch minderwertigen Arbeiten als Gesamtheit herangezogen werden, um eine Einschätzung der einzelnen Operationsverfahren vornehmen zu können.

Definitionen von Evidenzstärke und Empfehlungsgrad wurden in Anlehnung an die Empfehlungen des Centre for Evidence-Based Medicine, Oxford, UK (http://www.cebm.net/) anhand - Tab. 1 sowie - Abb. 1 und die Klassifikation der Konsensusstärke anhand - Tab. 2 festgelegt. Bei fehlender Evidenz wurde ein klinischer Konsensuspunkt (KKP) empfohlen als gute klinische Praxis („good clinical practice point") im Konsens und aufgrund der klinischen Erfahrungen der Mitglieder der Leitliniengruppe als ein Standard in der Behandlung, bei dem keine experimentelle wissenschaftliche Erforschung möglich oder angestrebt ist.

Die Ausarbeitung des Textes geschah im Rahmen einer Konsensuskonferenz aus Mitgliedern der beteiligten Fachgesellschaften (• Tab. 3), die am 11.03.2016 in München im Rahmen der Koloproktologietage 2016 stattfand. Die Vorbereitung und die Organisation wurden vom Koordinator (Dr. Andreas Ommer) übernommen. Die Konsensuskonferenz wurde durch den unabhängigen Moderator (Dr. Jan Jongen, Kiel) moderiert. Der unabhängige Moderator (Dr. Jan Jongen) 
coloproctology $2017 \cdot 39: 16-66 \quad$ DOI 10.1007/s00053-016-0110-7

(c) Springer-Verlag Berlin Heidelberg 2016

A. Ommer · A. Herold · E. Berg · S. Farke · A. Fürst · F. Hetzer · A. Köhler · S. Post · R. Ruppert · M. Sailer · T. Schiedeck · O. Schwandner · B. Strittmatter $\cdot$ B. H. Lenhard $\cdot$ W. Bader $\cdot$ S. Krege $\cdot$ H. Krammer $\cdot$ E. Stange

\section{S3-Leitlinie: Kryptoglanduläre Analfisteln. 2. revidierte Fassung, AWMF-Registriernummer: 088/003}

\section{Zusammenfassung}

Hintergrund. Kryptoglanduläre Analfisteln sind mit 2 pro 10.000 Einwohner/Jahr eine häufige Erkrankung mit einem Häufigkeitsgipfel bei jungen männlichen Erwachsenen. Eine nicht adäquate Behandlung kann zu einer Beeinträchtigung der Lebensqualität und insbesondere zur Reduktion der Kontinenzleistung führen.

Methode. Aufbauend auf der S3-Leitlinie aus dem Jahr 2011 wurde eine erneute systematische Literaturrecherche durchgeführt, und die Leitlinien wurden aktualisiert.

Ergebnis. Relevante randomisierte Studien sind weiterhin selten, so dass das Evidenzniveau weitgehend unverändert niedrig bleibt. Bezüglich Klassifikation und Diagnostik gelten die Empfehlungen der vorausgehenden Leitlinie unverändert. Zur Therapie der Analfistel stehen folgende operative Verfahren zur Verfügung: Fistelspaltung, Fadendrainage, plastische Rekonstruktion mit Sphinkternaht sowie Okklusion mit Biomaterialien. Als neue Technik wurde in mehreren Studien das LIFT-Verfahren vorgestellt. Die Ergebnisse bezüglich Heilungsraten und Kontinenzstörungen entsprechen weitgehend denen der plastischen Rekonstruktionstechniken. Die Bewertung weiterer neuer Verfahren (Laser, VAAFT, OTS-Clip) ist mangels repräsentativer Literatur derzeit nicht abschließend möglich. Eine Fistelspaltung sollte nur bei oberflächlichen Fisteln erfolgen. Die Gefahr der postoperativen Kontinenzstörung steigt mit der Menge des durchtrennten Sphinkteranteils. Bei allen hohen Analfisteln soll ein sphinkterschonendes Verfahren zur Anwendung kommen. Die Ergebnisse der verschiedenen Techniken zur plastischen
Rekonstruktion sind weitgehend identisch. Für die Okklusion durch Biomaterialien ist insgesamt eine deutlich geringere Heilungsrate zu konstatieren als primär berichtet. Auch hier ist der Stellenwert neuerer Materialien (neue Plugs, Kollagen, autologe Stammzellen) mangels Evidenz nicht abschließend möglich. Schlussfolgerung. In dieser S3-Leitlinie werden auf dem Boden der aktuellen Literatur evidenzbasierte Richtlinien und Empfehlungen für die Diagnostik und Therapie kryptoglandulärer Analfisteln zusammengestellt.

\section{Schlüsselwörter}

Analfistel · Stuhlinkontinenz - Operative Therapie · Diagnostik · Literaturrecherche

\section{S3 guidelines: cryptoglandular anal fistulas. 2. revised version, The German Association of Scientific Medical Societies registration number: 088/003}

\section{Abstract}

Background. Fistula-in-ano of cryptoglandular origin is a common disease with an incidence of 2 per 10,000 inhabitants per year and the peak incidence is in young male adults. Inadequate treatment can have adverse effects on the quality of life and in particular lead to a reduction of fecal continence.

Methods. A new systematic review of the literature was performed based on the $\mathrm{S} 3$ guidelines published in 2011 in order to develop updated guidelines for anal fistulas. Results. Relevant randomized studies are rare and the level of evidence is generally low. The classification and diagnostics of anal fistulas are still based on the unchanged recommendations from the previous publication. The therapy of anal fistulas can be performed by one of the following operative procedures: fistulotomy or lay-open technique, drainage seton, plastic surgery reconstruction techniques including suturing of the sphincter or occlusion with biomaterials. The new ligation of the intersphincteric fistula tract (LIFT) technique was presented in several studies. The results with respect to healing rates and continence disorders largely correspond to those of plastic reconstruction techniques. The assessment of other new techniques, such as laser, video-assisted anal fistula treatment (VAAFT) and over the scope (OTS) clips, is currently not possible due to a lack of representative literature. The lay-open technique should only be performed in cases of superficial fistulas. The risk of postoperative incontinence increases with the amount of sphincter muscle incised. In cases of high anal fistulas a sphincter preserving procedure should be preferred. The results of the various reconstruction techniques are relatively similar. Using biomaterials for occlusion the healing rate is much lower than originally reported. An assessment of the importance of new materials, such as plugs, collagen and autologous stem cells, is also not possible due to a lack of evidence.

Conclusion. These revised $\mathrm{S} 3$ guidelines provide evidence-based protocols and recommendations for the diagnostics and treatment options of cryptoglandular fistulain-ano based on the currently available literature.

\section{Keywords}

Fistula-in-ano · Fecal incontinence - Operative treatment · Diagnostic procedure - Literature review wies keine Interessenkonflikte in Bezug auf Firmen, die Produkte zur Behandlung von Analfisteln vertreiben, auf. Der Text wurde Punkt für Punkt durchgesprochen und diskutiert. Der Text war allen Mitgliedern zuvor per Mail zugegangen. Unklare Stellen wurden nach Diskussion korrigiert. Die Abstimmung er- folgte per Handzeichen. Alle anwesenden Mitglieder der Leitliniengruppe hatten die gleiche Stimmberechtigung. Die Mitglieder der Leitliniengruppe, die an den Treffen nicht teilnehmen konnten, stimmten dem Text später getrennt zu. Auch die jeweiligen Vorstände der beteiligten Fachgesellschaften bekundeten ihre Zustimmung zu der vorliegenden definitiven Textfassung. Eine methodische Überprüfung des Textes erfolgte durch Frau Muche-Borowski als Vertreterin der AWMF. Eine kritische Beurteilung nach dem DELBI-Verfahren fand durch die beiden Chirurgen Dr. Roland Scherer, 


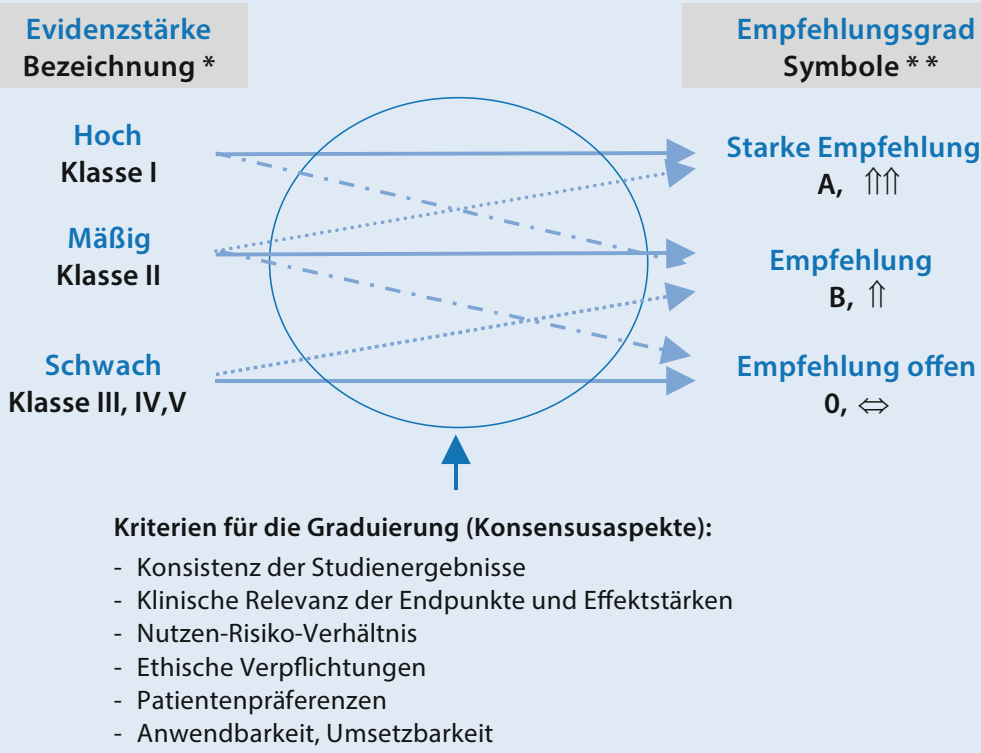

Empfehlungsgrad Symbole **

Starke Empfehlung A, $\Uparrow \Uparrow$

Empfehlung

B, 介

Empfehlung offen $0, \Leftrightarrow$

Kriterien für die Graduierung (Konsensusaspekte):

- Konsistenz der Studienergebnisse

- Klinische Relevanz der Endpunkte und Effektstärken

- Nutzen-Risiko-Verhältnis

- Ethische Verpflichtungen

- Patientenpräferenzen

- Anwendbarkeit, Umsetzbarkeit

Abb. 1 A Korrelation zwischen Evidenzstärke (Evidenzgrad) und Empfehlungsgrad. (Mod. nach [323, 367])

Berlin, und Dr. Gerd Kolbert, Hannover, statt.

Eine Liste möglicher Interessenkonflikte aller an der Erstellung dieser Leitlinie beteiligten Ärzte ist online unter http://www.awmf.org/leitlinien/detail/ 11/088-003.html nachzulesen. Keiner der Autoren gibt Interessenskonflikte an, die in direktem Zusammenhang mit den Aussagen dieser Leitlinien stehen.

Die nächste Überarbeitung der Leitlinie wird angestrebt für 03/2021.

\section{Evidenztabellen}

Im Rahmen der Aufarbeitung der vorhandenen Literatur zur operativen Behandlung von Analfisteln wurden Tabellen (• Tab. 4-22) zusammengestellt, die die Ergebnisse der Literatur in Bezug auf die einzelnen Operationsverfahren darstellen. Dabei wurden folgende Ergebnisse der einzelnen Studien erfasst:

- Anzahl der behandelten Patienten,

- Typ der behandelten Fisteln,

- Anteil von Patienten mit M. Crohn

(Studien mit einem Anteil von mehr als $20 \%$ wurden nicht ausgewertet),

- Studiendesign (retro-/prospektiv, randomisiert),
- Heilungsrate (definiert als vollständiger Verschluss der äußeren Wunde nach der Erstoperation),

- Rezidivrate (definiert als Wiederauftreten der Fistel nach vorhergehender kompletter Abheilung),

- Häufigkeit von Kontinenzstörungen,

- Follow-up-Zeit.

\section{Historischer Hintergrund}

Analabszesse und ihre Therapie mittels Abszessinzision, Fadendrainage und Fistelspaltung wurden bereits $450 \mathrm{v}$. Chr. von Hippokrates beschrieben. John von Ardene führte 1350 n. Chr. eine Sondierung von Haupt- und Nebengängen durch [41]. Im Jahr 1602 findet eine Analfistel als Erkrankung Eingang in die Literatur in Shakespeares Schauspiel „All's well that ends well“ [87] und im Jahr 1687 wurde durch Felix eine Analfisteloperation bei Ludwig XIV. von Frankreich vorgenommen. In den Jahren 1902 und 1912 stellten Noble [285] und Elting [124] schließmuskelschonende Operationstechniken vor. Die erste umfangreiche wissenschaftliche Aufarbeitung erfolgte durch Stelzner 1959 in „Die anorektalen Fisteln“ [409].

\section{Epidemiologie}

Analabszesse und Fisteln finden sich überwiegend bei jüngeren Erwachsenen zwischen dem 30. und 50. Lebensjahr [258, 288, 341, 473], wobei Männer deutlich häufiger als Frauen betroffen sind.

Im Jahr 1984 beschrieben Saino et al. [358] die Epidemiologie der Analfistel anhand der Bevölkerung Helsinkis (510.000 Einwohner). Die Inzidenz lag bei 8,6 pro 100.000 Einwohner und war bei Männern deutlich höher als bei Frauen (12,3 vs. 5,6). Von den im Beobachtungszeitraum von 1969 bis 1978 diagnostizierten 458 Fisteln waren $90 \%$ kryptoglandulärer Genese. Das mittlere Alter bei der ersten Manifestation betrug 38,3 Jahre.

Zanotti et al. [490] untersuchten im Jahr 2007 die Inzidenz in vier europäischen Ländern anhand von Patientendatenbanken. Die niedrigste Inzidenz wurde in Spanien mit 1,04 pro 10.000 Einwohner/Jahr und die höchste in Italien mit 2,32 pro 10.000 Einwohner/Jahr ermittelt. Die Inzidenz in England betrug 1,84 pro 10.000 Einwohner/Jahr und in Deutschland 2,02 pro 10.000 Einwohner/ Jahr. Relevante neue Arbeiten zu diesem Thema sind seit 2011 nicht erschienen.

Im Geschlechtervergleich zeigten Mitalas et al. [272] eine Häufung von anterioren tiefen transsphinktären Analfisteln bei Frauen. Diese manifestierten sich seltener primär als Abszess.

Eine spezielle Fistelform z. T. kongenitaler Genese findet sich bei Kindern im 1. Lebensjahr. Kryptoglanduläre Fisteln bei älteren Kindern sind sehr selten und oft mit einer chronisch-entzündlichen Darmerkrankung assoziiert. Sieverlaufen meistens oberflächlich und können durch Spaltung behandelt werden [284, 385]. Die vorliegende Leitlinie ist somit nur bedingt auf Kinder übertragbar.

Fazit für die Praxis. Das Fistelleiden ist eine Erkrankung, welche überwiegend beijungen männlichen Erwachsenen auftritt. Die Inzidenz in verschiedenen Populationen variiert. 
Tab. 3 Mitglieder der Konsensusgruppe

Für die Deutsche Gesellschaft für Allgemein- und Viszeralchirurgie (DGAV), die Chirurgische Arbeitsgemeinschaft für Coloproktologie (CACP), die Deutsche Gesellschaft für Koloproktologie (DGK), den Berufsverband der Coloproktologen Deutschlands (BCD)

Dr. A. Ommer, End- und Dickdarmdarm-Zentrum, Essen

Prof. Dr. A. Herold, Enddarmzentrum, Mannheim

Dr. E. Berg, Prosper Hospital, Recklinghausen,

Prof. Dr. St. Farke, Klinikum Delmenhorst, Delmenhorst

Prof. Dr. A. Fürst, Caritas Krankenhaus, Regensburg,

Prof. Dr. F. Hetzer, Spital Linth, Uznach, Schweiz

Dr. A. Köhler, HELIOS St. Johannes Klinik Duisburg, Duisburg

Prof. Dr. S. Post, Universitätsklinik Mannheim, Mannheim

Dr. R. Ruppert, Städt. Klinikum München GmbH, Klinikum Neuperlach, München,

Prof. Dr. M. Sailer, Bethesda-Krankenhaus, Hamburg

Prof. Dr. Th. Schiedeck, Klinikum Ludwigsburg, Ludwigsburg

Prof. Dr. O. Schwandner, Krankenhaus Barmherzige Brüder, Regensburg

Dr. B. Strittmatter, Praxisklinik 2000, Praxis für Koloproktologie, Freiburg

Für die Deutsche Dermatologische Gesellschaft (DDG)

Dr. B. H. Lenhard, Praxis für Enddarmerkrankungen, Heidelberg

Für die AGUB der Deutschen Gesellschaft für Gynäkologie und Geburtshilfe

Prof. Dr. W. Bader, Klinikum Bielefeld, Bielefeld

Für die Deutsche Gesellschaft für Urologie (DGU)

Prof. Dr. S. Krege, Kliniken Essen-Mitte, Essen

Für die Deutsche Gesellschaft für Gastroenterologie, Verdauungs- und Stoffwechselkrankheiten (DGVS)

Prof. Dr. H. Krammer, Gastroenterologie am End-Darm-Zentrum, Mannheim

Prof. Dr. E. Stange, Robert-Bosch-Krankenhaus, Stuttgart

\section{Ätiologie und Definition}

Ausgangspunkt des entzündlichen Geschehens sind die beim Menschen nur rudimentär angelegten Proktodealdrüsen im Intersphinktärraum, die erstmals 1880 von Herman und Defosses und 1967 von Conole [84] beschrieben wurden [175]. Sie besitzen einen Ausführungsgang, der im Analkanal auf Höhe der Linea dentata mündet. Im Organismus können purulente Keime eine umschriebene akute Entzündung mit Ausbildung von Nekrosen verursachen. Diese Nekrosen werden von einem Granulationsgewebe, der sog. Abszesskapsel, umgeben. Je nach Virulenz der Keime breitet sich der Abszess in Richtung des geringsten Widerstands aus und erreicht auf diese Weise die benachbarte Oberfläche. Die entstandene Verbindung des in der Tiefe gelegenen und mit Eiter gefüllten Raums mit der Körperoberfläche wird mit Granulationsgewebe ausgekleidet. Dieser Kanal wird als Fistel bezeichnet.
Wird der unnatürliche Verbindungsgang gegen die Umgebung einseitig abgedichtet, entsteht eine Ventilfistel mit Sekretstau. Das akute entzündliche Stadium wird als Analabszess und der chronische Verlauf mit putrider Sekretion bei vorhandener äußerer Öffnung als Analfistel bezeichnet. Mehrere Studien beschreiben eine individuelle Varianz der Anzahl der Proktodealdrüsen und ihrer Ausführungsgänge mit einer posterioren Häufung [383]. Die Zahl der Drüsen ist bei Männern höher als bei Frauen [235]. Die Ausbreitung des Infekts aus dem intersphinktären Raum bestimmt den späteren Fistelverlauf [115, 245, 426].

Eine niederländische Arbeitsgruppe untersuchte die Epithelialisation in Analfisteln [451]. Bei tiefen Analfisteln fanden sie Epithelauskleidung im Fisteltrakt und verweisen als Folgerung auf die Notwendigkeit einer Fistelkürettage im Rahmen der Operation zur Verbesserung des Heilungserfolgs. In einer Folgearbeit [272] wurde eine histologische Untersuchung

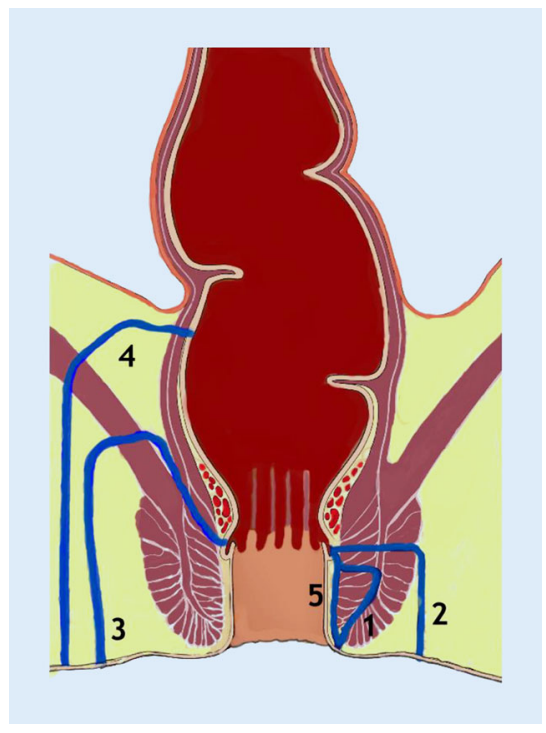

Abb. 2 A Klassifikation der Analfisteln. 1 intersphinktär, 2 transsphinktär, 3 suprasphinktär, 4 extrasphinktär, 5 subanodermal. (Mod. nach [200] und [313])

des Fisteltrakts nach LIFT-Operation durchgeführt. Hier fand sich bei der transsphinktären Analfistel nur bei $25 \%$ der Resektate eine Epithelialisation, so dass diese keine signifikante Rolle zu spielen scheint.

Die mikrobiologische Untersuchung weist i. d. R. eine Mischflora auf und ist für die Behandlung der kryptoglandulären Analfisteln nicht relevant [243, 382]. Dies wurde auch in einer aktuellen Studie [437] bestätigt. Andere Keime, z. B. Tuberkelbakterien, sind ausgesprochen selten [33, 137].

In neueren Publikationen wurden weitere pathogenetische Faktoren für eine Fistelbildung herausgearbeitet, wie Lebensstil, Adipositas, hoher täglicher Salzkonsum, Diabetes mellitus, Hyperlipidämie, Vorhandensein von Dermatosen, Rauchen, Alkoholkonsum, sitzende Tätigkeit, exzessiv scharfe Speisen, mangelnde Bewegung und längeres Sitzen auf der Toilette bei der Defäkation [471] sowie psychosozialer Stress [81]. Zu ähnlichen Ergebnissen kommt auch eine schwedische Studie [13]: Neben dem Morbus Crohn erhöht die Prävalenz von Diabetes und Adipositas das Risiko für die Entstehung eines Analabszesses.

Fazit für die Praxis. Das Analfistelleiden entsteht vorwiegend aus einer akuten 


\begin{tabular}{|c|c|c|c|c|c|c|c|c|c|}
\hline Autor & Jahr & $\begin{array}{l}\text { Anzahl } \\
\text { (n) }\end{array}$ & Fisteltyp & $\begin{array}{l}\text { Anteil } \\
\text { M. Crohn } \\
(\%)\end{array}$ & Studiendesign & Heilung (\%) & Rezidiv (\%) & $\begin{array}{l}\text { Kontinenz- } \\
\text { störung (\%) }\end{array}$ & $\begin{array}{l}\text { Follow-up } \\
\text { (Monate) }\end{array}$ \\
\hline Stelzner [411] & 1956 & 73 & II & k. A. & Retrospektiv & 100 & k. A. & $\begin{array}{l}\text { Grad 3: } 10 \\
\text { "Schwäche": } \\
30\end{array}$ & $60-120$ \\
\hline Kügler [222] & 1966 & 54 & $\|$ & k. A. & Retrospektiv & 100 & k. A. & 33 & k. A. \\
\hline Akvobianz [17] & 1968 & 40 & I, II, III & k. A. & Retrospektiv & 100 & k. A. & 20 & $24-48$ \\
\hline Parks [314] & 1976 & $\begin{array}{l}9 \\
6\end{array}$ & $\begin{array}{l}\text { II } \\
\text { III }\end{array}$ & k. A. & $\begin{array}{l}\text { Retrospektiv } \\
\text { VG: - Tab. } 5\end{array}$ & $\begin{array}{l}78 \\
50\end{array}$ & k. A. & k. A. & $>12$ \\
\hline Riedler [343] & 1978 & 107 & I, II, III & k. A. & Retrospektiv & $74 ?$ & k. A. & 21 & k. A. \\
\hline Saino [359] & 1985 & 199 & I, II, III & k. A. & Retrospektiv & $\begin{array}{l}89 \\
\text { „Hohe" Fistel: } \\
74\end{array}$ & k. A. & 34 & $7-108$ \\
\hline Vasilewsky [462] & 1985 & 160 & II, III & k. A. & Retrospektiv & 94 & k. A. & 0 & k. A. \\
\hline Shouler [389] & 1986 & 96 & 1 & k. A. & Retrospektiv & 94 & 0 & 13 & $2,5(1-50)$ \\
\hline Kennedy ${ }^{a}$ [207] & 1990 & 32 & $\begin{array}{l}\text { II (distal), } \\
\text { III }\end{array}$ & 0 & $\begin{array}{l}\text { Retrospektiv } \\
\text { VG: } \bullet \text { Tab. } 5\end{array}$ & 78 & k. A. & $\begin{array}{l}\text { Grad 1: } 44 \\
\text { Grad 2: } 3\end{array}$ & $36(13-36)$ \\
\hline Sangwan [361] & 1994 & 461 & I, lla & 0 & Retrospektiv & k. A. & 6,5 & k. A. & 34 \\
\hline Van Tets [458] & 1994 & 312 & I-IV & 0 & $\begin{array}{l}\text { Retrospektiv } \\
\text { VG: Tab. } 7\end{array}$ & 100 & 6 & Minor: 27 & 12 \\
\hline $\begin{array}{l}\text { Garcia-Aguilar } \\
\text { [134] }\end{array}$ & 1996 & 108 & $\begin{array}{l}\text { I, II, III, IV, } \\
\text { unklassifi- } \\
\text { ziert }\end{array}$ & 0 & $\begin{array}{l}\text { Retrospektiv } \\
\text { VG: • Tab. } 8\end{array}$ & 92 & k. A. & $\begin{array}{l}45 \\
\text { Typ I: } 37 \\
\text { Typ II: } 54 \\
\text { Typ III } 80\end{array}$ & 29 \\
\hline Hongo $^{b}$ [187] & 1997 & 319 & $\begin{array}{l}\text { I, Il (proxi- } \\
\text { mal) }\end{array}$ & k. A. & Retrospektiv & 85 & 1,5 & k. A. & k. A. \\
\hline Iwadare $^{b}$ [194] & 1997 & 328 & $\|$ & k. A. & Retrospektiv & 98,5 & k. A. & k. A. & k. A. \\
\hline Ho [183] & 1998 & $\begin{array}{l}51(A) \\
51(B)\end{array}$ & I, II & 0 & Randomisiert ${ }^{c}$ & $\begin{array}{l}96 \\
98\end{array}$ & k. A. & $\begin{array}{l}2 \\
12\end{array}$ & $\begin{array}{l}9 \\
10,2\end{array}$ \\
\hline Ho [182] & 2001 & 54 & $\|$ & 0 & $\begin{array}{l}\text { Randomisiert }^{\mathrm{d}} \\
\text { VG: - Tab. } 7\end{array}$ & 100 & k. A. & 5,6 & $\begin{array}{l}1,9 \\
(0,4-12,3)\end{array}$ \\
\hline Mylonakis [279] & 2001 & 65 & I, II & 0 & $\begin{array}{l}\text { Retrospektiv } \\
\text { VG: Tab. } 7\end{array}$ & 98,5 & k. A. & Minor: 38,5 & 3 \\
\hline Lindsey [238] & 2002 & 7 & $\begin{array}{l}\text { I, II (proxi- } \\
\text { mal) }\end{array}$ & Ja & $\begin{array}{l}\text { Randomisiert }{ }^{\mathrm{e}} \\
\text { VG: } \bullet \text { Tab. } 14\end{array}$ & 100 & k. A. & 0 & 3 \\
\hline Cavanaugh $^{f}[59]$ & 2002 & 93 & I, II & 0 & Retrospektiv & 100 & k. A. & $\begin{array}{l}\text { Lebensquali- } \\
\text { tätsreduktion } \\
\text { leicht: } 14 \text {, } \\
\text { deutlich: } 10 \\
\text { Vorlagen: } 8 \\
64 \text { (Score) }\end{array}$ & $<24$ bis $>60$ \\
\hline Westerterp ${ }^{9}[479]$ & 2003 & 46 & I, II & 0 & Retrospektiv & 100 & 0 & $\begin{array}{l}A: 24 \\
B: 44 \\
C: 82\end{array}$ & $12-48$ \\
\hline Chang [64] & 2003 & 45 & I, lla & 0 & Retrospektiv & 93 & k. A. & $\begin{array}{l}\text { Vor allem Frau- } \\
\text { en, Patienten } \\
\text { mit niedrigem } \\
\text { Ruhedruck }\end{array}$ & $9,5 \pm 3,2$ \\
\hline Pescatori [322] & 2006 & $\begin{array}{l}22(A) \\
24(B)\end{array}$ & $\begin{array}{l}\text { I-IV, Rezi- } \\
\text { div }\end{array}$ & 0 & Randomsiert $^{\mathrm{h}}$ & $\begin{array}{l}86 \\
79\end{array}$ & k. A. & k. A. & $10,5 \pm 13,8$ \\
\hline $\begin{array}{l}\text { Van der } \\
\text { Hagen' [445] }\end{array}$ & 2006 & 62 & $\begin{array}{l}\text { I, II (proxi- } \\
\text { mal) }\end{array}$ & $15(9 / 62)$ & Retrospektiv & 98 & $\begin{array}{l}7 \\
16 \\
26 \\
39\end{array}$ & 4,8 & $\begin{array}{l}12 \\
24 \\
48 \\
72\end{array}$ \\
\hline
\end{tabular}




\begin{tabular}{|c|c|c|c|c|c|c|c|c|c|}
\hline Autor & Jahr & $\begin{array}{l}\text { Anzahl } \\
(n)\end{array}$ & Fisteltyp & $\begin{array}{l}\text { Anteil } \\
\text { M. Crohn } \\
(\%)\end{array}$ & Studiendesign & Heilung (\%) & Rezidiv (\%) & $\begin{array}{l}\text { Kontinenz- } \\
\text { störung (\%) }\end{array}$ & $\begin{array}{l}\text { Follow-up } \\
\text { (Monate) }\end{array}$ \\
\hline $\begin{array}{l}\text { Van Koperen } \\
\text { [452] }\end{array}$ & 2008 & 109 & $\begin{array}{l}\text { I, II (proxi- } \\
\text { mal) }\end{array}$ & 0 & $\begin{array}{l}\text { Retrospektiv } \\
\text { VG: } \bullet \text { Tab. } 9 \\
\text { und } 10\end{array}$ & k. A. & 7 & 40 & $76(7-134)$ \\
\hline 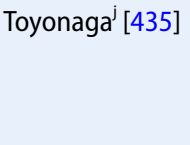 & 2007 & $\begin{array}{l}35(\mathrm{~A}) \\
35(\mathrm{~B})\end{array}$ & $\begin{array}{l}\text { II (distal), } \\
\text { III }\end{array}$ & 0 & Prospektiv & $\begin{array}{l}97 \\
94\end{array}$ & k. A. & $\begin{array}{l}51 \text { (minor: } 49, \\
\text { major: } 3 \text { ) } \\
28 \text { (minor: } 25, \\
\text { major: } 3 \text { ) }\end{array}$ & $12(6-20)$ \\
\hline Jordan [202] & 2008 & 132 & I-IV & 0 & $\begin{array}{l}\text { Retrospektiv } \\
\text { VG: } \bullet \text { Tab. } 9 \\
\text { und } 12\end{array}$ & 98,5 & k. A. & k. A. & $19 \pm 25$ \\
\hline Bokhari [46] & 2009 & $\begin{array}{l}57 \\
38\end{array}$ & $\begin{array}{l}\text { Einfach } \\
\text { Komplex }\end{array}$ & 0 & Retrospektiv & $\begin{array}{l}93 \\
79\end{array}$ & k. A. & $\begin{array}{l}16 \\
37\end{array}$ & k. A. \\
\hline Ortiz [299] & 2008 & 115 & $\begin{array}{l}\text { I, II (proxi- } \\
\text { mal) }\end{array}$ & k. A. & $\begin{array}{l}\text { Retrospektiv } \\
\text { VG: • Tab. } 10\end{array}$ & k. A. & 2 & 6,1 & 42 \\
\hline Atkin $[28,29]$ & 2011 & $\begin{array}{l}52 \\
84\end{array}$ & $\begin{array}{l}\text { I } \\
\text { II }\end{array}$ & 0 & Retrospektiv & $\begin{array}{l}98 \\
96\end{array}$ & $\begin{array}{l}0 \\
2(2 / 96)\end{array}$ & $\begin{array}{l}\text { Typ I: } \\
\text { Grad 1: 18, } \\
\text { Grad 2: 2, } \\
\text { Grad 3: } 0 \\
\text { Typ II: } \\
\text { Grad 1: 23, } \\
\text { Grad 2: 4, } \\
\text { Grad 3: } 2\end{array}$ & $5(1-114)$ \\
\hline Jain [196] & 2012 & $\begin{array}{l}40 \\
\text { A: } 20 \\
\text { Fistelek- } \\
\text { tomie } \\
\text { B: } 20 \\
\text { Spal- } \\
\text { tung mit } \\
\text { Marsupa- } \\
\text { lisation }\end{array}$ & I, II („low“) & 0 & Randomisiert & $\begin{array}{l}100 \\
\text { Heilungsdauer: } \\
\text { A: } 5 \pm 1, B: 7 \pm \\
2\end{array}$ & k. A. & 0 & 3 \\
\hline Tozer $^{k}$ [438] & 2013 & 50 & I, II & 10 & Retrospektiv & 90 & k. A. & $\begin{array}{l}\text { Minor: } 18 \\
\text { Major: } 8\end{array}$ & k. A. \\
\hline Hall [165] & 2014 & 146 & I, II, RVF & 12 & $\begin{array}{l}\text { Prospektiv, } \\
\text { multizentrisch } \\
\text { (16 Operateu- } \\
\text { re) } \\
\text { VG: } \\
\text { - Tab. 9, } 14 \\
\text { und } 17\end{array}$ & 94 & k. A. & $\begin{array}{l}\text { CCFI postop. } \\
\text { niedriger }\end{array}$ & 3 \\
\hline
\end{tabular}

Entzündung der Proktodealdrüsen im Intersphinktärraum. Die Ausbreitung der Infektion bestimmt den späteren Fistelverlauf. Lebensstil und psychosoziale Faktoren könnten das Risiko für eine Fistelbildung erhöhen.

\section{Klassifikation}

In der klinischen Routine hat sich die Einteilung der Analfisteln nach ihrer Beziehung zum Analsphinkter bewährt. Die ICD-Klassifikation ist zur Einteilung nicht geeignet, da lediglich ein
Code (ICD K 60.3) für alle Analfisteln zur Verfügung steht.

Häufig eingesetzt wird die Einteilung nach Parks, wobei die Originalbeschreibung mit Berücksichtigung von Nebengängen und nichtdrainierten Granulationshöhlen oft für die tägliche Routine zu umfassend ist [313]. Wir empfehlen die in $\bullet$ Abb. 2 dargestellte Klassifikation, die sich nur an den Hauptgruppen orientiert. Die Verbindung zum Analkanal erfolgt entweder durch eine Persistenz des Drüsenausführungsgangs oder eine traumatische Öffnung im Rahmen einer
Spontanperforation oder chirurgisch induziert.

Ausgehend von der Kryptendrüse mit ihrem Ausführungsgang auf Höhe der Linea dentata verlaufen die intersphinktären Fisteln (Typ I nach Parks) im intersphinktären Spalt nach distal und münden neben dem Analkanal in die perianale Haut. In einigen Fällen können intersphinktäre Fisteln auch oberflächliche distale Anteile des M. sphincter ani externus mit umschließen. Die transsphinktären Fisteln (Typ II nach Parks) durchbohren den M. sphincter ani externus in die Fossa ischiorectalis und 


\begin{tabular}{|c|c|c|c|c|c|c|c|c|c|}
\hline Autor & Jahr & $\begin{array}{l}\text { Anzahl } \\
\text { (n) }\end{array}$ & Fisteltyp & $\begin{array}{l}\text { Anteil } \\
\text { M. Crohn } \\
(\%)\end{array}$ & Studiendesign & Heilung (\%) & Rezidiv (\%) & $\begin{array}{l}\text { Kontinenz- } \\
\text { störung (\%) }\end{array}$ & $\begin{array}{l}\text { Follow-up } \\
\text { (Monate) }\end{array}$ \\
\hline Göttgens' [150] & 2015 & 537 & I, II, V & 7 & $\begin{array}{l}\text { Retrospektiv, } \\
\text { multizentrisch }\end{array}$ & $\begin{array}{l}83,6 \text { (nach } \\
\text { mehrfacher } \\
\text { Op.: 90,3\%) }\end{array}$ & k. A. & $\begin{array}{l}\text { Keine: } 26 \\
\text { Major: } 28 \\
\text { Lebensqualität } \\
\text { entspricht } \\
\text { Allgemeinbe- } \\
\text { völkerung }\end{array}$ & $>60$ \\
\hline Dutta [110] & 2015 & 24 & $\begin{array}{l}\text { I (74\%), II } \\
(26 \%)\end{array}$ & k. A. & $\begin{array}{l}\text { Randomisiert } \\
\text { VG: } \\
\text { V Tab. } 7\end{array}$ & $\begin{array}{l}100 \text { (Heilungs- } \\
\text { dauer: } 36 \\
\text { Tage) }\end{array}$ & k. A. & 12 & k. A. \\
\hline \multicolumn{10}{|c|}{ 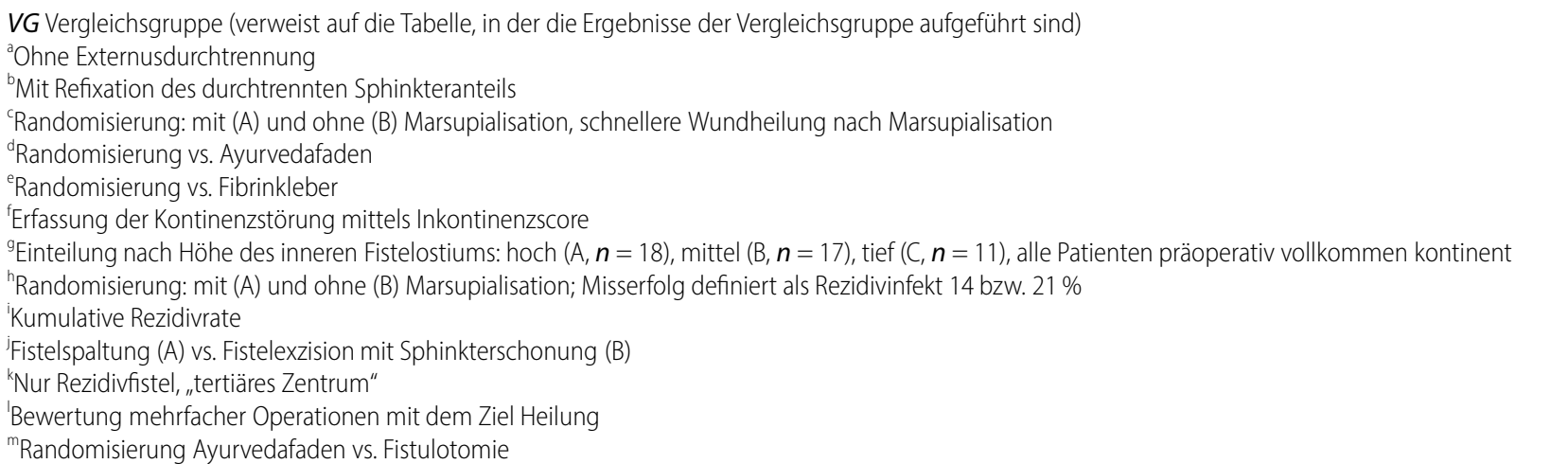 } \\
\hline
\end{tabular}

münden in die Haut perianal. Im klinischen Alltag ist es sinnvoll, zwischen distalen und proximalen transsphinktären Fisteln in Abhängigkeit vom Ausmaß des betroffenen Schließmuskelanteils zu unterscheiden. Eine Sonderform stellt die Hufeisenfistel dar, deren innere Öffnung bei 6.00 oder 12.00 Uhr in Steinschnittlage (SSL) liegt und die äußere Öffnungen auf beiden Glutealseiten aufweist. Insgesamt ist die Unterscheidung zwischen inter- und transsphinktärem Verlauf nicht immer problemlos. Suprasphinktäre Fisteln (Typ III nach Parks) verlaufen zunächst im intersphinktären Raum nach proximal kranial in die Fossa pelvirectalis, durchbohren dann den M. levator ani und verlaufen durch die Fossa ischiorectalis zur perianalen Haut. Diese letztere Verbindung wird vermutlich begünstigt durch intramuskuläre Spalten („gaps“) im Levator, oder sie entstehen durch die operative translevatorische Entlastung eines Abszesses.

Die extrasphinktären Fisteln (Typ IV nach Parks) sind i. d. R. nicht kryptoglandulären Ursprungs, d.h. ihre Mündung liegt nicht auf Höhe der Linea dentata. Ihre Beziehungen zum Sphinkter sind unterschiedlich. Auch ein direkter Verlauf vom Abdomen durch das Mesorektum, den M. levator ani und die Fossa ischiorectalis ist möglich [312]. Aus diesem Grund können extrasphinktäre Fisteln nur anhand des individuellen Einzelfalls beschrieben werden. Sie sind abhängig von der jeweiligen Genese (z. B. Divertikulitis, M. Crohn, iatrogen), stellen somit keine typischen Analfisteln dar und sind nicht Thema der vorliegenden Leitlinie.

Von einigen Autoren werden auch subkutane bzw. subanodermale Fisteln beschrieben [253], die ebenfalls keine direkte Beziehung zum Schließmuskelapparat aufweisen. Diese sind häufig im Zusammenhang mit Fissuren zu finden.

Die Häufigkeitsverteilung der Fisteltypen variiert in der Literatur [24, 217, 249, $253,313,348,462]$. So liegt die Häufigkeit der intersphinktären Fistel zwischen 24 und $54 \%$, der transsphinktären zwischen 30 und $58 \%$ sowie der suprasphinktären zwischen 1 und $20 \%$. Die Häufigkeit der extrasphinktären Fisteln wird mit bis zu $5 \%$ in älteren Publikationen [313] und bis zu $3 \%$ in neueren Publikationen [349] angegeben. Zu berücksichtigen ist jedoch die Tatsache, dass alle Zahlen aus dem Krankengut koloproktologischer Spezialabteilungen stammen, denen insbeson- dere komplexe Fisteln (Typ II, III, IV, rektovaginal) zugewiesen werden, so dass hier eine Verschiebung der Zahlen zugunsten dieser Typen anzunehmen ist, wie es das von Malouf et al. aufgearbeitete Patientengut des St. Mark's Hospital impliziert [249].

Einen wichtigen Stellenwert bei der Diagnostik kommt der sog. Goodsall-Regel zu [491]. Diese besagt, dass Fisteln, deren äußere Öffnung in Steinschnittlage unterhalb einer horizontalen Linie, die durch Anus und beide Sitzbeinhöcker verläuft, meist bogenförmig verlaufen und im Analkanal bei 6.00 SSL münden, während Fisteln, die sich oberhalb dieser Linie perianal öffnen, meist geradlinig auf den Analkanal zuziehen. Eine umfangreiche Aufarbeitung von Cirocco und Reilly [83] bestätigte diese Regel v. a. für posteriore Fisteln, während dies für die anterioren Fisteln nur in der Hälfte der Fälle zutraf. Unterstützt werden diese Daten von weiteren Publikationen $[35,85,148]$, die andererseits auch darauf hinweisen, dass diese Regel für CrohnFisteln meistens nicht zutrifft. Eine aktuelle Studie [7] untersucht den Zusammenhang zwischen der Verteilung der Proktodealdrüsen und der Fistellokali- 
Tab. 5 Ergebnisse der Fadenmethode (Faden als Drainage („loose seton“)

\begin{tabular}{|c|c|c|c|c|c|c|c|c|c|}
\hline Autor & Jahr & $\begin{array}{l}\text { Anzahl } \\
(n)\end{array}$ & Fisteltyp & $\begin{array}{l}\text { Anteil } \\
\text { M. Crohn } \\
(\%)\end{array}$ & Studiendesign & $\begin{array}{l}\text { Heilung } \\
(\%)\end{array}$ & Rezidiv (\%) & $\begin{array}{l}\text { Kontinenz- } \\
\text { störung (\%) }\end{array}$ & $\begin{array}{l}\text { Follow-up } \\
\text { (Monate) }\end{array}$ \\
\hline Parks ${ }^{\mathrm{a}}[314]$ & 1976 & $\begin{array}{l}23 \\
57\end{array}$ & $\begin{array}{l}\text { II } \\
\text { III }\end{array}$ & k. A. & $\begin{array}{l}\text { Retrospektiv } \\
\text { VG: Tab. } 4\end{array}$ & $\begin{array}{l}81 \\
60\end{array}$ & k. A. & $\begin{array}{l}17 \\
39\end{array}$ & $>12$ \\
\hline Brühl ${ }^{b}[48]$ & 1986 & 704 & I, II & k. A. & Retrospektiv & 64 & 3,5 & k. A. & k. A. \\
\hline Thompson ${ }^{\mathrm{a}}$ [427] & 1989 & 34 & $\|$ & 0 & $\begin{array}{l}\text { Retrospektiv } \\
\text { VG: } \bullet \text { Tab. } 6\end{array}$ & 56 & k. A. & 17 & $55(20-159)$ \\
\hline Kennedy ${ }^{\mathrm{a}}$ [207] & 1990 & 32 & II, III & 0 & $\begin{array}{l}\text { Retrospektiv } \\
\text { VG: Tab. } 4\end{array}$ & 78 & k. A. & 62 & $39(21-90)$ \\
\hline Williams ${ }^{\mathrm{a}}$ [484] & 1991 & 14 & $\begin{array}{l}\text { II (distal), } \\
\text { III }\end{array}$ & 0 & $\begin{array}{l}\text { Retrospektiv } \\
\text { VG: } \bullet \text { Tab. } 6 \\
\text { und } 7\end{array}$ & 86 & k. A. & 43 & $24(4-60)$ \\
\hline Williams ${ }^{\mathrm{a}}$ [484] & 1991 & 23 & Ilb, III & 100 & Retrospektiv & 61 & k. A. & 26 & $24(4-60)$ \\
\hline $\operatorname{Graf}^{c}[154]$ & 1995 & 29 & ॥ & $14(4 / 29)$ & Retrospektiv & 92 & k. A. & 52 & 46 \\
\hline Garcia-Aguilar ${ }^{\mathrm{a}}$ [134] & 1996 & 51 & II, III & k. A. & $\begin{array}{l}\text { Retrospektiv, } \\
\text { VG: • Tab. } 4\end{array}$ & 90 & k. A. & 63 & k. A. \\
\hline Lentner $^{\mathrm{a}}[233]$ & 1996 & 79 & $\begin{array}{l}\text { I, II (proxi- } \\
\text { mal) }\end{array}$ & k. A. & Retrospektiv & 92,3 & k. A. & 0,9 & 16 \\
\hline Mohite $^{d}[274]$ & 1997 & 114 & k. A. & k. A. & Retrospektiv & 100 & $\begin{array}{l}0 \\
\text { (4-mal Ab- } \\
\text { szessrezidiv) }\end{array}$ & 0 & $6-30$ \\
\hline Garcia-Aguilar [133] & 1998 & 47 & $\begin{array}{l}\text { II (distal), } \\
\text { III, IV }\end{array}$ & k. A. & $\begin{array}{l}\text { Retrospektiv } \\
\text { VG: } \bullet \text { Tab. } 7\end{array}$ & 91 & k. A. & 66 & 33 \\
\hline Joy $^{a}[203]$ & 2002 & 12 & II, III & $5(3 / 63)$ & $\begin{array}{l}\text { Retrospektiv } \\
\text { VG: } \bullet \text { Tab. } 7 \\
\text { und } 10\end{array}$ & 75 & k. A. & 50 & $19(9-54)$ \\
\hline Chung [77] & 2006 & 86 & II, III & 0 & $\begin{array}{l}\text { Prospektiv } \\
\text { VG: } \\
\text { - Tab. } 9,14 \\
\text { und } 17\end{array}$ & 33 & k. A. & k. A. & 3 \\
\hline Eitan [116] & 2009 & 41 & $\|$ & $7(3 / 41)$ & Retrospektiv & 80,5 & k. A. & 15 & $<12$ \\
\hline Choi ${ }^{9}[69]$ & 2010 & $\begin{array}{l}12 \\
12\end{array}$ & $\begin{array}{l}\text { II, III (Huf- } \\
\text { eisenfistel) }\end{array}$ & 0 & Retrospektiv & $\begin{array}{l}\text { A: } 91,7 \\
\text { B: } 82,3\end{array}$ & k. A. & 0 & $36(18-47)$ \\
\hline Pinedo $^{\mathrm{h}}$ [325] & 2010 & 18 & $\|$ & k. A. & Retrospektiv & 100 & k. A. & 0 & 16 \\
\hline Riss [344] & 2014 & 5 & $\|$ & 20 & Retrospektiv & 0 & 0 & k. A. & k. A. \\
\hline \multicolumn{10}{|c|}{$\begin{array}{l}\text { 'Mit Internussphinkterotomie } \\
\text { 'Dauer der Fadeneinlage 9,2 } \pm 5,3 \text { Monate } \\
\text { 'Nach } 1 \text { Monat erneute Anspannung des Fadens oder Spaltung } \\
\text { dAyurvedafaden, wöchentlicher Wechsel } \\
\text { eFistelexzision ohne Sphinkterdurchtrennung } \\
\text { fPersistierende Fisteln nach Entfernung der Fadendrainage } \\
\text { 'Vergleichende Studie: A: Spülung der Drainage durch Patienten, B: konventionelle Fadendrainage } \\
\text { hZusätzlich Exzision des inneren Fistelostiums und Internusspaltung unterhalb der Linea dentata, Endpunkt: komplette Migration des Fadens nach } 4 \text { (3-12) }\end{array}$} \\
\hline
\end{tabular}

sation und fand eine direkte Korrelation. Am häufigsten waren Drüsen und Fisteln im linken unteren Quadranten aufzufinden.

Nicht in allen Fällen ist eine Einteilung von Analfisteln nach dieser Klassifikation möglich. Die Häufigkeit von unklassifizierbaren Fisteln wird mit 3-8\% angegeben $[218,348]$. Die fehlende Klassifikation erklärt sich v. a. aus der Unmöglichkeit, das innere Fistelostium zu identifizieren [218, 419]. Die Rezidivrate dieser Fisteln ist höher als bei primär klassifizierbaren Fisteln mit Nachweis einer inneren Fistelöffnung [418].

Einen speziellen Stellenwert hat die sog. Hufeisenfistel. Diese ist definiert als eine Fistel mit 2 Öffnungen in der Haut, die gemeinsam im Analkanal münden. Die häufigste Lokalisation ist dorsal mit einer inneren Öffnung bei 6.00 SSL. In einer retrospektiven Auswertung des ei- genen Krankenguts $(n=1876)$ fanden de Parades et al. [99] 82 Hufeisenfisteln (4,4\%). Davon waren 72 der Patienten männlich und $65 \%$ der Fisteln posterior lokalisiert. Die Ätiologie zeigte in $72 \%$ der Fälle eine kryptoglanduläre Ursache und in $24 \%$ der Fälle einen M. Crohn. Die Rate der voroperierten Fisteln war sehr hoch.

Eine weitere Sonderform wird von van Onkelen et al. [456] als intersphinktäre 


\begin{tabular}{|c|c|c|c|c|c|c|c|c|c|}
\hline Autor & Jahr & $\begin{array}{l}\text { Anzahl } \\
(n)\end{array}$ & Fisteltyp & $\begin{array}{l}\text { Anteil } \\
\text { M. Crohn } \\
(\%)\end{array}$ & Studiendesign & Heilung (\%) & Rezidiv (\%) & $\begin{array}{l}\text { Kontinenz- } \\
\text { störung (\%) }\end{array}$ & $\begin{array}{l}\text { Follow-up } \\
\text { (Monate) }\end{array}$ \\
\hline Parks [314] & 1976 & 30 & II, III & k. A. & Retrospektiv & k. A. & k. A. & 39 & $>12$ \\
\hline Ramanajan [335] & 1983 & 45 & III & k. A. & Retrospektiv & 98 & k. A. & 2 & 6 \\
\hline Kuypers [224] & 1984 & 10 & IV & k. A. & Retrospektiv & 100 & k. A. & 70 & $6-30$ \\
\hline Thompson [427] & 1989 & 19 & II, III & 0 & $\begin{array}{l}\text { Retrospektiv } \\
\text { VG: Tab. } 5\end{array}$ & 100 & k. A. & 68 & $16(1-47)$ \\
\hline Fasth [126] & 1990 & 7 & III, IV & k. A. & Retrospektiv & 100 & k. A. & 0 & $9-69$ \\
\hline Williams [484] & 1991 & 24 & II, III & 0 & $\begin{array}{l}\text { Retrospektiv } \\
\text { VG: } 0 \text { Tab. } 5 \\
\text { und } 7\end{array}$ & 92 & k. A. & 58 & $24(4-60)$ \\
\hline Pearl [317] & 1993 & 65 & „Komplex" & 4 & Retrospektiv & 97 & k. A. & 4,6 & 23 \\
\hline van Tets [459] & 1995 & 29 & II, IV & 0 & Retrospektiv & 87 & k. A. & $\begin{array}{l}\text { Minor: } 56, \\
\text { major: } 42\end{array}$ & k. A. \\
\hline Galis-Rozen [130] & 2008 & 60 & II, III & 0 & Retrospektiv & 60 & k. A. & 7 & 48 \\
\hline Zhang $^{\mathrm{a}}$ [497] & 2011 & $\begin{array}{l}A: 215 \\
B: 223\end{array}$ & II, III & 0 & Retrospektiv & $\begin{array}{l}78 \\
94\end{array}$ & k. A. & 0 & $>18$ \\
\hline Kelly [206] & 204 & 200 & I-IV & k. A. & $\begin{array}{l}\text { Retrospektiv, } \\
\text { multizentrisch }\end{array}$ & $\begin{array}{l}93 \\
\text { Sekundäre } \\
\text { Spaltung: } 7\end{array}$ & 6 & 0 & 6 \\
\hline Saigusa [357] & 2015 & 1 & $\begin{array}{l}\text { IV (nach } \\
\text { Prokto- } \\
\text { kolekto- } \\
\text { mie) }\end{array}$ & 100 & Fallstudie & 100 & k. A. & 0 & 12 \\
\hline
\end{tabular}

Fistel mit hoher kranialer Ausdehnung (intersphinktär oder suprasphinktär) beschrieben. Als Therapie wird eine Deckung mittels Verschiebelappen in Verbindung mit einer Abszessdrainage empfohlen (Heilung 79\%).

Fazit für die Praxis. Kryptoglanduläre Analfisteln können nach ihrer Beziehung zum Sphinkterapparat in intersphinktäre, transsphinktäre und suprasphinktäre Analfisteln eingeteilt werden. Die außerhalb des Schließmuskels gelegenen subanodermalen und extrasphinktären Fisteln sind keine typischen Analfisteln. Eine Veränderung der Leitlinienempfehlungen durch neue Studien gegenüber der Erstfassung ergibt sich nicht.

- Evidenzlevel: 4

- Empfehlungsgrad: 0

- Konsensusstärke: starker Konsens

\section{Symptomatik und Diagnostik}

\section{Basisdiagnostik}

Die typischen Symptome der Analfistel bestehen in der persistierenden Absonderung von zum Teil putridem Sekret. Bei intermittierender Abheilung der äußeren Fistelöffnung kommt es zu einem Sistieren der Sekretion, die nach Druckerhöhung, welche sich durch lokale Schmerzen bemerkbar macht, wieder auftritt. Auch bei unklaren vulvären Symptomen wurde eine Fistel als mögliche Ursache beschrieben [229].

Wichtigste diagnostische Maßnahmen sind die digitale Untersuchung und die Sondierung der Fistel. Bei einem Teil der Patienten kann der Verlauf des Fistelgangs als harter Strang getastet werden. Während unkomplizierte intersphinktäre Fisteln ihre äußere Öffnung i. d. R. direkt am Analkanal aufweisen, steigt die Anzahl komplexer (hoher) Analfisteln mit dem Abstand des äußeren Fistelostiums vom Analrand bzw. der Linea dentata [35]. Da mit dem Nachweis eines äußeren Fistelostiums meist die Operationsindikation gegeben ist, sind bei Ersteingriffen keine weiteren Untersuchungen präoperativ erforderlich. Eine Prokto- oder Rektoskopie lediglich zur Fistelsuche (inneres Fistelostium) ist nicht erforderlich oder sinnvoll.

Die weiteren Maßnahmen erfolgen intraoperativ in Narkose. Durch Sondierung kann der Verlauf des Gangs und seine Beziehung zum Schließmuskel beurteilt werden. Ist die Fistel primär nicht $\mathrm{zu}$ sondieren, gelingt es durch Anfärben des Fistelgangs mit einer Farblösung (z. B. Betaisodona-Lösung, Toluidinblau) meist, das innere Fistelostium zu lokalisieren [148, 377]. Wichtig ist die Kenntnis bogig und verschlungen verlaufender Gänge.

Folgende Faktoren können Schwierigkeiten bei der exakten Diagnostik bereiten [9]:

- Darstellung des inneren Fistelostiums nicht möglich,

- Sondierung nicht möglich,

- ungewöhnliche Anatomie,

- Fistel ohne äußeres Fistelostium, 
Tab. 7 Ergebnisse der Methode des schneidenden Fadens

\begin{tabular}{|c|c|c|c|c|c|c|c|c|c|c|}
\hline Autor & Jahr & $\begin{array}{l}\text { Anzahl } \\
(n)\end{array}$ & Fisteltyp & $\begin{array}{l}\text { Anteil } \\
\text { M. Crohn } \\
(\%)\end{array}$ & Studiendesign & $\begin{array}{l}\text { Heilungs- } \\
\text { dauer } \\
\text { (Wochen) }\end{array}$ & $\begin{array}{l}\text { Heilung } \\
\text { (\%) }\end{array}$ & $\begin{array}{l}\text { Rezidive } \\
\text { (\%) }\end{array}$ & $\begin{array}{l}\text { Kontinenz- } \\
\text { störung } \\
\text { (\%) }\end{array}$ & $\begin{array}{l}\text { Follow-up } \\
\text { (Monate) }\end{array}$ \\
\hline $\begin{array}{l}\text { Deshpande }^{a} \\
\text { [102] }\end{array}$ & 1976 & 48 & I, II, III & k. A. & Retrospektiv & $4-12$ & 96 & k. A. & k. A. & $12-96$ \\
\hline Hanley [172] & 1976 & 41 & $\begin{array}{l}\text { II (Hufei- } \\
\text { senfistel) }\end{array}$ & k. A. & Retrospektiv & k. A. & 100 & k. A. & k. A. & k. A. \\
\hline Culp [88] & 1984 & 20 & $\begin{array}{l}\text { II (distal), } \\
\text { III }\end{array}$ & k. A. & Retrospektiv & k. A. & 100 & k. A. & 15 & $>24$ \\
\hline $\begin{array}{l}\text { Vasilewsky } \\
\text { [461] }\end{array}$ & 1984 & 21 & I, II, III, IV & 6 & Retrospektiv & $<4->26$ & 93,7 & k. A. & 39 & $<25$ \\
\hline Held [174] & 1986 & 9 & ॥ & k. A. & Retrospektiv & 7 & 100 & 0 & 0 & $36(3-120)$ \\
\hline $\begin{array}{l}\text { Christensen } \\
\text { [71] }\end{array}$ & 1986 & 21 & II (distal) & k. A. & Retrospektiv & $1(<1-2)$ & 100 & 0 & $\begin{array}{l}\text { Minor: } 29, \\
\text { major: } 43\end{array}$ & $96(24-168)$ \\
\hline Misra [267] & 1988 & 59 & I, II, III & k. A. & Retrospektiv & $4(<1-7)$ & 96 & k. A. & 0 & $22(11-37)$ \\
\hline Ustynoski [443] & 1990 & 24 & II & k. A. & Retrospektiv & $7(2-17)$ & 81,4 & k. A. & k. A. & $48(8-144)$ \\
\hline Shukla [390] & 1991 & $\begin{array}{l}256(A) \\
237(B)\end{array}$ & I-IV & k. A. & Randomisiert $^{\mathrm{c}}$ & $A>B$ & 100 & $\begin{array}{l}4 \\
11\end{array}$ & $\begin{array}{l}3,0 \\
5,5\end{array}$ & 12 \\
\hline Williams [484] & 1991 & 13 & II, III & 0 & $\begin{array}{l}\text { Retrospektiv } \\
\text { VG: } \bullet \text { Tab. } 5 \\
\text { und } 7\end{array}$ & $16(8-26)$ & 100 & k. A. & $\begin{array}{l}\text { Minor: } 54 \text {, } \\
\text { major: } 7\end{array}$ & $24(4-60)$ \\
\hline Van Tets [458] & 1994 & 312 & I-IV & 0 & $\begin{array}{l}\text { Retrospektiv } \\
\text { VG: } 0 \text { Tab. } 4\end{array}$ & k. A. & k. A. & k. A. & $\begin{array}{l}\text { Grad 2: } 50 \\
\text { Grad 4: } 57\end{array}$ & k. A. \\
\hline $\begin{array}{l}\text { García Olmo } \\
\text { [132] }\end{array}$ & 1994 & 12 & II, III, IV & k. A. & Retrospektiv & $3(2-5)$ & 100 & k. A. & 25 & $6-24$ \\
\hline $\begin{array}{l}\text { McCourtney } \\
{[257]}\end{array}$ & 1996 & 27 & $\begin{array}{l}\text { I, II, III, } \\
\text { RVF, Rezi- } \\
\text { div }\end{array}$ & $15(4 / 27)$ & Retrospektiv & $20(4-76)$ & 96 & k. A. & $\begin{array}{l}19 \text { (nur } \\
\text { RVF) }\end{array}$ & $>12$ \\
\hline Walfisch [468] & 1997 & 23 & II (distal) & k. A. & Retrospektiv & 8 & 100 & k. A. & 0 & 24 \\
\hline $\begin{array}{l}\text { Hämäläinen } \\
\text { [166] }\end{array}$ & 1997 & 35 & II, III, IV & k. A. & Retrospektiv & $12(3-23)$ & 94 & k. A. & 63 & $70(28-184)$ \\
\hline Dziki [111] & 1998 & 33 & $\begin{array}{l}\text { II (distal), } \\
\text { III, IV }\end{array}$ & k. A. & Retrospektiv & $12(0-22)$ & 100 & k. A. & $\begin{array}{l}\text { Minor: } 34, \\
\text { major: } 38\end{array}$ & $16(4-22)$ \\
\hline $\begin{array}{l}\text { Garcia-Aguilar } \\
\text { [133] }\end{array}$ & 1998 & 12 & II, III & k. A. & $\begin{array}{l}\text { Retrospektiv } \\
\text { VG: • Tab. } 5\end{array}$ & $16(8-36)$ & 92 & k. A. & $\begin{array}{l}\text { Minor: } 67 \\
\text { major: } 25\end{array}$ & 27 \\
\hline Isbister [193] & 2001 & 47 & ॥ & k. A. & Retrospektiv & k. A. & 98 & k. A. & $\begin{array}{l}\text { Grad 1: } 36, \\
\text { Grad 2: } 8,4, \\
\text { Grad 3: } 2,3\end{array}$ & 13 \\
\hline Ho [182] & 2001 & 46 & $\begin{array}{l}\text { I, II (proxi- } \\
\text { mal) }\end{array}$ & 0 & $\begin{array}{l}\text { Randomisiert }^{\mathrm{d}} \\
\text { VG: • Tab. } 4\end{array}$ & 8 & 100 & k. A. & 11 & $\begin{array}{l}2,2 \\
(0,6-9,2)\end{array}$ \\
\hline Mylonakis [279] & 2001 & 28 & II, III & 0 & $\begin{array}{l}\text { Retrospektiv } \\
\text { VG: - Tab. } 4\end{array}$ & k. A. & 93 & k. A. & 11 & 3 \\
\hline Theerapol [424] & 2002 & 41 & I, II, III & k. A. & $\begin{array}{l}\text { Retrospektiv } \\
\text { VG: } 0 \text { Tab. } 4\end{array}$ & $9(4-26)$ & 78 & 5,4 & 0 & $4(0,5-17)$ \\
\hline Joy [203] & 2002 & 17 & $\begin{array}{l}\text { II (proxi- } \\
\text { mal) }\end{array}$ & k. A. & $\begin{array}{l}\text { Retrospektiv } \\
\text { VG: } \bullet \text { Tab. } 5 \\
\text { und } 10\end{array}$ & 20 & 94 & k. A. & $\begin{array}{l}\text { Minor: } 50, \\
\text { major: } 20\end{array}$ & $19(9-54)$ \\
\hline Durgun $^{e}[109]$ & 2002 & 10 & IV & k. A. & Retrospektiv & k. A. & 100 & k. A. & 20 & $3-108$ \\
\hline Zbar [496] & 2003 & $\begin{array}{l}18(\mathrm{~A}) \\
16(\mathrm{~B})\end{array}$ & II & 0 & Randomisiert ${ }^{\mathrm{f}}$ & $\begin{array}{l}14(6-38) \\
12(4-28)\end{array}$ & $\begin{array}{l}100 \\
100\end{array}$ & $\begin{array}{l}11 \\
6,6\end{array}$ & $\begin{array}{l}6 \\
13\end{array}$ & $\begin{array}{l}13(6-30) \\
12(5-28)\end{array}$ \\
\hline Mentes [265] & 2004 & 20 & ॥ & 0 & Retrospektiv & $<3(1-4)$ & $\begin{array}{l}1 \text { Monat: } 45 \\
3 \text { Monat: } \\
100\end{array}$ & k. A. & 20 & $12(6-24)$ \\
\hline Pagano [304] & 2004 & 54 & II, III, IV & 0 & Retrospektiv & k. A. & 90,7 & k. A. & 11 & k. A. \\
\hline Hamel [167] & 2004 & 12 & ॥ & k. A. & Retrospektiv & k. A. & 100 & k. A. & 0 & k. A. \\
\hline
\end{tabular}


Tab. 7 Ergebnisse der Methode des schneidenden Fadens (Fortsetzung)

\begin{tabular}{|c|c|c|c|c|c|c|c|c|c|c|}
\hline Autor & Jahr & $\begin{array}{l}\text { Anzahl } \\
(n)\end{array}$ & Fisteltyp & $\begin{array}{l}\text { Anteil } \\
\text { M. Crohn } \\
\text { (\%) }\end{array}$ & Studiendesign & $\begin{array}{l}\text { Heilungs- } \\
\text { dauer } \\
\text { (Wochen) }\end{array}$ & $\begin{array}{l}\text { Heilung } \\
(\%)\end{array}$ & $\begin{array}{l}\text { Rezidive } \\
(\%)\end{array}$ & $\begin{array}{l}\text { Kontinenz- } \\
\text { störung } \\
\text { (\%) }\end{array}$ & $\begin{array}{l}\text { Follow-up } \\
\text { (Monate) }\end{array}$ \\
\hline Pescatori [321] & 2004 & 17 & II & $6(5 / 85)$ & Retrospektiv & k. A. & 94,7 & k. A. & $\begin{array}{l}\text { Minor: } 6, \\
\text { major: } 12\end{array}$ & $22(5-89)$ \\
\hline $\begin{array}{l}\text { Hammond } \\
{[169]}\end{array}$ & 2006 & 29 & I, II & 0 & Retrospektiv & 24 & $52^{9}$ & 0 & 34 & 42 \\
\hline Vatansev [463] & 2007 & 32 & II, III & 0 & Retrospektiv & $8(6-10)$ & 100 & k. A. & 16 & 26 \\
\hline Gürer [161] & 2007 & 17 & I, II, III & 0 & Retrospektiv & 2,5 & 100 & k. A. & 0 & 8,2 \\
\hline $\begin{array}{l}\text { Chuang-Wei } \\
\text { [76] }\end{array}$ & 2008 & 112 & II, III, IV & k. A. & Retrospektiv & 9,3 & 100 & 0,9 & $\begin{array}{l}24,1 \\
\text { Grad 1: } \\
\text { 18,6, } \\
\text { Grad 2: } \\
\text { 5,4, Grad 3: } \\
0\end{array}$ & 39 \\
\hline Browder $^{h}$ [47] & 2009 & 23 & II, III & 0 & Retrospektiv & $8 \pm 5$ & 81 & k. A. & 0 & 15 \\
\hline Roig [349] & 2009 & 30 & I-IV & 0 & $\begin{array}{l}\text { Retrospektiv } \\
\text { VG: - Tab. } 10 \\
\text { und } 12\end{array}$ & k. A. & 83,3 & k. A. & k. A. & $19 \pm 25$ \\
\hline Altomare [20] & 2010 & 24 & II & 0 & $\begin{array}{l}\text { Randomisiert } \\
\text { VG: - Tab. } 14\end{array}$ & 12 & 88 & k. A. & $\begin{array}{l}\text { Wexner- } \\
\text { Score } \\
2=>5\end{array}$ & 12 \\
\hline Subhas $[414]$ & 2010 & 2 & II & 25 & Retrospektiv & 60 & $\begin{array}{l}75 \text { (Rest } \\
\text { gespalten) }\end{array}$ & 0 & $\begin{array}{l}\text { Grad 2: 8, } \\
\text { Grad 2/3: } 0\end{array}$ & $12-81$ \\
\hline Memon $^{k}[263]$ & 2011 & 79 & II, III & k. A. & Retrospektiv & $11 \pm 6$ & 95 & k. A. & 0 & $>12$ \\
\hline $\operatorname{Han}^{\prime}[171]$ & 2011 & 40 & $\|$ & 0 & Retrospektiv & k. A. & 100 & k. A. & $75(30 / 40)$ & 6 \\
\hline Tokunaga [431] & 2013 & $\begin{array}{l}239 \\
\text { II: } 198 \\
\text { III: } 41\end{array}$ & II, III & k. A. & Retrospektiv & $\begin{array}{l}\text { II: } 6 \\
\text { III: } 20\end{array}$ & $\begin{array}{l}\text { II: } 100 \\
\text { III: } 95\end{array}$ & k. A. & $\begin{array}{l}\text { II: } 0,5 \\
(1 / 198) \\
\text { III: } 7(3 / 41)\end{array}$ & $18-36$ \\
\hline Ege [114] & 2014 & 128 & II & 0 & Retrospektiv & $1,5-4,3$ & 100 & 1,5 & $\begin{array}{l}\text { Keine sig- } \\
\text { nifikante } \\
\text { Änderung } \\
\text { des Scores }\end{array}$ & $>12$ \\
\hline Patton [316] & 2015 & 59 & $\begin{array}{l}\text { I: } 17 \% \\
\text { II: } 66 \% \\
\text { III: } 3,4 \% \\
\text { IV: } 3,4 \%\end{array}$ & 0 & Retrospektiv & k. A. & $\begin{array}{l}93 \text { (primär) } \\
98 \text { (sekun- } \\
\text { där) }\end{array}$ & 6,8 & $\begin{array}{l}63 \text { gleich } \\
\text { oder besser, } \\
\text { Verschlech- } \\
\text { terung v. a. } \\
\text { bei Frauen }\end{array}$ & $\begin{array}{l}9,4 \\
(1,7-5,6)\end{array}$ \\
\hline Dutta $^{m}$ [110] & 2015 & 26 & $\begin{array}{l}\text { I }(74 \%), \text { II } \\
(26 \%)\end{array}$ & k. A. & $\begin{array}{l}\text { Randomisiert } \\
\text { VG: • Tab. } 4\end{array}$ & $8 \pm 4$ & 100 & k. A. & 4 & k. A. \\
\hline Rosen [354] & 2016 & 121 & II & 0 & Retrospektiv & $3(1-18)$ & 93 & k. A. & $\begin{array}{l}7,5 \\
\text { (Inkontinenz- } \\
\text { rate der } \\
\text { gesamten } \\
\text { Gruppe } \\
\text { sank von } 19 \\
\text { auf } 11 \% ! ?)\end{array}$ & $5(1-24)$ \\
\hline \multicolumn{11}{|c|}{ 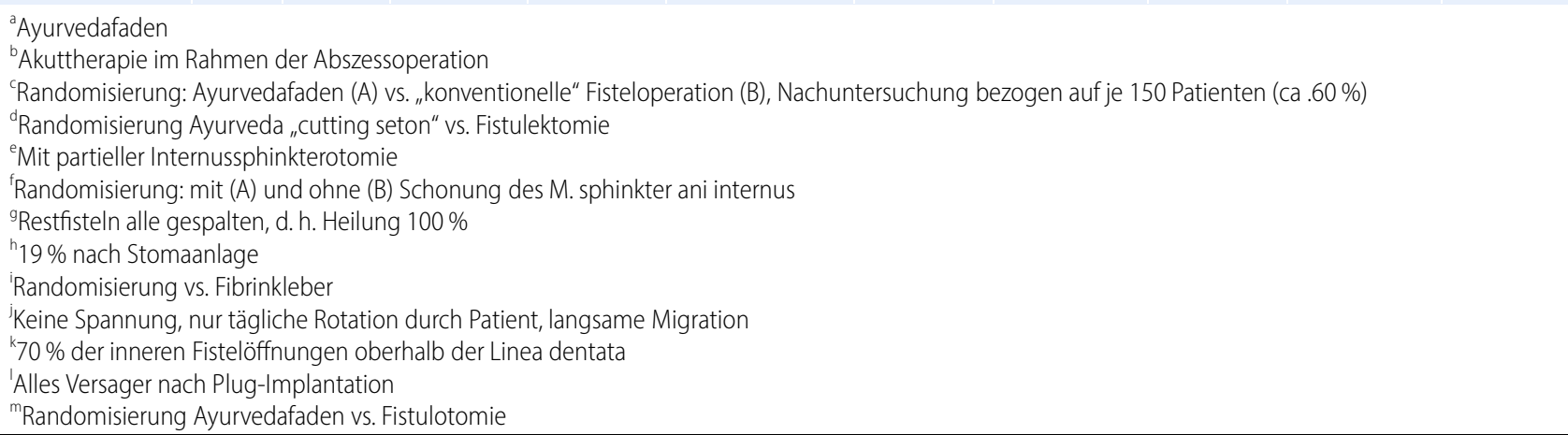 } \\
\hline
\end{tabular}


Tab. 8 Ergebnisse der Fistelexzision mit primärer Naht des inneren Ostiums ohne Flap

\begin{tabular}{|c|c|c|c|c|c|c|c|c|c|}
\hline Autor & Jahr & $\begin{array}{l}\text { Anzahl } \\
\text { (n) }\end{array}$ & Fisteltyp & $\begin{array}{l}\text { Anteil } \\
\text { M. Crohn } \\
(\%)\end{array}$ & Studiendesign & Heilung (\%) & Rezidive (\%) & $\begin{array}{l}\text { Kontinenz- } \\
\text { störung (\%) }\end{array}$ & $\begin{array}{l}\text { Follow-up } \\
\text { (Monate) }\end{array}$ \\
\hline Ackermann $^{\mathrm{a}}[10]$ & 1994 & 12 & Ilb & k. A. & Retrospektiv & 67 & k. A. & 0 & k. A. \\
\hline Thomson [428] & 2003 & 44 & ॥ & $14(6 / 44)$ & Retrospektiv & 59 & 11 & 10 & 91 (19-146) \\
\hline $\begin{array}{l}\text { Athanasiadis } \\
\text { [25] }\end{array}$ & 2004 & 90 & II, Rezidiv & 0 & Prospektiv & 87 & 10 & 6 & $30(6-60)$ \\
\hline Köhler [219] & 2004 & 11 & $\begin{array}{l}\text { II (Hufeisen- } \\
\text { fistel) }\end{array}$ & 0 & $\begin{array}{l}\text { Prospektiv } \\
\text { VG: } \\
\text { - Tab. } 9,10 \\
\text { und } 11\end{array}$ & 100 & 0 & k. A. & $58(12-168)$ \\
\hline Tobisch [430] & 2012 & 262 & $|-|||$ & 0 & Retrospektiv & 74 & k. A. & $\begin{array}{l}44 \\
55 \text { (nicht } \\
\text { voroperierte } \\
\text { Fisteln) }\end{array}$ & $70(14-141)$ \\
\hline
\end{tabular}

- verzweigte Fisteln,

- vorgeschädigter Analkanal.

Bei entsprechenden Verdachtsmomenten in der Anamnese sollte perioperativ der Ausschluss einer chronisch-entzündlichen Darmerkrankung erfolgen.

Die Schließmuskelfunktion sollte vor einer operativen Intervention anhand der Anamnese und ggf. eines Inkontinenzscores eingeschätzt werden. Die Manometrie liefert keine zusätzliche Information und ist als Routineuntersuchung entbehrlich. Der diagnostische und therapeutische Algorithmus wird in - Abb. 3 dargestellt.

Fazit für die Praxis. Der Nachweis einer äußeren Fistelöffnung sollte die Indikation zur operativen Intervention darstellen. Die endgültige Klassifikation sollte intraoperativ anhand der klinischen Untersuchung, Sondierung und ggf. Anfärbung erfolgen. Eine präoperative klinische Beurteilung der Sphinkterfunktion ist sinnvoll. Eine Veränderung der Leitlinienempfehlungen durch neue Studien gegenüber der Erstfassung ergibt sich nicht.

- Evidenzlevel: 4

- Empfehlungsgrad: 0

- Konsensusstärke: starker Konsens

\section{Weiterführende Diagnostik}

Eine bildgebende Diagnostik ist nur in Ausnahmefällen erforderlich. Eine Indikation kann sich insbesondere bei trans- und suprasphinktären Rezidivfisteln ergeben. In einigen Fällen dient die Bildgebung auch dem Ausschluss einer chronisch-entzündlichen Darmerkrankung.

Möglichkeiten der Bildgebung bestehen in der radiologischen Fisteldarstellung (Fistulographie), der Endosonographie und der Schichtuntersuchung der Perianalregion mittels Magnetresonanztomographie (MRT) oder Computertomographie (CT). Die Aussagekraft der Bildgebung ist dabei in hohem Maße von der technischen Qualität der Untersuchung abhängig. So finden ältere Studien im Vergleich klinische Untersuchung versus Endosonographie [68] eine höhere Aussagekraft der Klinik, während neuere Studien [436] deutliche Vorteile der Endosonographie, insbesondere beim Nachweis von Hufeisenfisteln und für die exakte Lokalisation des inneren Fistelostiums sehen.

Die radiologische Darstellung der Fistel [477] ist auch wegen der Strahlenbelastung heute im Rahmen einer standardisierten Diagnostik als obsolet anzusehen. Schon eine Arbeit aus dem Jahr 1985 [223] konnte eine exakte Voraussage des Fistelverlaufs bei nur $16 \%$ der Untersuchten darstellen. Lediglich eine Publikation aus dem Jahr 2010 [326] weist die Fistulographie als einfache und kostengünstige Methode der ersten Wahl aus. Die Sensitivität der Fistulographie wird mit $91 \%$, die Spezifität mit $100 \%$ bestimmt. Die Übereinstimmung mit der Narkoseuntersuchung war in allen Fällen gegeben. Eine Abgrenzung zur Endoso- nographie und MRT erfolgte in dieser Arbeit jedoch nicht.

Als technisch einfaches und kostengünstiges Verfahren wurde die Endosonographie seit den 1980er Jahren in mehreren Publikationen vorgestellt [225]. Durch die Kontrastierung der Fistel mit Wasserstoffperoxid kann die Aussage verbessert werden [51, 209, 250, $275,282]$, wobei die Instillation von den Patienten z.T. als unangenehm empfunden wird. Die Korrelation zwischen endoanalem Ultraschall und der intraoperativen klinischen Untersuchung liegt bei über $90 \%$ [56, 231, 250, 337]. Eine Beeinflussung des operativen Vorgehens in erster Linie im Sinne eines mehr konservativen Vorgehens wird von Lindsey et al. [237] bei $38 \%$ der Patienten beschrieben, während Weisman et al. [476] keine Beeinflussung des perioperativen Verlaufs durch die Endosonographie nachweisen konnten. Subasighe et al. [413] sehen die Bedeutung der Endosonographie v. a. im Nachweis von nicht drainiertem Sekretverhalten vor einer Fisteloperation. Kritisch muss hier angemerkt werden, dass die Studien oft ein gemischtes Krankengut unter Einbeziehung von Rezidivfisteln und Fisteln bei chronisch-entzündlichen Darmerkrankungen untersuchen, so dass die Aussagen im Rahmen der Fistelerstbehandlung nur eingeschränkt zu bewerten sind.

Eine Weiterentwicklung stellt die 3DEndosonographie dar. Santoro et al. [362] konnten das innere Fistelostium mit der 


\begin{tabular}{|c|c|c|c|c|c|c|c|c|c|}
\hline Autor & Jahr & $\begin{array}{l}\text { Anzahl } \\
\text { (n) }\end{array}$ & Fisteltyp & $\begin{array}{l}\text { Anteil } \\
\text { M. Crohn } \\
(\%)\end{array}$ & Studiendesign & Heilung (\%) & $\begin{array}{l}\text { Rezidive } \\
(\%)\end{array}$ & $\begin{array}{l}\text { Kontinenz- } \\
\text { störung (\%) }\end{array}$ & $\begin{array}{l}\text { Follow-up } \\
\text { (Monate) }\end{array}$ \\
\hline Oh [287] & 1983 & 15 & $\begin{array}{l}\text { II, III } \\
\text { (Rezi- } \\
\text { div) }\end{array}$ & k. A. & Retrospektiv & 86,7 & k. A. & k. A. & k. A. \\
\hline Aguilar [15] & 1985 & 151 & $\begin{array}{l}\text { II, III, } \\
\text { Rezidiv }\end{array}$ & k. A. & Retrospektiv & k. A. & 1,5 & 10 & $8-84$ \\
\hline Wedell $^{\mathrm{a}}[475]$ & 1987 & 30 & II, RVF & k. A. & Retrospektiv & 97 & 0 & $0^{\mathrm{a}}$ & $18-48$ \\
\hline Girona [5] & 1987 & $\begin{array}{l}70 \\
26\end{array}$ & $\begin{array}{l}\text { II } \\
\text { III }\end{array}$ & 0 & Retrospektiv & $\begin{array}{l}75 \\
80\end{array}$ & $\begin{array}{l}3 \\
8\end{array}$ & $\begin{array}{l}\text { Minor: } 8 \\
\text { major: } 19\end{array}$ & 36 \\
\hline Shemesh [388] & 1988 & 8 & $\begin{array}{l}\text { II, III } \\
\text { (anteri- } \\
\text { or) }\end{array}$ & 0 & Retrospektiv & 87,5 & k. A. & k. A. & k. A. \\
\hline Lechner $^{b}$ [227] & 1991 & 28 & $\begin{array}{l}\text { II (dis- } \\
\text { tal), } \\
\text { III }\end{array}$ & $7(2 / 28)$ & Retrospektiv & 100 & 0 & 0 & 8,1 \\
\hline Kodner [215] & 1993 & $\begin{array}{l}24 \\
31\end{array}$ & $\begin{array}{l}\text { II, RVF } \\
\|\end{array}$ & $\begin{array}{l}100 \\
0\end{array}$ & Retrospektiv & $\begin{array}{l}71 \\
79\end{array}$ & k. A. & k. A. & k. A. \\
\hline Kodner [215] & 1993 & 31 & II & 0 & Retrospektiv & 79 & k. A. & k. A. & k. A. \\
\hline Athanasiadis [26] & 1994 & 189 & $\begin{array}{l}\text { II, Rezi- } \\
\text { div }\end{array}$ & 0 & Retrospektiv & 91 & k. A. & 21 & $12-90$ \\
\hline Athanasiadis [26] & 1994 & 35 & $\begin{array}{l}\text { III, } \\
\text { Rezidiv }\end{array}$ & 0 & Retrospektiv & 80 & k. A. & 43 & $12-90$ \\
\hline Athanasiadis [27] & 1995 & 18 & $\begin{array}{l}\text { II, Rezi- } \\
\text { div }\end{array}$ & 14 & $\begin{array}{l}\text { Retrospektiv } \\
\text { VG: } 0 \text { Tab. } 10\end{array}$ & 67 & 9,5 & k. A. & 11 \\
\hline Golub c [147] & 1997 & 164 & II-IV & 0 & Retrospektiv & 100 & $3(2 / 61)$ & 15 & $71(19-135)$ \\
\hline Schouten [369] & 1999 & 44 & II, III & 0 & Retrospektiv & $\begin{array}{l}75 \\
\text { Rez. Fisteln: } 50\end{array}$ & k. A. & 35 & 12 \\
\hline D’Agostino [89] & 2000 & 21 & II, RVF & $19(4 / 21)$ & Retrospektiv & 76 & k. A. & 0 & 20 \\
\hline Zimmermann [499] & 2003 & 105 & II & 0 & Prospektiv & 69 & k. A. & k. A. & 14 \\
\hline Zmora ${ }^{e}[503]$ & 2003 & 13 & $\begin{array}{l}\text { II, RVF, } \\
\text { Div. }\end{array}$ & $15(2 / 13)$ & $\begin{array}{l}\text { Retrospektiv } \\
\text { VG: - Tab. } 14\end{array}$ & 54 & k. A. & k. A. & $12(1-36)$ \\
\hline Dixon [105] & 2004 & 29 & II, RVF? & k. A. & Retrospektiv & 69 & k. A. & k. A. & 3 \\
\hline Köhler [219] & 2004 & 15 & $\begin{array}{l}\text { II (Huf- } \\
\text { eisen- } \\
\text { fistel) }\end{array}$ & 0 & $\begin{array}{l}\text { Prospektiv } \\
\text { VG: } \\
\text { - Tab. } 8,10 \\
\text { und } 11\end{array}$ & 60 & 25 & k. A. & $58(12-168)$ \\
\hline $\begin{array}{l}\text { Van der Hagen }{ }^{f} \\
\text { [444] }\end{array}$ & 2005 & 30 & II, III, IV & 23 & Retrospektiv & 97 & 22 & 23 & $22(8-52)$ \\
\hline Ellis [120] & 2006 & 66 & II, III & 0 & $\begin{array}{l}\text { Randomisiert }^{d} \\
\text { VG: } 0 \text { Tab. } 11 \\
\text { und } 14\end{array}$ & $\begin{array}{l}83(\mathrm{~A}) \\
41(\mathrm{~B})\end{array}$ & k. A. & k. A. & 22 \\
\hline Mitalas [271] & 2007 & $\begin{array}{l}87 \\
26\end{array}$ & $\begin{array}{l}\text { II } \\
\text { II (Rezi- } \\
\text { div) }\end{array}$ & 0 & Retrospektiv & $\begin{array}{l}67 \\
69\end{array}$ & k. A. & 0 & $15(2-50)$ \\
\hline Alexander ${ }^{9}[19]$ & 2008 & 8 & $\begin{array}{l}\text { II (dis- } \\
\text { tal) }\end{array}$ & 0 & Prospektiv & 12,5 & k. A. & k. A. & k. A. \\
\hline Dubsky [107] & 2008 & 34 & II, III & 0 & Retrospektiv & 64,7 & k. A. & 23,5 & $63(9-118)$ \\
\hline Van Koperen [452] & 2008 & 70 & II, III & 0 & $\begin{array}{l}\text { Retrospektiv } \\
\text { VG: - Tab. } 4\end{array}$ & k. A. & 21 & 40 & $76(7-134)$ \\
\hline Abbas [2] & 2008 & 36 & $\begin{array}{l}\text { II, III, } \\
\text { RVF } \\
\text { (81\% } \\
\text { Rezidiv) }\end{array}$ & k. A. & Retrospektiv & 86 & k. A. & k. A. & 27 \\
\hline
\end{tabular}




\section{Leitlinien}

Tab. 9 Ergebnisse des Mukosa/Submukosa-Verschiebelappens (Mukosa-Advancement-Flap) (Fortsetzung)

\begin{tabular}{|c|c|c|c|c|c|c|c|c|c|}
\hline Autor & Jahr & $\begin{array}{l}\text { Anzahl } \\
(n)\end{array}$ & Fisteltyp & $\begin{array}{l}\text { Anteil } \\
\text { M. Crohn } \\
(\%)\end{array}$ & Studiendesign & Heilung (\%) & $\begin{array}{l}\text { Rezidive } \\
\text { (\%) }\end{array}$ & $\begin{array}{l}\text { Kontinenz- } \\
\text { störung (\%) }\end{array}$ & $\begin{array}{l}\text { Follow-up } \\
\text { (Monate) }\end{array}$ \\
\hline Wang [472] & 2009 & 26 & II & 0 & $\begin{array}{l}\text { Retrospektiv } \\
\text { VG: • Tab. } 17\end{array}$ & 63 & k. A. & k. A. & 27 \\
\hline Chung [77] & 2009 & 96 & II, III & 0 & $\begin{array}{l}\text { Prospektiv } \\
\text { VG: } \\
\text { - Tab. } 5,14 \\
\text { und } 17\end{array}$ & 60 & k. A. & k. A. & 3 \\
\hline $\begin{array}{l}\text { Van der Hagen } \\
\text { [445] }\end{array}$ & 2009 & 41 & II & 0 & $\begin{array}{l}\text { Prospektiv } \\
\text { VG: } 0 \text { Tab. } 14\end{array}$ & 90 & k. A. & 0 & 26 \\
\hline Mitalas [270] & 2009 & 80 & $\begin{array}{l}\text { II (dis- } \\
\text { tal) }\end{array}$ & 0 & Retrospektiv & 67,5 & 0 & k. A. & $92(52-161)$ \\
\hline Ellis [121] & 2007 & 29 & II & 0 & $\begin{array}{l}\text { Retrospektiv } \\
\text { VG: D Tab. } 11\end{array}$ & 69 & k. A. & k. A. & $10(6-22)$ \\
\hline Zbar [492] & 2010 & 11 & II, III & 0 & Prospektiv & 82 & k. A. & 18 & $20(8-42)$ \\
\hline Jordan [202] & 2010 & 26 & II-IV & 0 & $\begin{array}{l}\text { Retrospektiv } \\
\text { VG: } 0 \text { Tab. } 4 \\
\text { und } 12\end{array}$ & 84,6 & k. A. & k. A. & $19 \pm 25$ \\
\hline Muhlmann [276] & 2010 & $\begin{array}{l}48 \\
\text { Gesamt: } \\
55 \text { Patien- } \\
\text { ten } \\
70 \text { Opera- } \\
\text { tionen }\end{array}$ & $\begin{array}{l}\text { II, III, } \\
\text { RVF }\end{array}$ & 57 & $\begin{array}{l}\text { Retrospektiv } \\
\text { VG: - Tab. } 17\end{array}$ & $33(16 / 48)$ & k. A. & k. A. & $5(0,3-36)$ \\
\hline Mitalas $^{\mathrm{h}}[268]$ & 2011 & $\begin{array}{l}162 \\
\text { Anterior: } \\
\text { Hufeisen: } \\
\text { 15, direkt: } \\
23 \\
\text { Posterior: } \\
\text { Hufeisen: } \\
27 \text {, in- } \\
\text { tersphink- } \\
\text { tär: 49, } \\
\text { trans- } \\
\text { sphinktär: } \\
43\end{array}$ & II & k. A. & Retrospektiv & $\begin{array}{l}60 \\
52 \\
81 \\
73 \\
37\end{array}$ & k. A. & k. A. & $>12$ \\
\hline Khafagy [208] & 2010 & 20 & II & 0 & $\begin{array}{l}\text { Randomisiert } \\
\text { VG: }{ }^{i} \\
\text { Tab. } 10\end{array}$ & 70 & $\begin{array}{l}40 \text { (Ge- } \\
\text { samthei- } \\
\text { lung: } 30 \%)\end{array}$ & 0 & k. A. \\
\hline $\begin{array}{l}\text { Van der Hagen } \\
\text { [447] }\end{array}$ & 2011 & 15 & II, III & 0 & $\begin{array}{l}\text { Randomisiert }^{\mathrm{j}} \\
\text { VG: - Tab. } 14\end{array}$ & 80 & k. A. & 0 & 52 \\
\hline Jarrark $^{k}$ [198] & 2011 & 98 & $\begin{array}{l}\text { II, III, } \\
\text { RVF }\end{array}$ & 33 (CED 41) & Retrospektiv & $\begin{array}{l}72(54 / 98) \\
\text { Kryptoglandulär: } \\
98, \text { Crohn } 87\end{array}$ & k. A. & $\begin{array}{l}\text { Kontinenz } \\
\text { besser }\end{array}$ & $84 \pm 36$ \\
\hline Van Koperen [449] & 2011 & 30 & II, III & 0 & $\begin{array}{l}\text { Randomisiert, } \\
\text { multizentrisch } \\
\text { VG: - Tab. } 17\end{array}$ & 48 & k. A. & $\begin{array}{l}\text { Vaizey-Score } \\
\text { postop. } \\
\text { leicht erhöht } \\
\text { Wexner-Score } \\
\text { gleich }\end{array}$ & 11 \\
\hline Stremitzer' [412] & 2012 & 2 & II, III & 0 & $\begin{array}{l}\text { Fallstudie } \\
\text { VG: - Tab. } 10\end{array}$ & 50 & k. A. & k. A. & $85(26-136)$ \\
\hline Hall [165] & 2014 & 15 & I, II, RVF & 12 & $\begin{array}{l}\text { Prospektiv, } \\
\text { multizentrisch } \\
\text { (16 Operateure) } \\
\text { VC: Tab. 4, } 14 \\
\text { und } 17\end{array}$ & 60 & k. A. & $\begin{array}{l}\text { CCFI postop. } \\
\text { niedriger }\end{array}$ & 3 \\
\hline Van Onkelen ${ }^{\mathrm{m}}$ [457] & 2014 & 252 & ॥ & 0 & Retrospektiv & 59 & k. A. & k. A. & $21(6-136)$ \\
\hline
\end{tabular}




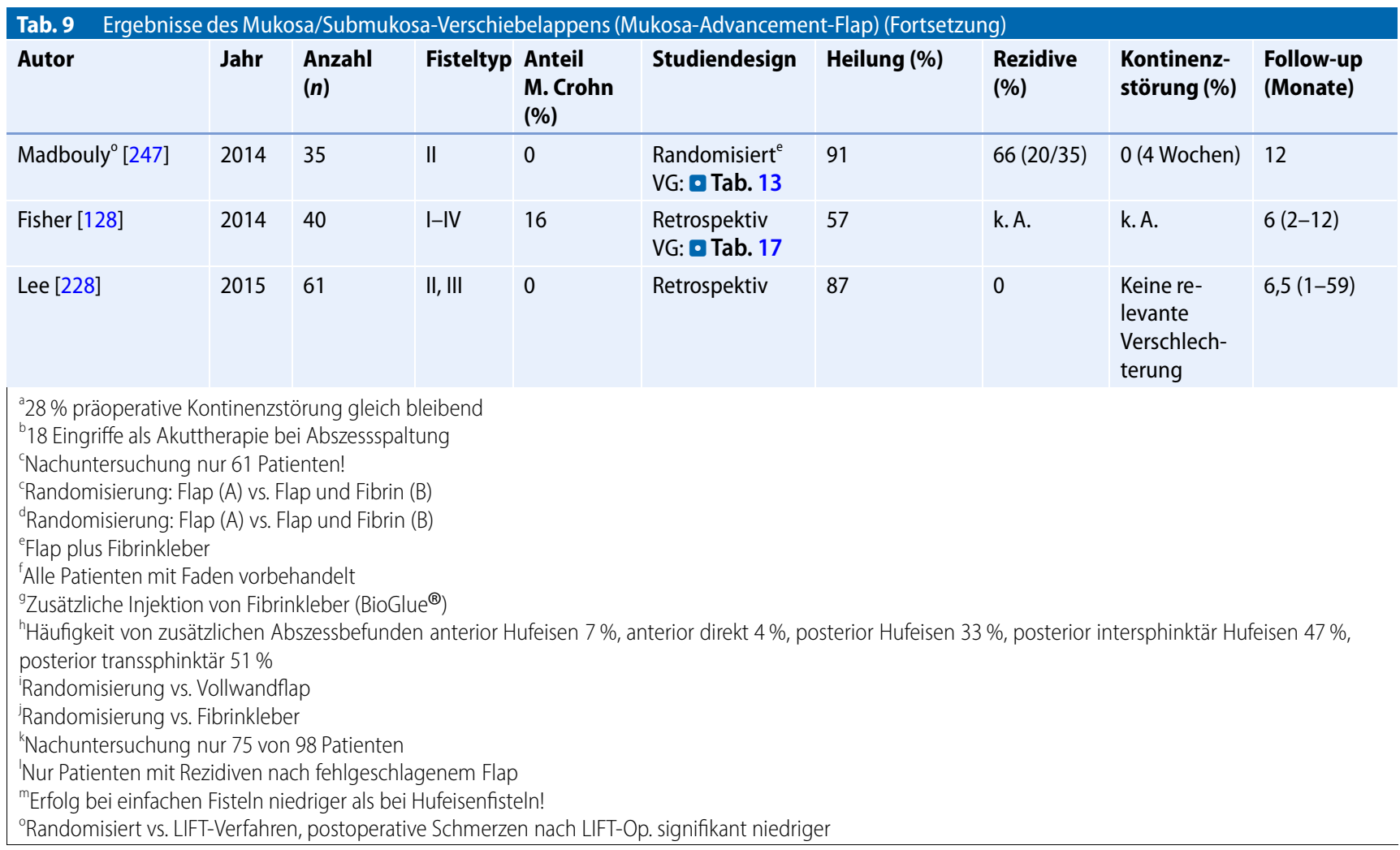

$3 \mathrm{D}$-Endosonographie in $85 \%$ gegenüber $67 \%$ bei der 2D-Endosonographie nachweisen. Ratto et al. [337] sahen ebenfalls eine höhere Genauigkeit der 3D-Endosonographie in Bezug auf die Darstellung von Primär- und Sekundärgängen und den Nachweis des inneren Fistelostiums. Zu den gleichen Ergebnissen kamen West et al. [478], ohne dass sie eine vermehrte Belastung der Patienten durch die Untersuchung beobachteten. In einer Untersuchung bei anterioren Fisteln sahen Murad-Regadas et al. [277] den Vorteil dieser Technik v. a. in der Quantifizierung des von der Fistel umschlossenen Muskels im Hinblick auf eine Spaltung.

Auch aktuelle Studien sehen Vorteile für die 3D-Endosonographie und die peroxidverstärkte Endosonographie für das Outcome v. a. bei komplexen Fisteln [39, 104, 280, 327].

Eine weitere Studie beschreibt sogar eine mögliche Differenzierung von Crohn- und kryptoglandulären Fisteln [44].

Einzelne Autoren sehen eine Alternative zur endoanalen Ultraschalluntersuchung in der transperinealen Ultraschalluntersuchung, die jedoch mit einer er- heblichen Lernkurve verbunden ist [495]. Kleinübing et al. [211] sehen diese gegenüber der klinischen Untersuchung als überlegen an und finden keinen Vorteil für eine gleichzeitige Wasserstoffperoxidinstillation.

Ein Review der vorliegenden Literatur [155] kommt zu der Schlussfolgerung, dass die 3D-Endosonographie der MRT im Nachweis von Seitengängen und Sekretverhalten sowie der Lokalisation des inneren Fistelostiums überlegen ist und in einigen Fällen das operative Prozedere beeinflussen kann.

Die MRT kann entweder als externe Untersuchung mit oder ohne Kontrastmittel oder mittels einer endorektalen Spule durchgeführt werden. Letztere steht jedoch nur in Zentren zur Verfügung und scheint keine bessere $\mathrm{Be}$ urteilung zu erlauben. Bei komplexen Fisteln und insbesondere in Verbindung mit vermuteter Höhlenbildung ist die Indikation $\mathrm{zu}$ überlegen. In den letzten Jahren konnte die Aussagekraft der MRT durch verschiedene technische Neuerungen verbessert werden [188, 365]. Chapple et al. [65] beschreiben für die kontrastmittelverstärkte MRT-
Untersuchung Vorteile im klinischen Outcome gegenüber der reinen chirurgischen Exploration. Dagegen beschreiben Scholefield et al. [368] die schwierige Interpretation der MRT-Befunde und halten die MRT nur bei komplexen Fisteln für sinnvoll. Im direkten Vergleich zwischen den endoanalen MRT-Untersuchungen und der Endosonographie beschreiben Gustafson et al. [164] gleiche Ergebnisse nach beiden Verfahren und sehen somit Vorteile für die technisch einfachere und kostengünstigere Endosonographie.

Ein Review aus einer radiologischen Klinik [356] unter Berücksichtigung evidenzbasierter Grundlagen kommt zu der Schlussfolgerung, dass das MRT auf dem Boden randomisierter Studien der klinischen Untersuchung und der Endosonographie überlegen ist (Evidenzlevel Ib) und fordert eine MRT-Untersuchung bei allen Patienten mit klinischem Fistelverdacht. Dies stellt aus Sicht der Autoren jedoch eine völlige Überdiagnostik der Mehrheit von Fisteln dar.

Ein Vergleich verschiedener bildgebender Verfahren von Sofic et al. [400] ergab eine richtige Befunderhebung 
Tab. 10 Ergebnisse des Rektumwand-Verschiebelappens (Rectal-Advancement-Flap)

\begin{tabular}{|c|c|c|c|c|c|c|c|c|c|}
\hline Autor & Jahr & $\begin{array}{l}\text { Anzahl } \\
(n)\end{array}$ & $\begin{array}{l}\text { Fistel- } \\
\text { typ }\end{array}$ & $\begin{array}{l}\text { Anteil } \\
\text { M. Crohn } \\
(\%)\end{array}$ & Studiendesign & Heilung (\%) & $\begin{array}{l}\text { Rezidive } \\
(\%)\end{array}$ & $\begin{array}{l}\text { Kontinenz- } \\
\text { störung (\%) }\end{array}$ & $\begin{array}{l}\text { Follow-up } \\
\text { (Monate) }\end{array}$ \\
\hline Reznick [342] & 1988 & 7 & II-IV & 0 & Retrospektiv & 85 & k. A. & 0 & $32 \pm 36$ \\
\hline Athanasiadis [27] & 1995 & $\begin{array}{l}23 \\
4\end{array}$ & $\begin{array}{l}\text { II (Rez.) } \\
\text { III } \\
\text { (Rez.) }\end{array}$ & 0 & $\begin{array}{l}\text { Prospektiv } \\
\text { VG: Tab. } 9\end{array}$ & $\begin{array}{l}78 \\
75\end{array}$ & $\begin{array}{l}2,9 \\
2,5\end{array}$ & k. A. & 11 \\
\hline Ozuner [303] & 1996 & 19 & II, III & 0 & Retrospektiv & 68,4 & k. A. & k. A. & $31(1-79)$ \\
\hline Miller [266] & 1998 & 21 & II, III & 0 & Retrospektiv & 81 & k. A. & 0 & $14(3-60)$ \\
\hline Ortiz [297] & 2000 & 103 & II, III & 0 & Prospektiv & 93 & k. A. & 8 & 12 \\
\hline Gustafson [162] & 2002 & 42 & II, III & 0 & Retrospektiv & 55 & k. A. & $\begin{array}{l}\text { Minor: } 31 \\
\text { major: } 11\end{array}$ & 12 \\
\hline Joy [203] & 2002 & 19 & II, III & $5(3 / 63)$ & $\begin{array}{l}\text { Retrospektiv } \\
\text { VG: } \bullet \text { Tab. } 5 \\
\text { und } 7\end{array}$ & 89 & k. A. & $\begin{array}{l}\text { Grad 1: } 50 \\
(7 / 13) \\
\text { Grad 2: } 21 \\
(3 / 13)\end{array}$ & $19(9-54)$ \\
\hline Köhler [219] & 2004 & 18 & $\begin{array}{l}\text { II (Huf- } \\
\text { eisen- } \\
\text { fistel) }\end{array}$ & 0 & $\begin{array}{l}\text { Prospektiv } \\
\text { VG: } \\
\text { - Tab. } 8,9 \\
\text { und } 11\end{array}$ & 61 & 35 & k. A. & $58(12-168)$ \\
\hline Perez [320] & 2006 & 27 & $\|, I I I$ & 0 & $\begin{array}{l}\text { Randomisiert }{ }^{\mathrm{a}} \\
\text { VG: } 0 \text { Tab. } 12\end{array}$ & 100 & 7,4 & 15 & $36(24-52)$ \\
\hline Uribe [442] & 2006 & 56 & $\begin{array}{l}\text { II, III, } \\
\text { RVF }\end{array}$ & 9 & Prospektiv & 92,9 & 7 & $\begin{array}{l}\text { Minor: } 12,5, \\
\text { major: } 9\end{array}$ & 44 \\
\hline $\begin{array}{l}\text { Van Koperen } \\
\text { [452] }\end{array}$ & 2008 & 54 & $\begin{array}{l}\text { II (dis- } \\
\text { tal) }\end{array}$ & 0 & $\begin{array}{l}\text { Retrospektiv } \\
\text { VG: Tab. } 4\end{array}$ & 77 & k. A. & k. A. & 13 \\
\hline Ortiz [299] & 2008 & 91 & II, III & k. A. & $\begin{array}{l}\text { Retrospektiv } \\
\text { VG: Tab. } 4\end{array}$ & k. A. & 18 & 12 & 42 \\
\hline Gustafsson [163] & 2006 & 83 & k. A. & 0 & Randomisiert ${ }^{\mathrm{b}}$ & $\begin{array}{l}57 \\
\text { Mit: } 62(26 / 42), \\
\text { ohne: } 51(21 / 41)\end{array}$ & 43 & k. A. & 12 \\
\hline Christoforidis [75] & 2009 & 43 & $\|$ & 0 & $\begin{array}{l}\text { Randomisiert }{ }^{c} \\
\text { VG: } 0 \text { Tab. } 17\end{array}$ & 73 & $11(3 / 27)$ & $\begin{array}{l}\text { Grad 0: } 48 \% \\
(11 / 23) \\
\text { Grad 2: } 35 \% \\
(8 / 23) \\
\text { Grad 3: } 17 \% \\
(4 / 23)\end{array}$ & 6 \\
\hline Adamina [11] & 2009 & 12 & ॥ & 0 & $\begin{array}{l}\text { Prospektiv } \\
\text { VG: } 0 \text { Tab. } 17\end{array}$ & 33 & k. A. & k. A. & k. A. \\
\hline Ortiz [298] & 2009 & 16 & $\begin{array}{l}\text { Il (dis- } \\
\text { tal) }\end{array}$ & 0 & $\begin{array}{l}\text { Randomisiert }^{d} \\
\text { VG: • Tab. } 17\end{array}$ & 87,5 & k. A. & k. A. & 12 \\
\hline Roig [349] & 2009 & 71 & II, III & 0 & $\begin{array}{l}\text { Retrospektiv } \\
\text { VG: } \bullet \text { Tab. } 7 \\
\text { und } 12\end{array}$ & 81,7 & k. A. & 43,6 & $13(12-60)$ \\
\hline Khafagy [208] & 2010 & 20 & II & 0 & $\begin{array}{l}\text { Randomisiert } \\
\text { VG: } \\
\text { Vab. } 9\end{array}$ & 95 & $\begin{array}{l}10 \text { (Gesamt- } \\
\text { heilung: } \\
85 \%)\end{array}$ & $10($ Grad 1) & k. A. \\
\hline A ba-bai-ke-re [1] & 2010 & 45 & I, II & 0 & $\begin{array}{l}\text { Randomisiert } \\
\text { VG: }{ }^{\mathrm{a}, \mathrm{b}} \\
\text { Tab. } 19\end{array}$ & 71 & k. A. & 9 & 6 \\
\hline Mushaya [278] & 2012 & 14 & $\begin{array}{l}\text { "Kom- } \\
\text { plex" }\end{array}$ & 0 & $\begin{array}{l}\text { Retrospektiv } \\
\text { VG: } \bullet \text { Tab. } 13\end{array}$ & $92(13 / 14)$ & k. A. & $\begin{array}{l}0 \text { (kein Unter- } \\
\text { schied) }\end{array}$ & 19 \\
\hline Stremitzer ${ }^{f}[412]$ & 2012 & 7 & II, III & 0 & $\begin{array}{l}\text { Fallstudie } \\
\text { VG: } \bullet T a b .9\end{array}$ & 86 & k. A. & k. A. & $85(26-136)$ \\
\hline $\operatorname{Tan}[420]$ & 2012 & 31 & II-III & 0 & $\begin{array}{l}\text { Retrospektiv } \\
\text { VG: } 0 \text { Tab. } 13\end{array}$ & 93,5 & k. A. & k. A. & $6(2-26)$ \\
\hline
\end{tabular}




\begin{tabular}{|c|c|c|c|c|c|c|c|c|c|}
\hline Autor & Jahr & $\begin{array}{l}\text { Anzahl } \\
\text { (n) }\end{array}$ & $\begin{array}{l}\text { Fistel- } \\
\text { typ }\end{array}$ & $\begin{array}{l}\text { Anteil } \\
\text { M. Crohn } \\
(\%)\end{array}$ & Studiendesign & Heilung (\%) & $\begin{array}{l}\text { Rezidive } \\
\text { (\%) }\end{array}$ & $\begin{array}{l}\text { Kontinenz- } \\
\text { störung (\%) }\end{array}$ & $\begin{array}{l}\text { Follow-up } \\
\text { (Monate) }\end{array}$ \\
\hline Goos [149] & 2015 & 36 & II, III & $36(13 / 36)$ & Retrospektiv & $\begin{array}{l}\text { Kryptoglandulär: } \\
83(19 / 23) \\
\text { Crohn: } 33(4 / 13)\end{array}$ & k. A. & $\begin{array}{l}\text { Kontinenz } \\
\text { gleich, Wex- } \\
\text { ner-Score } \\
\text { signifikant } \\
\text { besser }\end{array}$ & k. A. \\
\hline Uribe [441] & 2015 & $\begin{array}{l}137 \\
1: \\
78-m a l \\
\text { Exzision } \\
2: \\
41-\text { mal } \\
\text { Küretta- } \\
\text { ge der } \\
\text { Fistel }\end{array}$ & II, III & 0 & Retrospektiv & $\begin{array}{l}\text { Gesamt: } 94 \\
\text { Exzision: } 93,6 \\
\text { Kürettage: } 95,1\end{array}$ & k. A. & $\begin{array}{l}\text { Manometrie } \\
\text { schlechter } \\
\text { Wexner-Score } \\
\text { gleich } \\
\text { Kneifdruck } \\
\text { Gruppe 1 } \\
\text { signifikant } \\
\text { schlechter }\end{array}$ & $23(>12)$ \\
\hline \multicolumn{10}{|c|}{ 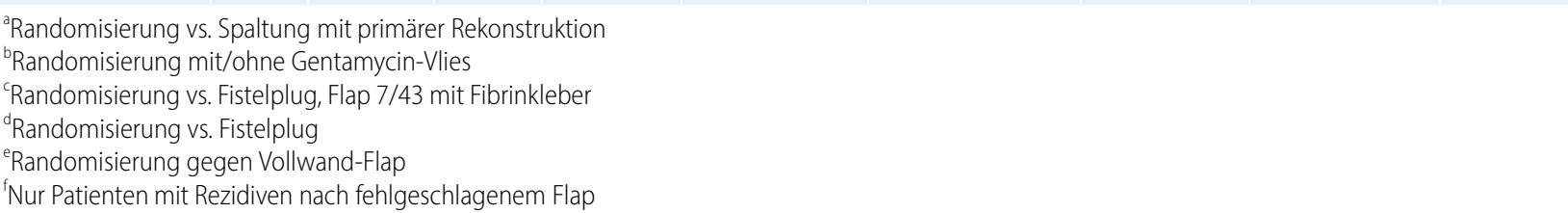 } \\
\hline
\end{tabular}

des Fistelverlaufs durch konventionelles Röntgen in $38 \%$, CT in $50 \%$ und MRT in $83 \%$. Letztere Untersuchung bestätigt somit den Stellenwert der MRT.

Mehrere Arbeiten vergleichen die klinische Untersuchung (in Narkose), Endosonographie und MRT und finden insbesondere bei Crohn-Patienten einehohe Übereinstimmung [52, 377]. Der positive Vorhersagewert für die Narkoseuntersuchung wurde von Buchanan et al. [52] jedoch mit lediglich $60 \%$ angegeben. Eine positive Beeinflussung des Operationsergebnisses ist in den Arbeiten von Ratto et al. [337] für die Endosonographie und Beets-Tan et al. [36] für die MRT beschrieben. Buchanan et al. [49] sehen nach Drainage auf dem Boden einer präoperativen MRT eine signifikant niedrigere Rezidivrate bei Rezidivfisteln (Rezidiv ohne MRT $57 \%$, mit MRT $16 \%$; Nachbeobachtungszeit 12 Monate).

Der Nachteil der CT besteht in der Strahlenbelastung sowie der schlechteren Weichteildarstellung gegenüber der MRT. In der klinischen Routine spielt diese Untersuchung trotz Einzelfalldarstellungen bei extrasphinktären Fisteln [241] deshalb keine Rolle.

Ein Review der Bildgebung bei Analfisteln von Zbar et al. [494] sieht die Notwendigkeit einer zusätzlichen apparativen Diagnostik bei der kleinen Pati- entengruppe mit komplexen Analfisteln. Eindeutige Vorteile für eine Technik können nicht angegeben werden. Er verweist aber auf die Notwendigkeit, diese Verfahren Spezialkliniken zur Behandlung komplexer Analfisteln vorzubehalten.

Neben anderen eher allgemeinen Reviews [366, 433] erschien 2012 ein interessantes Review von Siddiqui et al. [391]: Für die MRT-Untersuchung ergab sich aus den Studien eine Sensitivität von 0,87 (95\% CI 0,63-0,96) und eine Spezifität von 0,69 (95\% CI 0,51-0,82); aus den Endosonographie-Studien ergab sich eine Sensitivität von 0,87 (95\% CI 0,70-0,95) und eine Spezifität von 0,43 (95\% CI 0,21-0,59). Beklagt wird die deutliche Heterogenität zwischen den verschiedenen Studien. Als Schlussfolgerung erscheint eine gleiche Sensitivität beider Verfahren, während die MRT in Bezug auf die Spezifität besser abschneidet.

$\mathrm{Zu}$ all diesen Studien ist anzumerken, dass in hohem Maße auch Crohn-Fisteln erfasst wurden, so dass die Wertigkeit dieser Diagnostikmaßnahmen in Bezug auf die Menge der kryptoglandulären Fisteln kritisch gesehen werden muss.

Als neue Methode wurde 2016 von Sarici et al. [364] eine Diagnostik mittels Infrarot-Thermographie vorgestellt. Weitere Erkenntnisse hierzu liegen noch nicht vor.
Fazit für die Praxis. Weitergehende bildgebende Verfahren sollen bei komplexen und rezidivierenden Analfisteln in Erwägung gezogen werden und können in einigen Fällen relevante Zusatzinformationen bieten. Während die radiologische Fisteldarstellung mittels Kontrastmittel als obsolet anzusehen ist, sind Endosonographie und MRT weitgehend als gleichwertig anzusehen, wobei die technische Qualität der verwendeten Geräte eine große Rolle spielt und die Ultraschall-3D-Technik überlegen erscheint. Der Vorteil der Endosonographie liegt in der problemlosen und kostengünstigeren Anwendung, ist jedoch in hohem Maße von der Erfahrung des Untersuchers abhängig. Die MRT ist dagegen kostenintensiv, nicht immer verfügbar und in ihrer Aussagekraft von den technischen Voraussetzungen abhängig, jedoch bei analfernen Läsionen der Endosonographie vorzuziehen. Der Vorteil der MRT ist ferner eine schmerzfreie Bildakquisition, welche untersucherunabhängig ausgewertet werden kann. Eine Veränderung der Leitlinienempfehlungen durch neue Studien gegenüber der Erstfassung ergibt sich nicht.

- Evidenzlevel: 1a

- Empfehlungsgrad: A

- Konsensusstärke: starker Konsens 
Tab. 11 Ergebnisse des anodermalen Verschiebelappens (Anoderm-Flap)

\begin{tabular}{|c|c|c|c|c|c|c|c|c|c|}
\hline Autor & Jahr & $\begin{array}{l}\text { Anzahl } \\
\text { (n) }\end{array}$ & Fisteltyp & $\begin{array}{l}\text { Anteil } \\
\text { M. Crohn } \\
(\%)\end{array}$ & Studiendesign & Heilung (\%) & $\begin{array}{l}\text { Rezidive } \\
\text { (\%) }\end{array}$ & $\begin{array}{l}\text { Kontinenzstörung } \\
\text { (\%) }\end{array}$ & $\begin{array}{l}\text { Follow-up } \\
\text { (Monate) }\end{array}$ \\
\hline Köhler [216] & 1996 & 31 & II, III & 19 & Prospektiv & 93,5 & 6,8 & 16 & 12 \\
\hline Jun [204] & 1999 & 40 & Ilb, III & 0 & Retrospektiv & 95 & k. A. & 0 & $17(6-24)$ \\
\hline $\begin{array}{l}\text { Zimmerman } \\
\text { [502] }\end{array}$ & 2001 & 26 & II, (Rezidiv) & 0 & Retrospektiv & 46 & k. A. & 30 & 25 \\
\hline $\operatorname{Amin}^{b}[22]$ & 2003 & 18 & I, II, III & 0 & Retrospektiv & 83 & 13 & 0 & $19(3-60)$ \\
\hline $\begin{array}{l}\text { Sungurtekin }{ }^{\mathrm{b}} \\
\text { [416] }\end{array}$ & 2004 & 65 & II, III & 0 & Retrospektiv & 91 & k. A. & 0 & $32(12-52)$ \\
\hline Köhler [219] & 2004 & 8 & $\begin{array}{l}\text { II (Hufei- } \\
\text { senfistel) }\end{array}$ & 0 & $\begin{array}{l}\text { Prospektiv } \\
\text { VG: } \bullet \text { Tab. } 8\end{array}$ & 75 & 25 & k. A. & $58(12-168)$ \\
\hline Hossack [189] & 2005 & 16 & III (Rezidiv) & k. A. & Retrospektiv & 94 & k. A. & 70 (Verbesserung!) & $20(1,5-43)$ \\
\hline Ho [181] & 2005 & 10 & II & 0 & Randomisiert $^{c}$ & 70 & k. A. & k. A. & 9 \\
\hline Ellis [120] & 2006 & $\begin{array}{l}12 \\
11\end{array}$ & k. A. & 0 & $\begin{array}{l}\text { Randomisiert }^{d} \\
\text { VG: } 0 \text { Tab. } 10 \\
\text { und } 14\end{array}$ & $\begin{array}{l}75(A) \\
73(B)\end{array}$ & k. A. & k. A. & 22 \\
\hline Ellis [121] & 2007 & 25 & ॥ & 0 & $\begin{array}{l}\text { Retrospektiv } \\
\text { VG: } \bullet \text { Tab. } 9\end{array}$ & 80 & k. A. & k. A. & $10(6-22)$ \\
\hline \multicolumn{10}{|c|}{$\begin{array}{l}{ }^{\mathrm{a}} 52 \% \text { vorbestehende Kontinenzstörung } \\
{ }^{\mathrm{V} V} \text {-Y-Lappen }\end{array}$} \\
\hline
\end{tabular}

\section{Therapieverfahren}

Die Diagnose einer Analfistel stellt grundsätzlich eine Operationsindikation dar und dient der Vorbeugung eines rezidivierenden septischen Prozesses. Eine spontane Abheilung einer Analfistel ohne operative Intervention muss als extrem unwahrscheinlich angesehen werden. Eine - sehr seltene maligne Entartung von persistierenden Fisteln wird in der Literatur beschrieben (siehe entsprechender Absatz). Die Wahl des operativen Therapieverfahrens richtet sich nach dem Fistelverlauf und ihrem Verhältnis zum Analsphinkter. Zu unterscheiden ist dabei zwischen Verfahren mit bewusster Durchtrennung von Schließmuskelanteilen (Fistelspaltung) und solchen, die die Schließmuskelintegrität weitestgehend $\mathrm{zu}$ erhalten suchen (sog. plastischer Fistelverschluss). In neueren Verfahren werden Biomaterialien (Fibrin, Fistelplug) zur Okklusion des Fistelgangs verwendet.

Unter Berücksichtigung der oben dargestellten Methodik wurde die zugängliche Literatur ausgewertet und in Evidenztabellen erfasst, die im Anhang beigefügt sind. Die Einteilung des Fisteltyps wurde an die in $\bullet$ Abb. 1 dargestellte Klassifikati- on angeglichen, soweit dies möglich war. Analog zur Literatur wurde zwischen tiefen (distalen) und hohen (proximalen) transsphinktären Fisteln unterschieden. Bei einem Anteil unter $20 \%$ wurden auch Studien mit Einschluss von Crohn-Fisteln für die vorliegende Auswertung berücksichtigt. Andererseits machen viele Studien jedoch keine Angaben zur Genese der Fisteln, so dass hier, insbesondere in älteren Publikationen, möglicherweise auch Crohn-Fisteln eingeschlossen sind. Die Beurteilung der postoperativen Inkontinenzrate variiert sehr stark zwischen den Publikationen, da eine Definition dieses Begriffs fehlt. Erwartungsgemäß liegen die Zahlen umso höher, je diffiziler die jeweilige Kontinenzfunktion erfasst wurde. Am höchsten liegen die Daten bei Verwendung eines Inkontinenzscores.

Als Heilung wurde der definitive Verschluss des äußeren Fistelostiums und als Rezidiv das Wiederauftreten einer Fistel nach zwischenzeitlicher kompletter Abheilung angesehen. Leider wird in vielen Studien nicht klar zwischen diesen Varianten unterschieden, so dass hier versucht wurde, die entsprechenden Daten zuzuordnen. Heute sollte man Fisteln, die nie komplett heilen als persistent bezeich- nen und differenziert beurteilen. Die beschriebenen Rezidivraten in den Kurzzeitbeobachtungen wurden deshalb als Heilung erfasst, da i. d. R. nicht dargestellt wird, ob die Fistel zum Untersuchungszeitpunkt persistierte oder wieder aufgetreten war.

Einige Arbeiten berichten allgemein über das Krankengut der Klinik [249, 328, 349, 393]. Im Vergleich zwischen Allgemeinchirurgen und Kolorektalchirurgen konnten Nwaejike et al. [286] für letztere eine konservativere Behandlungsstrategie und weniger Rezidive nachweisen.

Eine besondere Risikogruppe stellen Rezidivfisteln dar. Hier bestimmen das Ausmaß der Voroperationen und die Komplexität der Fistel die Heilungswahrscheinlichkeit und Inkontinenzrate. Publizierte Ergebnisse mit größeren $\mathrm{Pa}$ tientenzahlen liegen nicht vor.

\section{Konservative Therapie}

Als konservativer Therapieansatz wurde von Attaallah et al. [30] die Anwendung von Silbernitrat-Spülungen beschrieben. Bei 56 Patienten sahen sie eine Heilungsrate von $52 \%$ bei einem Follow-up von 10 Monaten. Insgesamt wurden 1-10 (Median 4) Spülungen durchgeführt. 


\begin{tabular}{|c|c|c|c|c|c|c|c|c|c|}
\hline Autor & Jahr & $\begin{array}{l}\text { Anzahl } \\
(n)\end{array}$ & Fisteltyp & $\begin{array}{l}\text { Anteil } \\
\text { M. Crohn } \\
(\%)\end{array}$ & Studiendesign & $\begin{array}{l}\text { Heilung } \\
(\%)\end{array}$ & $\begin{array}{l}\text { Rezidive } \\
(\%)\end{array}$ & $\begin{array}{l}\text { Kontinenz- } \\
\text { störung (\%) }\end{array}$ & $\begin{array}{l}\text { Follow-up } \\
\text { (Monate) }\end{array}$ \\
\hline Parkash [311] & 1985 & 120 & k. A. & k. A. & Retrospektiv & 83,3 & $3(3 / 100)$ & 4 & $<60$ \\
\hline Christiansen [72] & 1995 & 14 & II, III, Rezidiv & 0 & Retrospektiv & 86 & k. A. & 21,4 & $12-48$ \\
\hline Gemsenjäger [141] & 1996 & 21 & I, II, III & k. A. & Retrospektiv & 95 & 5 & k. A. & $2-9$ \\
\hline Perez [319] & 2005 & 35 & II (distal), III, IV & k. A. & Retrospektiv & 94 & k. A. & 12,3 & 32 \\
\hline Perez [320] & 2006 & 28 & II (distal), III & 0 & $\begin{array}{l}\text { Randomsiert } \\
\text { VG: } 0 \text { Tab. } 9\end{array}$ & 100 & 7,1 & 18 & $36(24-52)$ \\
\hline Jordan [202] & 2009 & 60 & II-IV & 0 & $\begin{array}{l}\text { Retrospektiv } \\
\text { VG: } \bullet \text { Tab. } 4 \\
\text { und } 9\end{array}$ & 90 & k. A. & k. A. & $19 \pm 25$ \\
\hline Roig [349] & 2009 & 75 & II, III & 0 & $\begin{array}{l}\text { Retrospektiv } \\
\text { VG: } \bullet \text { Tab. } 7 \\
\text { und } 10\end{array}$ & 89,4 & k. A. & 21,3 & $12(12-60)$ \\
\hline Kraemer [220] & 2011 & 38 & II-IV & $3 / 38$ & Retrospektiv & 97 & k. A. & 5 & $16(9-24)$ \\
\hline Arroyo [23] & 2012 & 70 & II, III & 0 & Prospektiv & 91,5 & k. A. & 8,5 & $<12$ \\
\hline Ratto [340] & 2013 & 72 & „Komplex" & 0 & Prospektiv & 96 & k. A. & 12 (Soiling) & 29 \\
\hline Hirschburger [179] & 2014 & 50 & II & 0 & Retrospektiv & $\begin{array}{l}98 \text { (ge- } \\
\text { samt } 88)\end{array}$ & 10 & 6 & 22 \\
\hline
\end{tabular}

Ein kritischer Kommentar wurde hierzu von Doll et al. [106] verfasst, die im eigenen Krankengut nur bei $27 \%$ eine Heilung beobachteten.

Fazit für die Praxis. Auch neuere Studien konnten keine effektiven konservativen Therapieverfahren nachweisen.

- Evidenzlevel: 4

- Konsensusstärke: starker Konsens

\section{Fistelspaltung}

Das einfachste und am häufigsten angewandte Operationsverfahren $[45,112]$ stellt die Spaltung der Fistel, d.h. die Durchtrennung des zwischen Fistelgang und dem Analkanal gelegenen Gewebes, dar.

Hier werden 2 Verfahren unterschieden:

1. die Fistelspaltung mit reiner Durchtrennung des Gewebes zwischen dem Fistelgang und dem Anoderm,

2. die Fistulektomie mit radikaler Entfernung des Fistelgangs. Dieses Verfahren wurde zunächst von Parks beschrieben [314].

Die Ergebnisse aus der Literatur unterscheiden nicht immer klar zwischen diesen beiden Verfahren. Eine randomisier- te endosonographische Studie wies nach Fistulektomie deutlich größere Sphinkterdefekte nach [38].

Aus der Literatur konnten 34 Studien identifiziert werden, die Ergebnisse der Spaltungsmethode angeben. Sechs Arbeiten konnten gegenüber der Erstfassung hinzugefügt werden (• Tab. 4). In der Mehrzahl handelt es sich um retrospektive Beobachtungsstudien. Zu unterscheiden ist dabei zwischen 2 Gruppen:

1. Studien, die eine Spaltung bei allen

Fisteltypen (I-III) durchführten und somit lediglich heterogene Ergebnissen vorstellen.

2. Studien, die eine Spaltung lediglich bei oberflächlichen intersphinktären oder distalen transsphinktären Fisteln durchführten.

Die Heilungsraten in diesen Studien liegen zwischen 74 und $100 \%$. Die Inkontinenzraten differieren deutlich zwischen 0 und $45 \%$. Mylonakis et al. [279] beschreiben bei $38 \%$ der Operierten Kontinenzeinbußen geringeren Ausmaßes. Garcia-Aguilar et al. [134] beobachteten insgesamt eine Rate an Kontinenzstörungen von $45 \%$. In $\mathrm{Ab}$ hängigkeit vom Fisteltyp betrug diese $37 \%$ bei Typ I, $54 \%$ bei Typ II und $80 \%$ bei Typ III. Ein Anstieg des Kon- tinenzscores wird von Cavanaugh et al. [59] bei bis zu $64 \%$ der Operierten beobachtet. Dieses ist gleichzeitig mit einer Minderung der Lebensqualität assoziiert. Letztlich muss in diesem Zusammenhang jedoch festgehalten werden, dass die intraoperative Einstufung einer Fistel durch den Operateur als oberflächlich mit einem hohen Maß an Subjektivität verbunden ist.

Lediglich 6 Studien bieten eine randomisierte Gegenüberstellung verschiedener Techniken. In der ältesten randomisierten Studie vergleichen Ho et al. [183] die Fistelexzision mit und ohne Marsupialisation der Wundränder. Sie fanden eine schnellere Wundheilung nach Verkleinerung der Wundhöhle. Die Heilungsraten waren insgesamt gleich. In einer weiteren randomisierten Studie aus dem Jahr 2001 [182] vergleicht die gleiche Arbeitsgruppe die Fistelspaltung mit der Ayurvedafadeneinlage (Prinzip des schneidenden chemischen Fadens). Bei 100 \%iger Heilungsrate in beiden Gruppen war die Rate der Kontinenzstörungen in der Ayurvedagruppe etwas höher. Im Jahr 2002 randomisierten Lindsey et al. [238] die Spaltung gegen die Fibrinkleberapplikation. Während durch die Spaltung alle oberflächlichen Fisteln ohne nennenswerte Kontinenzeinbußen 


\section{Leitlinien}

Tab. 13 Ergebnisse der LIFT-Operation

\begin{tabular}{|c|c|c|c|c|c|c|c|c|c|}
\hline Autor & Jahr & $\begin{array}{l}\text { Anzahl } \\
(n)\end{array}$ & Fisteltyp & $\begin{array}{l}\text { Anteil } \\
\text { M. Crohn } \\
(\%)\end{array}$ & Studiendesign & $\begin{array}{l}\text { Heilung } \\
(\%)\end{array}$ & $\begin{array}{l}\text { Rezidive } \\
(\%)\end{array}$ & $\begin{array}{l}\text { Kontinenz- } \\
\text { störung (\%) }\end{array}$ & $\begin{array}{l}\text { Follow-up } \\
\text { (Monate) }\end{array}$ \\
\hline Matos [255] & 1993 & 13 & $\begin{array}{l}\text { Diverse Rezi- } \\
\text { divfisteln }\end{array}$ & k. A. & Retrospektiv & 54 & k. A. & 0 & k. A. \\
\hline $\begin{array}{l}\text { Rojanasakul } \\
\text { [352] }\end{array}$ & 2007 & 18 & II-III & k. A. & Prospektiv & 94,4 & k. A. & 0 & $<6,5$ \\
\hline Shanwani [386] & 2010 & 45 & II, „Komplex" & k. A. & Retrospektiv & 82 & k. A. & 0 & $9(2-16)$ \\
\hline Bleier [42] & 2010 & 39 & $\begin{array}{l}\text { II, III, RVF } \\
\text { (Rezidivfisteln: } \\
\text { 29) }\end{array}$ & k. A. & Retrospektiv & 57 & k. A. & 0 & 4,5 \\
\hline Ellis ${ }^{\mathrm{a}}[118]$ & 2010 & 31 & $\|$ & 13 & Retrospektiv & $94(29 / 91)$ & k. A. & 0 & $15(12-40)$ \\
\hline Ooi [295] & 2011 & 25 & $\begin{array}{l}\text { II (Rezidivfis- } \\
\text { teln: 12) }\end{array}$ & 0 & Retrospektiv & 68 & k. A. & 0 & $5,5(1-11)$ \\
\hline Sileri [394] & 2011 & 18 & II, III, RVF & 0 & Prospektiv & 83 & k. A. & k. A. & 6 \\
\hline Aboulian [8] & 2011 & 25 & II (hoch) & k. A. & Retrospektiv & 68 & k. A. & k. A. & $6(2-13)$ \\
\hline $\operatorname{Tan}[422]$ & 2011 & 93 & I, II, III & k. A. & Retrospektiv & $\begin{array}{l}92,5 \\
(86 / 93)\end{array}$ & $6,5(6 / 93)$ & k. A. & $\begin{array}{l}1,5 \\
(0,25-20)\end{array}$ \\
\hline Abcarian [4] & 2012 & 41 & II & 2,5 & Retrospektiv & $\begin{array}{l}74 \text { (Primär- } \\
\text { operation: } \\
90 \text { ) }\end{array}$ & k. A. & 0 & $\begin{array}{l}4,5 \\
(0,5-16)\end{array}$ \\
\hline $\operatorname{Han}^{b}[171]$ & 2011 & 21 & ॥ & k. A. & Retrospektiv & 95 & k. A. & $5($ Grad 1) & $14(12-15)$ \\
\hline Mushaya [278] & 2012 & 25 & „Komplex" & 0 & $\begin{array}{l}\text { Retrospektiv } \\
\text { VG: - Tab. } 10\end{array}$ & $92(2 / 25)$ & k. A. & 0 & 19 \\
\hline $\operatorname{Tan}[420]$ & 2012 & 24 & II-III & 0 & $\begin{array}{l}\text { Retrospektiv } \\
\text { VG: } \bullet \text { Tab. } 10\end{array}$ & 62,5 & k. A. & k. A. & $13(4-67)$ \\
\hline $\begin{array}{l}\text { Van Onkelen } \\
{[455]}\end{array}$ & 2012 & 22 & Ila & 0 & Prospektiv & 82 & k. A. & 0 & 6 \\
\hline $\begin{array}{l}\text { Van Onkelen }{ }^{c} \\
{[454]}\end{array}$ & 2012 & 41 & ॥ & 0 & Retrospektiv & $\begin{array}{l}51 \text { (21/41) } \\
71 \text { (nach } \\
\text { Spaltung) } \\
\text { 8-mal in- } \\
\text { tersphink- } \\
\text { täre Fistel } \\
\text { gespalten }\end{array}$ & k. A. & 0 & $15(7-21)$ \\
\hline Wallin [469] & 2012 & 93 & $\begin{array}{l}\text { II (Rezidivfis- } \\
\text { teln: 32) }\end{array}$ & 0 & Retrospektiv & 40 & k. A. & $\begin{array}{l}25(\text { Grad 1) } \\
6(\operatorname{Grad} 2)\end{array}$ & 19 \\
\hline Lo [240] & 2012 & 25 & II, III & 0 & Retrospektiv & 89 & k. A. & k. A. & $10(1-22)$ \\
\hline Chen [66] & 2012 & 10 & ॥ & k. A. & Prospektiv & 100 & 20 & 0 & $7(6-10)$ \\
\hline $\begin{array}{l}\text { Sirikurnpiboon } \\
\text { [399] }\end{array}$ & 2013 & 41 & II & 0 & Retrospektiv & $\begin{array}{l}83 \\
81 \text { (nur } \\
\text { LIFT) } \\
85 \text { (LIFT u. } \\
\text { Kürettage) }\end{array}$ & k. A. & 0 & 5 \\
\hline Liu [239] & 2013 & 38 & II & k. A. & Retrospektiv & $68(60)$ & 8 & 0 & $\begin{array}{l}26(3-44) \\
68 \%>12\end{array}$ \\
\hline Chew [67] & 2013 & $\begin{array}{l}29 \\
\text { (LIFT) } \\
5 \text { (Bio- } \\
\text { LIFT) }\end{array}$ & $\begin{array}{l}\text { II, anovaginal, } \\
\text { Hufeneisen }\end{array}$ & 0 & Prospektiv & $63(60)$ & 3 & 0 & $5(1,5-8)$ \\
\hline Tsunoda $^{d}$ [439] & 2013 & 20 & II, „komplex“ & 0 & Prospektiv & 95 & k. A. & 0 & $18(3-32)$ \\
\hline
\end{tabular}




\begin{tabular}{|c|c|c|c|c|c|c|c|c|c|}
\hline Autor & Jahr & $\begin{array}{l}\text { Anzahl } \\
(n)\end{array}$ & Fisteltyp & $\begin{array}{l}\text { Anteil } \\
\text { M. Crohn } \\
\text { (\%) }\end{array}$ & Studiendesign & $\begin{array}{l}\text { Heilung } \\
(\%)\end{array}$ & $\begin{array}{l}\text { Rezidive } \\
(\%)\end{array}$ & $\begin{array}{l}\text { Kontinenz- } \\
\text { störung (\%) }\end{array}$ & $\begin{array}{l}\text { Follow-up } \\
\text { (Monate) }\end{array}$ \\
\hline Lehmann [230] & 2013 & 17 & $\begin{array}{l}\text { II, III, RVF (nur } \\
\text { Rezidivfisteln) }\end{array}$ & k. A. & Prospektiv & $\begin{array}{l}65(1 \mathrm{Mo}- \\
\text { nat) } \\
47 \\
(13,5 \text { Mo- } \\
\text { nate) }\end{array}$ & 12 & 0 & $\begin{array}{l}4(1-6) \\
\text { LZ: } 13,5\end{array}$ \\
\hline Campbell [58] & 2013 & 20 & $\begin{array}{l}\text { k. A. } \\
\text { (9-mal Rezi- } \\
\text { div) }\end{array}$ & 0 & Retrospektiv & $\begin{array}{l}70 \text { (1 Mo- } \\
\text { nat) } \\
80 \text { (3 Mo- } \\
\text { nat) }\end{array}$ & k. A. & 0 & 3 \\
\hline Hall [165] & 2014 & 43 & I, II, RVF & 12 & $\begin{array}{l}\text { Prospektiv, } \\
\text { multizentrisch } \\
\text { (16 Operateure) } \\
\text { VC: } 0 \text { Tab. } 4,9 \\
\text { und } 17\end{array}$ & 79 & k. A. & $\begin{array}{l}\text { CCFI postop. } \\
\text { niedriger }\end{array}$ & 3 \\
\hline $\operatorname{Tan}^{\mathrm{a}}[421]$ & 2014 & $\begin{array}{l}13(16 \\
\text { Fisteln })\end{array}$ & II & k. A. & Retrospektiv & 69 & k. A. & 0 & $26(12-51)$ \\
\hline Tomiyoshi [432] & 2014 & 8 & II & 0 & Fallstudie & 75 & k. A. & 12,5 & 1 \\
\hline Madbouly [247] & 2014 & 35 & ॥ & 0 & $\begin{array}{l}\text { Randomisiert } \\
\text { VG: } \\
\text { VTab. } 9\end{array}$ & 94 & $74(26 / 35)$ & 0 (4 Wochen) & 12 \\
\hline Sileri [396] & 2014 & 26 & I, II, RVF & k. A. & Retrospektiv & 73 & k. A. & 0 & $>16$ \\
\hline Schulze [370] & 2015 & 75 & „Komplex" & 0 & Prospektiv & 88 & k. A. & 1,3 & 15 \\
\hline \multicolumn{10}{|c|}{$\begin{array}{l}\text { 'Bio-LIFT-Verfahren } \\
\text { 'Zusätzlicher Fistelplug } \\
\text { 'Zusätzlicher Mukosa-Flap } \\
\text { dLigatur, plus schneidende Fadendrainage des Externusanteils } \\
\text { 'Randomisiert gegen Mukosa-Flap, postoperative Schmerzen r }\end{array}$} \\
\hline
\end{tabular}

geheilt werden konnten, lag die Heilungsrate bei den einfachen Fisteln nach Fibrinkleberapplikation bei lediglich $33 \%$ und bei den höher gelegenen Fisteln bei $46 \%$. Die Studie bietet jedoch ein maximales Follow-up von lediglich 3 Monaten. Die derzeit aktuellste randomisierte Studie stammt aus dem Jahr 2006 [322]. Auch hier wird die Fistelspaltung mit und ohne Marsupialisation verglichen. Insgesamt liegt ein sehr inhomogenes Patientengut mit Einschluss von oberflächlichen bis extrasphinktären, zum Teil rezidivierten Fisteln vor. Über Kontinenzstörungen und Rezidivraten wird keine Aussage gemacht. Die Heilungsrate liegt bei $86 \%$ mit und $89 \%$ ohne Marsupialisation. Auch die Arbeit von Jain et al. [196] vergleicht die Fistulektomie mit und ohne Marsupialisation bei tiefen Fisteln. Die Heilung betrug in beiden Gruppen 100 \%, wobei die Heilungsdauer nach Marsupialisation eher länger war. Das Follow-up war mit 3 Monaten sehr kurz, so dass es nicht verwunderlich ist, dass keine ne- gative Beeinträchtigung der Kontinenz beobachtet wurde.

Dutta et al. [110] randomisierten die Fistulektomie gegen den Ayurvedafaden als schneidenden Faden. Insgesamt schnitten beide Verfahren weitgehend gleich ab, wobei die Rate der Kontinenzstörungen mit $12 \%$ in der Fistulektomiegruppe höher war als in der Vergleichsgruppe (4\%). Die Wundheilungsdauer war nach Fadeneinlage länger. Trotzdem war in dieser Gruppe die Arbeitsunfähigkeit kürzer.

Interessant ist eine aktuelle Arbeit mit multizentrischen Ergebnissen bei $537 \mathrm{~Pa}$ tienten [150]. Bei einem Follow-up von mehr als 60 Monaten betrug die primäre Heilungsrate 84 \%. Bemerkenswert ist die beschriebene hohe Rate an Kontinenzstörungen mit $74 \%$ (Major-Inkontinenz: $28 \%$ ), wobei sich die Lebensqualität nicht von der der Allgemeinbevölkerung unterschied.

Eine Aufarbeitung historischer Krankenunterlagen von Blumetti et al. [45] zeigte eine deutliche Abkehr von Spal- tungsverfahren hin zu sphinkterschonenden Verfahren.

Fazit für die Praxis. Die Freilegung oder Spaltung einer Analfistel stellt die am häufigsten angewandte Therapiemaßnahme dar. Bei oberflächlichen Fisteln kann dabei eine fast 100 \%ige Heilungsrate erzielt werden. Die postoperativen Inkontinenzraten werden in der Literatur meist als relativ niedrig beschrieben, stellen jedoch eine ernstzunehmende Folgeerscheinung dar. In jedem Fall steigt die Inkontinenzrate mit der Masse an durchtrenntem Sphinkteranteil. Die derzeit vorliegenden randomisierten Studien erlauben keine klare Aussage. Eine großzügige Spaltung sollte in jedem Fall vermieden werden, zumal dem Kontinenzerhalt im Behandlungsalgorithmus eine zunehmende Bedeutung zukommt. - Evidenzlevel: 2b

- Empfehlungsgrad: B

- Konsensusstärke: starker Konsens 


\section{Leitlinien}

Tab. 14 Ergebnisse der Fistelkürettage und Fibrinkleber-Instillation

\begin{tabular}{|c|c|c|c|c|c|c|c|c|c|}
\hline Autor & Jahr & $\begin{array}{l}\text { Anzahl } \\
(n)\end{array}$ & Fisteltyp & $\begin{array}{l}\text { Anteil } \\
\text { M. Crohn } \\
(\%)\end{array}$ & Studiendesign & Heilung (\%) & $\begin{array}{l}\text { Rezidiv } \\
\text { (\%) }\end{array}$ & $\begin{array}{l}\text { Kontinenz- } \\
\text { störung (\%) }\end{array}$ & $\begin{array}{l}\text { Follow-up } \\
\text { (Monate) }\end{array}$ \\
\hline Hjortrup $^{\mathrm{a}}[180]$ & 1991 & 23 & k. A. & 0 & Prospektiv & 52 & k. A. & k. A. & $12-26$ \\
\hline $\begin{array}{l}\text { Venkatesch } \\
\text { [464] }\end{array}$ & 1999 & 12 & $\begin{array}{l}\text { I, II, III, } \\
\text { Rezidiv }\end{array}$ & 0 & Prospektiv & $\begin{array}{l}75 \\
\text { Kryptoglandulär: 80, } \\
\text { M. Crohn: } 0\end{array}$ & k. A. & k. A. & $26(9-57)$ \\
\hline Cintron [79] & 1999 & 26 & I, II, III & $8(2 / 26)$ & Prospektiv & 81 & k. A. & k. A. & 3,5 \\
\hline Cintron [80] & 2000 & 76 & I-IV & $16(12 / 76)$ & Prospektiv & 61 (I: 82, II: 62) & k. A. & k. A. & 12 \\
\hline Park [310] & 2000 & 22 & I, II, III & 0 & Prospektiv & I: 75, II: 80, III: 67 & k. A. & k. A. & 6 \\
\hline Patrlj [315] & 2000 & 69 & I, II & 0 & Retrospektiv & 74 (I: 77; II: 70) & k. A. & k. A. & $28(18-36)$ \\
\hline Salim b [360] & 2001 & 6 & $\|$ & 0 & Prospektiv & 100 & k. A. & k. A. & $<3$ \\
\hline Lindsey [238] & 2002 & 19 & I, II, III & Ja & $\begin{array}{l}\text { Randomisiert }{ }^{\mathrm{c}} \\
\text { VG: • Tab. } 4\end{array}$ & $\begin{array}{l}\text { Einfache Fistel: } 33 \text { (2/6) } \\
\text { Komplexe Fistel: } 46 \\
(9 / 13)\end{array}$ & k. A. & 0 & 3 \\
\hline Greenberg [156] & 2002 & 15 & I, II, III & k. A. & Retrospektiv & 73 (I: 50, II: 78, III: 100) & k. A. & 0 & 4 \\
\hline $\operatorname{Chan}^{d}[61]$ & 2002 & 10 & $\begin{array}{l}\text { Subkutan, } \\
\text { I, II }\end{array}$ & 0 & Prospektiv & $\begin{array}{l}0: 100(3 / 3) \\
\mathrm{I}: 60(3 / 5) \\
\text { II: } 0(0 / 2)\end{array}$ & k. A. & k. A. & 7 \\
\hline Buchanan [50] & 2003 & 21 & II, III, IV & 0 & Prospektiv & 14 & k. A. & k. A. & 14 \\
\hline Sentovich [381] & 2003 & 48 & $\begin{array}{l}\text { Einfach/ } \\
\text { komplex }\end{array}$ & 10 & Prospektiv & 60 & $10(3 / 29)$ & 0 & $22(6-48)$ \\
\hline Zmora $^{e}[503]$ & 2003 & 37 & II, III & 19 & $\begin{array}{l}\text { Retrospektiv } \\
\text { VG: } \bullet \text { Tab. } 9\end{array}$ & $\begin{array}{l}41 \text { (Fibrin: 33, mit Flap: } \\
\text { 54) }\end{array}$ & k. A. & k. A. & 12 \\
\hline Tinay [429] & 2003 & 15 & k. A. & k. A. & Prospektiv & 73 & k. A. & 0 & 8 \\
\hline Jurczak [205] & 2004 & 31 & II, III & k. A. & Retrospektiv & 75 & k. A. & 0 & 9 \\
\hline Gisbertz [145] & 2005 & 27 & $\begin{array}{l}\text { I, II (proxi- } \\
\text { mal) }\end{array}$ & 0 & Retrospektiv & 33 & k. A. & 0 & 6 \\
\hline Singer ${ }^{9}[397]$ & 2005 & 75 & k. A. & $12,5(2 / 24)$ & Retrospektiv & $A: 21 ; B: 40 ; 31$ & k. A. & 0 & 27 \\
\hline Maralcan [251] & 2006 & 36 & I, II, III & 0 & Prospektiv & 78 (I: 44, II, 91, III: 75) & k. A. & k. A. & 12 \\
\hline $\begin{array}{l}\text { De la Portilla }{ }^{9} \\
\text { [95] }\end{array}$ & 2006 & 14 & II (distal) & 0 & Retrospektiv & 50 & k. A. & k. A. & 14 \\
\hline Barillari ${ }^{\mathrm{h}}[32]$ & 2006 & 21 & I, II, III & 0 & Retrospektiv & $\begin{array}{l}\text { Einfache Fistel: } 71 \text { (5/7) } \\
\text { Komplexe Fistel: } 71 \\
(10 / 14)\end{array}$ & 0 & k. A. & 18 \\
\hline Johnson [199] & 2006 & 10 & ॥ & 0 & $\begin{array}{l}\text { Randomisiert } \\
\text { VG: • Tab. } 17\end{array}$ & 40 & k. A. & k. A. & 3,5 \\
\hline Ellis [120] & 2006 & 28 & k. A. & 0 & $\begin{array}{l}\text { Randomsiert } \\
\text { VG: } \bullet \text { Tab. } 9 \\
\text { und } 11\end{array}$ & 54 & k. A. & k. A. & 22 \\
\hline Witte [485] & 2007 & 34 & $\begin{array}{l}\text { Subkutan, } \\
\text { I-IV }\end{array}$ & Ja & k. A. & $\begin{array}{l}\text { Einfache Fistel: } 56 \\
\text { Komplexe Fistel: } 54\end{array}$ & $\begin{array}{l}\text { k. A. } \\
\text { (2-mal } \\
\text { Rezi- } \\
\text { divab- } \\
\text { szess) }\end{array}$ & k. A. & 7 \\
\hline Tyler [440] & 2007 & 89 & II, III & 0 & Retrospektiv & 55 & k. A. & k. A. & k. A. \\
\hline Adams [14] & 2008 & 36 & II & 0 & Retrospektiv & 66 & 6 & k. A. & 6 \\
\hline Jain $^{h}$ [197] & 2008 & 20 & k. A. & k. A. & Retrospektiv & 85 & k. A. & k. A. & 6 \\
\hline $\begin{array}{l}\text { Van Koperen } \\
\text { [453] }\end{array}$ & 2008 & 26 & II (distal) & 0 & Retrospektiv & 44 & k. A. & k. A. & 13 \\
\hline Chung [77] & 2009 & 23 & II, III & 0 & $\begin{array}{l}\text { Prospektiv } \\
\text { VG: } 0 \text { Tab. } 5,9 \\
\text { und } 17\end{array}$ & 39 & k. A. & k. A. & 3 \\
\hline De Parades [98] & 2009 & 29 & I, II, III, RVF & 0 & Retrospektiv & 56 & 15 & k. A. & 12 \\
\hline
\end{tabular}




\begin{tabular}{|c|c|c|c|c|c|c|c|c|c|}
\hline Autor & Jahr & $\begin{array}{l}\text { Anzahl } \\
(n)\end{array}$ & Fisteltyp & $\begin{array}{l}\text { Anteil } \\
\text { M. Crohn } \\
(\%)\end{array}$ & Studiendesign & Heilung (\%) & $\begin{array}{l}\text { Rezidiv } \\
(\%)\end{array}$ & $\begin{array}{l}\text { Kontinenz- } \\
\text { störung (\%) }\end{array}$ & $\begin{array}{l}\text { Follow-up } \\
\text { (Monate) }\end{array}$ \\
\hline $\begin{array}{l}\text { Garcia-Olmo } \\
\text { [136] }\end{array}$ & 2009 & 25 & II, III, RVF & 29 & $\begin{array}{l}\text { Randomisiert }^{\mathrm{b}} \text {, } \\
\text { multizentrisch } \\
\text { VG } \bullet \text { Tab. } 16\end{array}$ & $16(4 / 25)$ & k. A. & k. A. & 38 \\
\hline Altomare [20] & 2010 & 38 & ॥ & 0 & $\begin{array}{l}\text { Randomisiert }^{\mathrm{k}} \\
\text { VG: }- \text { Tab. } 7\end{array}$ & 39 & k. A. & 0 & 12 \\
\hline $\begin{array}{l}\text { Queralto12 } \\
\text { [333] }\end{array}$ & 2010 & 34 & II, III & 0 & Retrospektiv & 68 & k. A. & 0 & $34(21-43)$ \\
\hline Yeung [488] & 2010 & 40 & $\begin{array}{l}\text { I, II, III, RVF, } \\
\text { Pouch-Vag. }\end{array}$ & $7,5(3 / 40)$ & Retrospektiv & $\begin{array}{l}\text { M. Crohn: } 0 \\
\text { Hohe Fistel: } 28,6(8 / 28) \\
\text { Einfache Fistel: } 41,7 \\
(5 / 12)\end{array}$ & k. A. & k. A. & $5,2(1-16)$ \\
\hline Panidis [306] & 2010 & 1 & $\begin{array}{l}\text { Hufeisen- } \\
\text { fistel }\end{array}$ & 0 & Fallstudie & 100 & 0 & 0 & 24 \\
\hline $\begin{array}{l}\text { Papavramidis } \\
\text { [308] }\end{array}$ & 2010 & 1 & III (HIV) & 0 & Fallstudie & 100 & k. A. & k. A. & 15 \\
\hline $\begin{array}{l}\text { Van der Hagen } \\
\text { [447] }\end{array}$ & 2011 & 15 & II, III & 0 & $\begin{array}{l}\text { Randomisiert } \\
\text { VG: } \bullet T a b .9\end{array}$ & 40 & k. A. & 0 & 49 \\
\hline De Oca ${ }^{m}$ [97] & 2011 & 28 & II & 0 & Prospektiv & 68 & k. A. & 0 & $20(3-60)$ \\
\hline Herreros [176] & 2012 & 56 & II, III & 0 & $\begin{array}{l}\text { Randomisiert }^{\circ} \\
\text { VG } \bullet \text { Tab. } 16\end{array}$ & 37 & k. A. & k. A. & 12 \\
\hline Maralcan [252] & 2011 & 46 & II, III & 0 & Prospektiv & $\begin{array}{l}87 \\
\text { Langzeit: } 63 \text { (nach } \\
\text { mehrfachen Injektio- } \\
\text { nen) }\end{array}$ & 37 & 0 & 54 \\
\hline \multicolumn{10}{|c|}{ 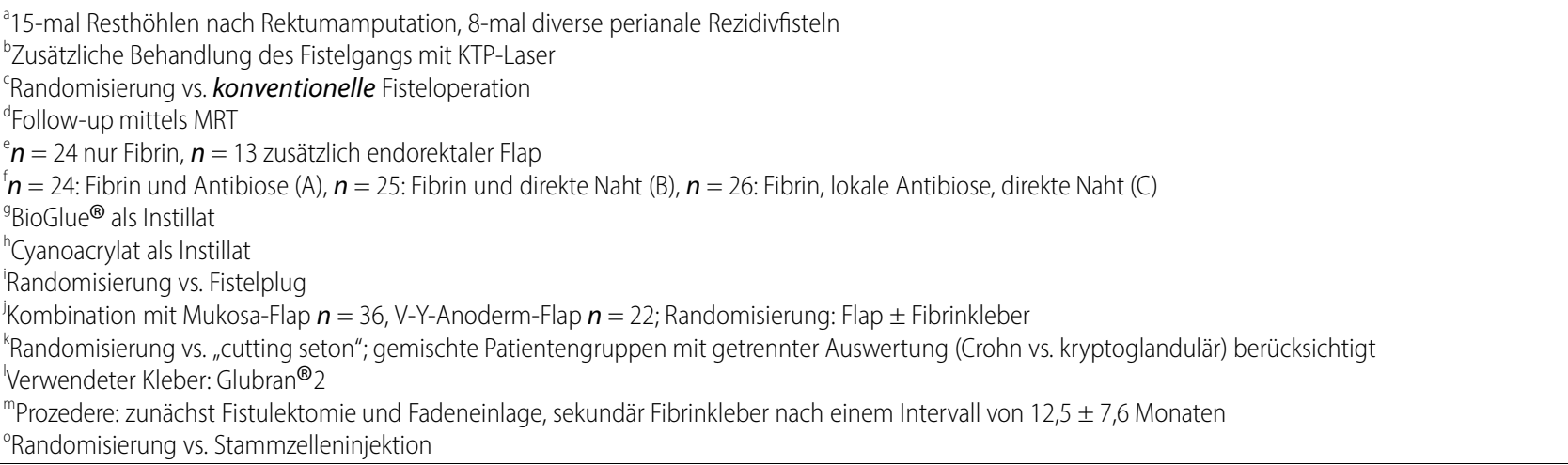 } \\
\hline
\end{tabular}

\section{Fadendrainage}

Die Anlage einer Fadendrainage zählt ebenfalls $\mathrm{zu}$ den häufig angewandten Verfahren in der Analfistelchirurgie. Als Material kommt entweder ein kräftiger geflochtener nichtresorbierbarer Faden (z. B. Seide) oder ein Kunststoffzügel („Vessel-Loop“) in Frage. Verschiedene Fadentypen wurden in der Literatur vorgestellt [263, 324, 344, 497].

Grundsätzlich ist zwischen 3 Verfahren zu unterscheiden:

1. Der lockere Faden als Markierung und Drainage einer Fistel im Rahmen der Abszesssanierung vor weiteren geplanten Maßnahmen

2. Der Faden als Langzeitdrainage mit dem Ziel der Schaffung eines stabilen Fistelkanals (fibrosierender Faden) vor weiteren Therapieoptionen oder als Dauerlösung

3. Der schneidende Faden mit dem Ziel der kontrollierten Durchtrennung des Schließmuskels

Faden als Drainage („loose seton“) Ziel dieses Verfahrens ist eine Langzeitdrainage der Abszesshöhle zur Verhinderung des frühzeitigen Verschlusses der äußeren Fistelöffnung. Später wird der Faden entfernt, um eine spontane Abheilung der Fistel zu ermöglichen. Von einigen Autoren wird dieses Verfahren mit einer primären Fistelexzision oder einer Internussphinkterotomie kombiniert [48, 134, 203, 233, 314, 484]. Ergebnisse der Fadendrainage als alleinige Therapie werden in 17 Publikationen (• Tab.5) vorgestellt, von denen 2 gegenüber der 1. Leitlinienversion neu sind. Die Heilungsrate differiert zwischen 33 und $100 \%$. Kontinenzstörungen werden mit einer Häufigkeit von 0 bis $62 \%$ angegeben. Vom Design stellen alle Arbeiten retrospektive Beobachtungsstudien dar. Randomisier- 
Tab. 15 Ergebnisse der Kollageninjektion (Permacol ${ }^{\circledR}$ )

\begin{tabular}{|c|c|c|c|c|c|c|c|c|c|}
\hline Autor & Jahr & $\begin{array}{l}\text { Anzahl } \\
(n)\end{array}$ & Fisteltyp & $\begin{array}{l}\text { Anteil } \\
\text { M. Crohn } \\
(\%)\end{array}$ & Studiendesign & Heilung (\%) & Rezidive (\%) & $\begin{array}{l}\text { Kontinenz- } \\
\text { störung (\%) }\end{array}$ & $\begin{array}{l}\text { Follow-up } \\
\text { (Monate) }\end{array}$ \\
\hline Hammond $^{\mathrm{a}}[170]$ & 2010 & $\begin{array}{l}\text { A: } 13 \\
\text { B: } 16\end{array}$ & II, III & k. A. & Prospektiv & $\begin{array}{l}54(7 / 13) \\
87(13 / 15)\end{array}$ & k. A. & 0 & 29 \\
\hline Sileri $^{\mathrm{b}}$ [395] & 2011 & 11 & II, III; RVF & 45 & Retrospektiv & 91 & k. A. & 0 & $6-15$ \\
\hline Giordano $^{c}[144]$ & 2016 & 28 & I, II & 0 & $\begin{array}{l}\text { Prospektiv, } \\
\text { multizentrisch }\end{array}$ & $\begin{array}{l}54(15 / 28) \\
\text { Typ I: } 67 \\
\text { Typ II: } 44\end{array}$ & k. A. & 3 & 12 \\
\hline
\end{tabular}

Tab. 16 Ergebnisse der Instillation von autologen Stammzellen

\begin{tabular}{|c|c|c|c|c|c|c|c|c|c|}
\hline Autor & Jahr & $\begin{array}{l}\text { Anzahl } \\
(n)\end{array}$ & Fisteltyp & $\begin{array}{l}\text { Anteil } \\
\text { M. Crohn } \\
(\%)\end{array}$ & Studiendesign & Heilung (\%) & Rezidive (\%) & $\begin{array}{l}\text { Kontinenz- } \\
\text { störung (\%) }\end{array}$ & $\begin{array}{l}\text { Follow-up } \\
\text { (Monate) }\end{array}$ \\
\hline Garcia-Olmo a [136] & 2009 & $\begin{array}{l}24 \\
25\end{array}$ & II, III, RVF & 29 & $\begin{array}{l}\text { Randomisiert }^{\mathrm{b}} \text {, } \\
\text { multizentrisch } \\
\text { VG: - Tab. } 14\end{array}$ & $\begin{array}{l}71(17 / 24) \\
16(4 / 25)\end{array}$ & k. A. & k. A. & 38 \\
\hline Van der Hagen ${ }^{\mathrm{b}}$ [446] & 2011 & 10 & II, III, IV & 0 & Retrospektiv & 90 & k. A. & 0 & $26(17-32)$ \\
\hline 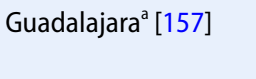 & 2012 & $\begin{array}{l}21 \\
13\end{array}$ & II, III, RVF & 24 & Retrospektiv & $\begin{array}{l}38(8 / 21) \\
15(2 / 13)\end{array}$ & k. A. & k. A. & $>36$ \\
\hline Herreros $^{\mathrm{a}}[176]$ & 2012 & $\begin{array}{l}A: 64 \\
B: 60 \\
C: 56\end{array}$ & II, III & 0 & $\begin{array}{l}\text { Randomisiert } \\
\text { VG: } \bullet \text { Tab. } 14\end{array}$ & $\begin{array}{l}39 \text { (1. Jahr: 57) } \\
43 \text { (1. Jahr: 52) } \\
37 \text { (1. Jahr: 37) }\end{array}$ & k. A. & k. A. & 12 \\
\hline De la Portilla ${ }^{a}$ [93] & 2013 & 24 & I, II, III, IV & 0 & Prospektiv & $\begin{array}{l}56 \text { (einzelne } \\
\text { Fisteln zu) } \\
30 \% \text { (alle } \\
\text { Fisteln zu) }\end{array}$ & k. A. & k. A. & 6 \\
\hline Göttgens $^{\mathrm{d}, \mathrm{e}}[153]$ & 2014 & 25 & ॥ & 0 & Retrospektiv & $81(17 / 21)$ & k.A & k. A. & 27 \\
\hline Kucharczyk ${ }^{f}[221]$ & 2014 & 2 & I, II & 0 & Fallstudie & 50 & k. A. & k. A. & 2 bzw. 6 \\
\hline \multicolumn{10}{|c|}{ 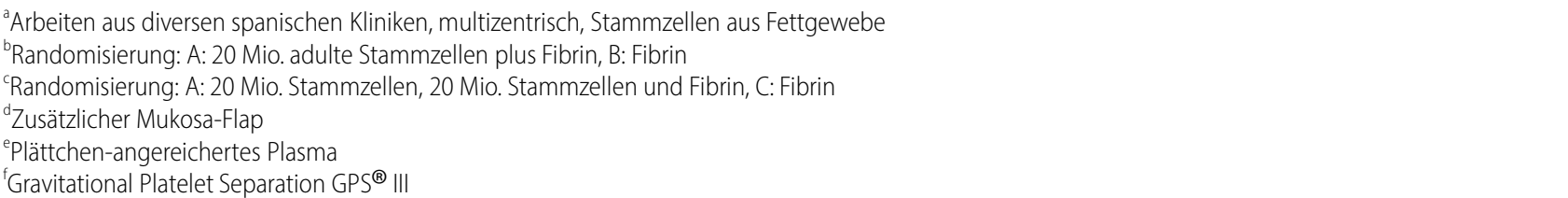 } \\
\hline
\end{tabular}

te Studien liegen nicht vor. Bezüglich der Fisteltypen beschreiben alle Publikationen ein gemischtes Patientengut. In einigen Fällen werden vor Entfernung des Fadens noch kleinere operative Interventionen vorgenommen, die nicht immer im Einzelnen auswertbar sind.

Zwei Publikationen [48, 233] übermitteln die Ergebnisse bei oberflächlichen Fisteln mit einer Heilungsrate von 64 bzw. $92 \%$ bei niedriger Inkontinenzrate. Die Ergebnisse bei höheren transund suprasphinktären Fisteln beschreiben Heilungsraten zwischen 33 und $90 \%$ bei einer medianen Inkontinenzrate von $39 \%$, wobei die hohe Inkontinenzrate nur durch begleitende Eingriffe und nicht durch die Fadendrainage an sich zu erklären ist.

Die interessanteste Arbeit aus dem Jahr 2004 [53], die jedoch leider einen relativ hohen Anteil von Crohn-Patienten berücksichtigt, zeigt im Langzeitverlauf das Wiederauftreten der Fisteln nach Entfernung des Fadens. So waren nach 5 Jahren $75 \%$ der Fisteln wieder offen, was die Bedeutung und Notwendigkeit der Langzeitbeobachtung hervorhebt.

Eine definitive Heilung von kryptoglandulären Analfisteln durch passagere Fadeneinlage dürfte nur in seltenen Fällen zu erzielen sein. Dies wird durch die Erfahrung der Autoren stark gestützt. In der Regel ist ein weiterer Eingriff erfor- derlich. Insofern stellt das beschriebene Patientengut eine sehr inhomogene und somit nur bedingt auswertbare Gruppe dar.

Die beiden neuen Publikationen fügen dem vorhandenen Wissen keine neuen Aspekte hinzu.

\section{Fibrosierender Faden}

Die Anlage eines fibrosierenden Fadens erfolgt i. d. R. entweder primär oder auch sekundär im Rahmen der Behandlung einer akuten oder persistierenden Entzündung. Nach Abheilung des entzündlichen Prozesses kommt es durch den liegenden Faden zu einer sukzessiven Fibrosierung des Fistelgangs vor weiteren 
Tab. 17 Ergebnisse von Fistelkürettage und Surgisis ${ }^{\Theta_{-}}$-Analfistelplug

\begin{tabular}{|c|c|c|c|c|c|c|c|c|c|}
\hline Autor & Jahr & $\begin{array}{l}\text { Anzahl } \\
\text { (n) }\end{array}$ & Fisteltyp & $\begin{array}{l}\text { Anteil } \\
\text { M. Crohn } \\
(\%)\end{array}$ & Studiendesign & Heilung (\%) & Rezidive (\%) & $\begin{array}{l}\text { Kontinenz- } \\
\text { störung (\%) }\end{array}$ & $\begin{array}{l}\text { Follow-up } \\
\text { (Monate) }\end{array}$ \\
\hline $\begin{array}{l}\text { Champagne } \\
{[60]}\end{array}$ & 2006 & 46 & II, III & 0 & Prospektiv & 85 & $2,6(1 / 39)$ & k. A. & $12(6-24)$ \\
\hline Johnson [199] & 2006 & 15 & $\|$ & 0 & $\begin{array}{l}\text { Randomisiert } \\
\text { VG: } \bullet \text { Tab. } 14\end{array}$ & 60 & k. A. & k. A. & 3 \\
\hline Safar [355] & 2007 & 32 & II & $11(4 / 32)$ & Retrospektiv & 14 & k. A. & k. A. & 4 \\
\hline $\begin{array}{l}\text { Van Koperen } \\
\text { [450] }\end{array}$ & 2007 & 17 & I, II, Rezidiv & $6(1 / 17)$ & Prospektiv & 41 & k. A. & k. A. & $7(3-9)$ \\
\hline Ellis [117] & 2007 & 13 & II & 0 & Retrospektiv & 92 & k. A. & k. A. & $10(6-22)$ \\
\hline $\begin{array}{l}\text { Christoforidis } \\
\text { [74] }\end{array}$ & 2008 & 47 & I, II, III, RVF & $6(3 / 47)$ & Retrospektiv & 43 & k. A. & k. A. & $6,5(3-11)$ \\
\hline $\begin{array}{l}\text { Van Koperen } \\
{[448]}\end{array}$ & 2008 & 60 & II (distal) & 0 & $\begin{array}{l}\text { Randomisiert }^{\text {b }} \\
\text { VG: - Tab. } 9\end{array}$ & k. A. & k. A. & k. A. & k. A. \\
\hline Lawes [226] & 2008 & 20 & I, II, Rezidiv & k. A. & Retrospektiv & 24 & $\begin{array}{l}\text { k. A. (5-mal } \\
\text { Rezidivabszess) }\end{array}$ & k. A. & 7,4 \\
\hline $\begin{array}{l}\text { Schwandner, O. } \\
\text { [375] }\end{array}$ & 2008 & $\begin{array}{l}12 \\
7\end{array}$ & II & 0 & Prospektiv & $46(5 / 11)$ & k. A. & 0 & 9 \\
\hline Song $^{c}[403]$ & 2008 & 30 & I, II, III? & k. A. & Retrospektiv & 100 & k. A. & 0 & 0,5 \\
\hline $\begin{array}{l}\text { Echenique } \\
\text { [113] }\end{array}$ & 2008 & 23 & k. A. & k. A. & Retrospektiv & $60(14 / 23)$ & k. A. & k. A. & k. A. \\
\hline $\begin{array}{l}\text { Thekkinkattil } \\
\text { [425] }\end{array}$ & 2009 & 36 & II, III & $16(6 / 38)$ & Prospektiv & 50 & k. A. & k. A. & 6 \\
\hline Ortiz [298] & 2009 & 15 & II (distal) & 0 & $\begin{array}{l}\text { Randomisiert }{ }^{\mathrm{b}} \\
\text { VG: } 0 \text { Tab. } 9\end{array}$ & 20 & k. A. & k. A. & 12 \\
\hline Wang [472] & 2009 & 29 & II & 0 & $\begin{array}{l}\text { Retrospektiv } \\
\text { VG: T Tab. } 9\end{array}$ & 34 & k. A. & k. A. & 9 \\
\hline Chung [77] & 2009 & 27 & II & 0 & $\begin{array}{l}\text { Prospektiv } \\
\text { VG: } 0 \text { Tab. 5, } 9 \\
\text { und } 14\end{array}$ & 59 & k. A. & k. A. & 3 \\
\hline Adamina [11] & 2009 & 12 & II & 0 & $\begin{array}{l}\text { Prospektiv } \\
\text { VG: } \bullet \text { Tab. } 10\end{array}$ & 50 & k. A. & k. A. & k. A. \\
\hline Zubaidi [504] & 2009 & 22 & II (distal) & k. A. & Prospektiv & 83 & k. A. & k. A. & 12 \\
\hline $\begin{array}{l}\text { Christoforidis } \\
\text { [75] }\end{array}$ & 2009 & 37 & $\|$ & 0 & $\begin{array}{l}\text { Randomisiert } \\
\text { VG: } \bullet \text { Tab. } 10\end{array}$ & 32 & 42 & 0 & 6 \\
\hline $\begin{array}{l}\text { Schwandner, T. } \\
\text { [376] }\end{array}$ & 2009 & 60 & II & 0 & Prospektiv & 93 & 32 & k. A. & 12 \\
\hline Garg [138] & 2009 & 21 & II (distal) & 0 & Prospektiv & 71 & k. A. & k. A. & 10 \\
\hline Ellis [122] & 2010 & 63 & II, III, RVF & 19 & Retrospektiv & 81 & k. A. & 0 & 12 \\
\hline Lenisa [232] & 2010 & 63 & k. A. & $\mathrm{Ja}$ & Retrospektiv & 76 & k. A. & k. A. & $13(2-34)$ \\
\hline McGee [259] & 2010 & 41 & I-III & 0 & Prospektiv & $\begin{array}{l}43 \\
\text { Fistellänge: } \\
<4 \text { cm: } 21 \\
>4 \text { cm: } 61\end{array}$ & k. A. & 0 & $25(7-43)$ \\
\hline Owen [300] & 2010 & 32 & $\begin{array}{l}\text { I-III, } \\
\text { Pouch-anal }\end{array}$ & $9(3 / 32)$ & Retrospektiv & $\begin{array}{l}37 \\
\text { Kryptoglandulär: } \\
35\end{array}$ & k. A. & k. A. & $15(2-29)$ \\
\hline $\begin{array}{l}\text { Muhlmann } \\
\text { [276] }\end{array}$ & 2010 & $\begin{array}{l}22 \text { (Ge- } \\
\text { samt: } \\
55 \\
\text { Patien- } \\
\text { ten, } 70 \\
\text { Opera- } \\
\text { tionen) }\end{array}$ & II, III, RVF & 57 & $\begin{array}{l}\text { Retrospektiv } \\
\text { VG: } 0 \text { Tab. } 9\end{array}$ & $32(7 / 22)$ & k. A. & k. A. & $4(1-12)$ \\
\hline
\end{tabular}


Tab. 17 Ergebnisse von Fistelkürettage und Surgisis ${ }^{\circledR}$-Analfistelplug (Fortsetzung)

\begin{tabular}{|c|c|c|c|c|c|c|c|c|c|}
\hline Autor & Jahr & $\begin{array}{l}\text { Anzahl } \\
(n)\end{array}$ & Fisteltyp & $\begin{array}{l}\text { Anteil } \\
\text { M. Crohn } \\
(\%)\end{array}$ & Studiendesign & Heilung (\%) & Rezidive (\%) & $\begin{array}{l}\text { Kontinenz- } \\
\text { störung (\%) }\end{array}$ & $\begin{array}{l}\text { Follow-up } \\
\text { (Monate) }\end{array}$ \\
\hline $\begin{array}{l}\text { Lupinacchi } \\
\text { [246] }\end{array}$ & 2010 & 15 & II, RVF & 20 & Retrospektiv & $\begin{array}{l}3 \text { Monate: } 40 \\
7 \text { Monate: } 53 \\
\text { M. Crohn: } 33 \\
\text { (Plug-Verlust } \\
\text { 3/15) }\end{array}$ & k. A. & k. A. & $8 \pm 3$ \\
\hline Buchberg [55] & 2011 & 16 & k. A. & 0 & $\begin{array}{l}\text { Retrospektiv } \\
\text { VG: D Tab. } 18\end{array}$ & $13(2 / 16)$ & k. A. & k. A. & 3 \\
\hline $\begin{array}{l}\text { Van Koperen } \\
\text { [449] }\end{array}$ & 2011 & 30 & II, III & 0 & $\begin{array}{l}\text { Randomisiert, } \\
\text { multizentrisch } \\
\text { VG: - Tab. } 9\end{array}$ & 29 & k. A. & $\begin{array}{l}\text { Vaizey-Score } \\
\text { postop. leicht } \\
\text { erhöht } \\
\text { Wexner-Score } \\
\text { gleich }\end{array}$ & 11 \\
\hline Kleif [210] & 2011 & 37 & $0, I, I I, R V F$ & 3 & Retrospektiv & $\begin{array}{l}46 \\
\text { Plug-Verlust: } 30 \%\end{array}$ & k. A. & 0 & $4(1-41)$ \\
\hline Chan [62] & 2012 & 44 & II, III & $9(4 / 44)$ & Prospektiv & $\begin{array}{l}50 \text { (22/4) } \\
35 \text { (23/62 Plugs) }\end{array}$ & k. A. & 0 & $11(1,5-39)$ \\
\hline Cintron $^{d}[78]$ & 2013 & 73 & II, III & 11 & Prospektiv & $\begin{array}{l}38 \\
\text { Crohn: } 50(4 / 8)\end{array}$ & k. A. & k. A. & 15 \\
\hline Hall [165] & 2014 & 10 & I, II, RVF & 12 & $\begin{array}{l}\text { Prospektiv, } \\
\text { multizentrisch } \\
\text { (16 Operateu- } \\
\text { re) } \\
\text { VC: } \\
\text { - Tab. } 9,13 \\
\text { und } 17\end{array}$ & 20 & k. A. & $\begin{array}{l}\text { CCFI postop. } \\
\text { niedriger }\end{array}$ & 3 \\
\hline Blom [43] & 2014 & 126 & "Komplex" & 16 & $\begin{array}{l}\text { Retrospektiv, } \\
\text { multizentrisch }\end{array}$ & $24(30 / 126)$ & k. A. & k. A. & $13(1-47)$ \\
\hline Adamina [12] & 2014 & 46 & "Komplex" & 0 & Retrospektiv & k. A. & $\begin{array}{l}31 \text { (6 Monate) } \\
48 \text { (24 Monate) } \\
57 \text { (68 Monate) }\end{array}$ & $\begin{array}{l}0 \text { (eher Ver- } \\
\text { besserung!) }\end{array}$ & $12(0-82)$ \\
\hline Fisher [128] & 2014 & 31 & I-IV & 16 & $\begin{array}{l}\text { Retrospektiv } \\
\text { VG: - Tab. } 9\end{array}$ & 61 & k. A. & k. A. & $6(2-12)$ \\
\hline Köckerling ${ }^{\mathrm{e}}$ [213] & 2014 & $\begin{array}{l}52(\mathrm{NU}: \\
40)\end{array}$ & I, II, RVF & 5 & Retrospektiv & 90 & k. A. & 0 & $19 \pm 7$ \\
\hline \multicolumn{10}{|c|}{$\begin{array}{l}{ }^{2} \text { Randomierung vs. Fibrinkleber } \\
\text { b'Randomisierung vs. Mukosa-Flap } \\
\text { 'Randomisierung vs. Endorectal-Advancement-Flap, z. T. multi } \\
\text { kryptoglandulär) berücksichtigt } \\
{ }^{\text {cPPlug-Verlust } 9 \%, 4-m a l ~ p o s t o p e r a t i v e r ~ A b s z e s s ~} \\
{ }^{e} \text { Mit Exzision des extrasphinktären Fistelanteils, Button-Plug! }\end{array}$} \\
\hline
\end{tabular}

operativen Maßnahmen. In der Literatur wird dabei am häufigsten eine sekundäre Spaltung der Restfistel („two-stage fistulectomy“) beschrieben (-Tab. 6). Dabei handelt es sich um 12 Beobachtungsstudien, die überwiegend vor 1995 publiziert wurden und Heilungsraten von nahezu $100 \%$ angeben. Lediglich eine aktuellere Arbeit aus dem Jahr 2008 gibt ein differenzierteres Bild mit Heilungsraten von $60 \%$. Angaben zu sekundären Rezidiven fehlen in allen Publikationen. Verbunden ist dies jedoch mit einer hohen Rate an Kontinenzstörungen. Insgesamt schwan- ken diese Angaben in der Literatur zwischen 0 und $70 \%$.

Aus diesem Grunde kommt der fibrosierende Faden heute hauptsächlich bei trans- oder suprasphinktären Fisteln zur Anwendung. Die Einlage der Fadendrainage wird i. d. R. mit einer partiellen distalen Fistulektomie kombiniert.

Eine definitive Sanierung unter Schonung des Sphinkters durch eine sekundäre Fisteloperation wird nach Abheilung der akuten Entzündung angestrebt. Ziel dieses Verfahrens ist die Stabilisierung des Fistelgangs während des Abklin- gens der akuten Entzündung. Ein Vorteil für den Erfolg einer plastischen Fistelrekonstruktion scheint sich hierdurch jedoch nicht zu ergeben [273]. Andererseits spielt die Fadendrainage jedoch für die Plug-Anwendung eine wichtige Rolle, da der Durchzug des Plugs erleichtert wird [232].

Die drei neuen Publikationen fügen dem vorhandenen Wissen keine neuen Aspekte hinzu. 


\begin{tabular}{|c|c|c|c|c|c|c|c|c|c|}
\hline Autor & Jahr & $\begin{array}{l}\text { Anzahl } \\
\text { (n) }\end{array}$ & Fisteltyp & $\begin{array}{l}\text { Anteil } \\
\text { M. Crohn } \\
\text { (\%) }\end{array}$ & Studiendesign & Heilung (\%) & $\begin{array}{l}\text { Rezidive } \\
(\%)\end{array}$ & $\begin{array}{l}\text { Kontinenz- } \\
\text { störung (\%) }\end{array}$ & $\begin{array}{l}\text { Follow-up } \\
\text { (Monate) }\end{array}$ \\
\hline Buchberg [55] & 2011 & $\begin{array}{l}10 \\
\text { (11 Plugs) }\end{array}$ & k. A. & 0 & $\begin{array}{l}\text { Retrospektiv } \\
\text { VG: • Tab. } 17\end{array}$ & $55(6 / 11)$ & k. A. & k. A. & 2 \\
\hline De la Portilla [94] & 2012 & 19 & II & 0 & Retrospektiv & $16(3 / 19)$ & k. A. & $5(1 / 19)$ & 12 \\
\hline Ommer [294] & 2012 & 12 & II & 0 & Retrospektiv & $50(6 / 12)$ & k. A. & 0 & 6 \\
\hline Ommer [291] & 2012 & 40 & II, III & $4 / 40$ & Retrospektiv & $48(19 / 40)$ & k. A. & 0 & 6 \\
\hline Ratto [339] & 2012 & 11 & "Komplex" & 0 & Retrospektiv & $73(8 / 11)$ & k. A. & 0 & 5 \\
\hline Heydari [177] & 2013 & 48 & I, II & 0 & Retrospektiv & $69(34 / 48)$ & k. A. & 0 & $6(3-12)$ \\
\hline Stamos $^{\mathrm{a}}$ [405] & 2015 & 93 & I, II, III & 0 & Prospektiv & $\begin{array}{l}41(30 / 73) \\
49(36 / 74)\end{array}$ & k. A. & $\begin{array}{l}0 \\
\text { Wexner-Score } \\
\text { signifikant } \\
\text { besser }\end{array}$ & $\begin{array}{l}6 \\
12\end{array}$ \\
\hline
\end{tabular}

\section{Schneidender Faden („,cutting seton")}

Ziel dieses Verfahrens ist eine sukzessive Durchtrennung der vom Fistelgang umschlossenen Sphinkteranteile nach Ausräumung des entzündlichen Areals. Es kommen verschiedene Materialien als Faden zur Anwendung. Entweder handelt es sich um elastische Fäden, die sukzessive durchschneiden, oder es ist eine regelmäßige Nachspannung des Fadens erforderlich. Diese Methode wurde erstmals bereits von Hippokrates beschrieben.

Einen Sonderfall stellt der sog. chemische Faden aus Ayurvedamaterial dar. Für die Ayurvedaherstellung kommen 3 Grundstoffe zur Anwendung: Latex aus Euphorbia neriifolia; alkalische Puder aus der Asche von Achyranthes aspera und Puder von getrockneten Curcuma longa. Das Prinzip der Ayurvedafadendrainage besteht in der lockeren Einlage eines mit dem entsprechenden Medikament versehenen Fadens (Ksharasootra), der wöchentlich gewechselt wird. Ziel der Behandlung ist der spontane Fadenverlust nach chemischer Durchtrennung des durchbohrten Fistelgewebes. In mehreren indischen Studien [101, 102, 274] wurde eine hohe Abheilungsrate bei niedrigen Rezidiv- und Inkontinenzraten gesehen. Bemerkenswert ist die Arbeit von Mohite et al. [274]. Nach Einlegen eines Ayurvedafadens wird eine Heilung aller Fisteln ohne Kontinenzeinbußen bei 100 Patienten beobachtet. Andererseits beschreibt eine weitere Studie mit prospektiver Erfassung nach 3 Monaten lediglich eine Abheilungsrate von $33 \%$ [77]. Shukla et al. [390] vergleichen in einer großen randomisierten Studie die Ayurvedafadentherapie mit einer operativen Behandlung (265 vs. 237 Patienten) bei verschiedenen Fisteltypen und beobachten in beiden Gruppen eine 100 \%ige Heilungsrate. Nach 1 Jahr und bei einer Follow-up-Rate von lediglich $60 \%$ in beiden Gruppen betrug die Rezidivrate $4 \%$ in der Ayurvedagruppe und $11 \%$ in der Operationsgruppe (Unterschied statistisch signifikant). Die Inkontinenzrate betrug $5 \%$ in der ersten und $9 \%$ in der zweiten Gruppe. Diese Erfahrungen müssen jedoch unter heutigen wissenschaftlichen Anforderungen mit Vorsicht interpretiert werden.

Die Therapie mittels Ayurvedafaden wird auch in aktuellen chinesischen Studien [336] ausgewertet. Wang et al. [470] berichten über 60 Patienten mit inter- und transsphinktären Fisteln, die in 2 Gruppen randomisiert wurden (A: Exzision des Internus und der äußeren Fistelöffnung mit anschließender Fadeneinlage; B: Fistulotomie). Der Schmerzscore war in beiden Gruppen gleich, die Heilung betrug in Gruppe A $97 \%$ und in Gruppe B $100 \%$. Kontinenzveränderungen wurden nicht beobachtet.

Eine weitere randomisierte Studie [110], die den Ayurvedafaden mit der Fistulektomie vergleicht, ergab höhere Schmerzscores nach Fistelektomie sowie eine verlängerte Heilungsdauer bei eher kürzerer Arbeitsunfähigkeit nach Fadeneinlage. Die Rate an Kontinenzstörungen war nach Fadeneinlage niedriger als nach Fistulektomie (4 vs. $12 \%$ ).

Die Ergebnisse des schneidenden Fadens sind in 42 Studien aus den Jahren 1976 bis 2015 ausgewertet worden (ब Tab. 7). Die 6 neuen Publikationen fügen dem vorhandenen Wissen keine neuen Aspekte hinzu.

Fast alle Studien bieten ein gemischtes Krankengut mit Einschluss nahezu aller Fisteltypen. Die überwiegende Zahl der Studien bietet eine retrospektive Aufarbeitung des Patientenguts in Form von Beobachtungsstudien. Insgesamt liegt die Heilungsrate zwischen 80 und $100 \%$. Die übermittelten Raten an Kontinenzstörungen lagen zwischen 0 und $92 \%$. Mehrere Studien verweisen jedoch auf eine inakzeptabel hohe Inkontinenzrate $[46,166]$.

Eine weitere randomisierte Studie stammt aus dem Jahr 2001 [182]. Hier wird ein Vergleich zwischen einem schneidenden Faden aus Ayurvedamaterial und der primären Fistelexzision gezogen. Bei einer Heilungsrate von $100 \%$ in beiden Gruppen war die Rate der Kontinenzstörungen in der Ayurvedagruppe etwas höher. Eine weitere Studie von Zbar et al. [496] randomisiert die Anlage des "cutting seton“ in Fälle mit und ohne Schonung des M. sphincter ani internus. Bei ebenfalls kompletter primärer Heilungsrate war die Rate an Kontinenzstörungen ohne Schonung des M. sphincter ani internus etwas höher 


\begin{tabular}{|c|c|c|c|c|c|c|c|c|c|}
\hline Autor & Jahr & $\begin{array}{l}\text { Anzahl } \\
(n)\end{array}$ & Fisteltyp & $\begin{array}{l}\text { Anteil } \\
\text { M. Crohn } \\
(\%)\end{array}$ & Studiendesign & Heilung (\%) & Rezidive (\%) & $\begin{array}{l}\text { Kontinenz- } \\
\text { störung (\%) }\end{array}$ & $\begin{array}{l}\text { Follow-up } \\
\text { (Monate) }\end{array}$ \\
\hline A ba-bai-ke-re ${ }^{a, b}[1]$ & 2010 & 45 & $\mathrm{I}, \mathrm{II}$ & 0 & $\begin{array}{l}\text { Randomisiert } \\
\text { VG: } 0 \text { Tab. } 10\end{array}$ & 95 & k. A. & 2 & 5 \\
\hline $\operatorname{Han}^{\mathrm{a}}[171]$ & 2011 & 114 & ॥ & 0 & Retrospektiv & $52(62 / 114)$ & k. A. & 2 & $20(11-46)$ \\
\hline Öztürk [301] & 2015 & 10 & II, III & 10 & Retrospektiv & 90 & 20 & k. A. & 6 \\
\hline \multicolumn{10}{|c|}{$\begin{array}{l}\text { aHumaner azellulärer Hautmatrix-Plug } \\
\text { bPlug-Dislokation: } 11 \% \text {, Abszess: } 1 \% \\
\text { 'Plug aus autologem Ohrknorpel }\end{array}$} \\
\hline
\end{tabular}

bei gleichzeitig diskret niedrigerer $\mathrm{Re}$ zidivrate. Die Unterschiede sind nicht statistisch signifikant. Die randomisierte Studie von Altomare et al. [20] randomisiert den schneidenden Faden gegen die Fibrinkleberapplikation. Behandelt wird hier ein relativ homogenes Patientengut lediglich transsphinktärer Analfisteln kryptoglandulärer Genese. Kontinenzstörungen (gemessen mit dem WexnerInkontinenz-Score) traten in der Gruppe mit „cutting seton“ häufiger auf. Die Heilungsrate war jedoch in der Cuttingseton-Gruppe mit $88 \%$ signifikant höher als in der Fibringruppe mit $39 \%$.

Die aktuellste Arbeit stammt aus dem Jahr 2016 und umfasst 121 Patienten mit transsphinktären kryptoglandulären Fisteln, die alle vom selben Chirurgen operiert wurden [354]. Trotz eines Gesamtbeobachtungszeitraums von 14 Jahren (2000-2014) ist die Nachbeobachtungszeit mit $5 \pm 3$ Monaten sehrkurz. Die primäre Heilungsrate war mit 92,6 \% sehr hoch. Interessant aber auch fragwürdig ist eine generelle Kontinenzverbesserung von $11 \%$ Patienten mit Kontinenzstörung präoperativ und $19 \%$ postoperativ. Lediglich 7,5 \% hatten eine neu aufgetretene Inkontinenz. Es liegt der Verdacht nahe, dass - wie in anderen Publikationen - die präoperative Belastung der Patienten durch die Fistelsekretion als Inkontinenz gewertet wurde. Ansonsten ist eine Kontinenzverbesserung durch eine spaltende Operation schlichtweg unmöglich.

Die Ergebnisse der verschiedenen Fadentechniken wurden bisher in mehreren Reviews untersucht. Das älteste aus dem Jahr 1995 [256] beschreibt die verschiedenen Techniken, bietet jedoch keine Literaturübersicht. Es wird konstatiert, dass sich Fäden über Jahrtausende als effektive Fisteltherapie erwiesen haben. Die Richtlinien der koloproktologischen Vereinigungen von Irland und Großbritannien [483] erklären 2007 die Methode des schneidenden Fadens zur bevorzugten Therapie bei hohen Analfisteln. Eine umfangreiche Aufarbeitung der Literatur wurde 2009 von Ritchie et al. [345] vorgelegt. Die mittlere Inkontinenzrate wurde mit $12 \%$ ermittelt. Sie stieg mit der Höhe der inneren Fistelöffnung. Die Autoren kommen zu der Schlussfolgerung, dass die Inkontinenzrate nach Anwendung des schneidenden Fadens inakzeptabel hoch ist, so dass andere sphinkterschonende Verfahren empfohlen werden. $\mathrm{Zu}$ ähnlichen Ergebnissen unter Einschluss spanischsprachiger Literatur kommt ein weiteres Review von Vial et al. aus dem Jahr 2010 [466]. Bei hoher Heilungsrate steigt die Inkontinenzrate bis $25 \%$. In der Schlussfolgerung wird insbesondere eine Schonung des M. sphincter ani internus gefordert. Ein weiteres Review aus dem Jahr 2012 beschreibt die verschiedenen Techniken und Materialien, ohne Schlussfolgerungen zu ziehen [415].

Fazit für die Praxis. Die wichtigste Funktion der Fadendrainage liegt in der Vorbereitung einer späteren definitiven Sanierung bei im Rahmen einer Abszessdrainage nachgewiesener hoher Analfistel. Die in älteren Studien favorisierte Technik des schneidenden Fadens führt zwar $\mathrm{zu}$ einer hohen Heilungsrate, ist jedoch mit einem relevanten Risiko für postoperative Kontinenzstörungen behaftet, so dass sie nur in Ausnahmefällen zur Anwendung kommen sollte. Eine Empfehlung für dieses Verfahren, wie sie in anderen Richtlinien [483] vorgenommen wird, sollte unter Berücksichtigung der aktuellen Literatur nicht aufrechterhalten werden. Eine Veränderung der Leitlinienempfehlungen durch neue Studien gegenüber der Erstfassung ergibt sich nicht.

- Evidenzlevel: 2a

- Empfehlungsgrad: B

- Konsensusstärke: starker Konsens

\section{Plastischer Verschluss}

Ziel der verschiedenen Verfahren ist die Exzision der Fistel und des kryptoglandulären Infektionsherds mit direkter Naht der Muskulatur im Bereich des inneren Fistelostiums. Es kann zwischen folgenden Verfahren unterschieden werden:

- direkte Naht ohne Verschiebelappen,

- Mukosa-(Submukosa-)Verschiebe-

lappen (Mukosa-Flap),

- Rektumvollwand-Verschiebelappen

(Rectal-advancement-Flap),

- anodermaler Verschiebelappen

(Anoderm-Flap).

Einige Autoren kombinieren die Fistelexzision mit einer Sphinkterotomie des M. sphincter ani internus bis zur Höhe des inneren Fistelostiums. Die äußere perianale Wunde bleibt i. d. R. offen.

\section{Direkte Naht ohne Verschiebelap- pen}

Einige Studien verzichten auf die Deckung des inneren Fistelostiums nach direkter Naht des Schließmuskels ([10, 25, 219, 428, 430]; - Tab. 8). Hierbei handelt es sich ausnahmslos um Beobachtungsstudien. Die Heilungsraten der 4 Beobachtungsstudien aus 3 Kliniken liegen zwischen 56 und $100 \%$, die Rate an Kontinenzstörungen zwischen 0 und $10 \%$. Die neue Publikation fügt dem vorhan- 


\begin{tabular}{|c|c|c|c|c|c|c|c|c|c|}
\hline Autor & Jahr & $\begin{array}{l}\text { Anzahl } \\
(n)\end{array}$ & Fisteltyp & $\begin{array}{l}\text { Anteil } \\
\text { M. Crohn } \\
(\%)\end{array}$ & Studiendesign & Heilung (\%) & Rezidive (\%) & $\begin{array}{l}\text { Kontinenz- } \\
\text { störung (\%) }\end{array}$ & $\begin{array}{l}\text { Follow-up } \\
\text { (Monate) }\end{array}$ \\
\hline Wilhelm ${ }^{\mathrm{a}}$ [482] & 2011 & 11 & „Komplex" & 0 & Retrospektiv & $82(9 / 11)$ & k. A. & 1 (Grad 1) & $7(2-11)$ \\
\hline Giamundo [143] & 2013 & 35 & II & k. A. & Prospektiv & $71(25 / 35)$ & $5,8(2 / 35)$ & 0 & $20(3-36)$ \\
\hline Öztürk [302] & 2014 & 50 & I, II & k. A. & Retrospektiv & $82(41 / 50)$ & k. A. & k. A. & $12(2-18)$ \\
\hline Giamundo [142] & 2015 & 45 & „Komplex" & k. A. & Retrospektiv & $71(32 / 45)$ & k. A. & 0 & $30(6-46)$ \\
\hline
\end{tabular}

denen Wissen keine neuen Aspekte hin$\mathrm{zu}$.

\section{Mukosa/Submukosa-Flap}

Alternativ kann ein Schutz der Sphinkternaht durch Deckung mit einem Verschiebelappen erfolgen. Dieser Lappen wird entweder aus Mukosa, Submukosa und oberflächlichen Anteilen des Internus (Mukosa/Submukosa-Flap) oder aus der kompletten Rektumwand (Rectal-Advancement-Flap) gewonnen.

Die Behandlung von hohen Analfisteln mittels Fistelexzision und plastischer Deckung des inneren Ostiums mit einem Mukosa/Submukosa-Verschiebelappen wird in 43 Beobachtungsstudien dargestellt (•Tab. 9). Insgesamt 12 neue Studien wurden identifiziert. Mehrere Studien stammen aus den gleichen Kliniken und bilden wahrscheinlich das gleiche Patientengut mehrfach ab. In der Regel werden trans- und suprasphinktäre Fisteln behandelt. Einige Studien [89, 103, 215, 475, 503] schließen auch rektovaginale Fisteln mit ein, ohne dass dieses bei den Ergebnissen gesondert ausgewertet wird. Die Heilungsrate liegt zwischen 12 und $100 \%$. Rezidive werden nur von wenigen Autoren gesondert dargestellt. Im Jahr 2006 wurde eine Studie mit einem LangzeitFollow-up vorgestellt [445]. Nach einer primären Heilungsrate von $88 \%$ wurde nach 12 Monaten eine kumulative Rezidivrate von $22 \%$, nach 2 Jahren von $44 \%$ und nach 48 bzw. 72 Monaten von $63 \%$ gesehen, das heißt, 4 Jahre postoperativ waren lediglich $37 \%$ der initial behandelten Patienten fistelfrei. Einige Fistelrezidive manifestierten sich auch als Reabszedierung. Eingeschränkt wird die Aussage dieser Studien leider durch den relativ hohen Anteil von CrohnPatienten (29\%). Im Gegensatz dazu sahen Mitalas et al. [270] nach einer initialen Heilungsrate von $68 \%$ und einer Langzeit-Nachbeobachtung von 92 Monaten keine Rezidive. Die Rate der Kontinenzstörungen zeigte ebenfalls eine hohe Varianzbreite zwischen 0 und $40 \%$ im Vergleich der Studien.

In mehreren Studien werden randomisierte Vergleichsgruppen vorgestellt.

In der ältesten randomisierten Studie sahen Ellis et al. [120] keinen Vorteil für die zusätzliche Anwendung von Fibrinkleber.

Von der Hagen et al. [447] vergleichen die Ergebnisse von Mukosa-Flap und Fibrinkleber bei jeweils 15 Patienten und einem Follow-up von etwa 50 Monaten. Die Heilung war in der Flap-Gruppe doppelt so hoch (Mukosa-Flap: 80 \%, Fibrin: $40 \%)$. Kontinenzbeeinträchtigungen wurden in keiner Gruppe gesehen.

Eine randomisierte Studie aus Ägypten vergleicht die Ergebnisse von $\mathrm{Mu}$ kosa- und Vollwand-Flap [208]. Die Rezidivraten waren in der VollwandFlap-Gruppe deutlich niedriger (10\% vs. $40 \%)$. Gleichzeitig war aber auch die Rate an Kontinenzstörungen leicht erhöht gegenüber der Mukosa-FlapGruppe.

In einer randomisierten Studie vergleichen Madbouly et al. [247] das LIFTVerfahren mit dem Mukosa-Flap. Bei einer relativ kurzen Nachbeobachtungszeit von 12 Monaten waren die Erfolgsraten von LIFT (74 \%) und Mukosa-Flap (67 \%) nahezu gleich. Lediglich die Dauer der Wundheilung war in der Flap-Gruppe länger (32 vs. 22 Tage). Die Daten bestätigen die übrige Literatur und zeigen realistische Ergebnisse für beide Verfahren.

Van Koperen et al. (2011; [449]) verglichen in ihrer Studie den Mukosa-Flap mit der Plug-Implantation und fanden im 11 Monats-Follow-up bei beiden Verfahren mit Rezidivraten von $52 \%$ bzw. $72 \%$ enttäuschende Heilungsraten bei gleichen funktionellen Ergebnissen.

Eine weitere randomisierte Studie [1] vergleicht die Ergebnisse des MukosaFlaps mit dem azellulären Plug. Bei den transsphinktären Analfisteln wurde eine Heilung durch den Plug bei $92 \%$ erzielt (Kontinenzstörungen: $4 \%$, Analdeformierung: $0 \%$ ) im Gegensatz zu $64 \%$ bei Behandlung mit dem Mukosa-Flap (Kontinenzstörungen: $14 \%$, Analdeformierung: $11 \%)$. Als Schlussfolgerung zeigte sich eine signifikant bessere Heilungsrate durch den Plug, während die Unterschiede bei Kontinenz und Analdeformierung nicht signifikant waren. Unter Berücksichtigung der übrigen Studien erscheinen die hohen Erfolgsraten für den Plug eher fraglich. Da dieser Plug-Typ in Deutschland nicht erhältlich ist, ist der Wert der Studie für eine deutsche Leitlinie eingeschränkt.

\section{Rektumvollwand-Flap}

Die Abgrenzung zur plastischen Deckung mittels Rektumvollwandlappen ist nicht in allen Fällen möglich. Überschneidungen mit dem Mukosa/ Submukosa-Lappen auch innerhalb der einzelnen Patientenkollektive sind sehr wahrscheinlich. Die Abgrenzung zwischen den beiden Techniken erfolgte deshalb nach bestem Wissen, soweit dies den jeweiligen Publikationen zu entnehmen ist.

Ergebnisse nach Rektumvollwandplastik wurden demzufolge in 24 Studien untersucht (- Tab. 10). Davon konnten 7 gegenüber der ersten Version neu identifiziert werden. Einige weitere Studien konnten wegen des hohen Anteils an Crohn-Patienten nicht berücksichtigt werden. Im Gegensatz zum Muko- 


\begin{tabular}{|c|c|c|c|c|c|c|c|c|c|}
\hline Autor & Jahr & $\begin{array}{l}\text { Anzahl } \\
(n)\end{array}$ & Fisteltyp & $\begin{array}{l}\text { Anteil } \\
\text { M. Crohn } \\
(\%)\end{array}$ & Studiendesign & Heilung (\%) & Rezidive (\%) & $\begin{array}{l}\text { Kontinenz- } \\
\text { störung (\%) }\end{array}$ & $\begin{array}{l}\text { Follow-up } \\
\text { (Monate) }\end{array}$ \\
\hline Meinero [260] & 2011 & $136^{\mathrm{a}}$ & $\begin{array}{l}\text { II, III, IV, } \\
\text { Hufeisen- } \\
\text { fistel }\end{array}$ & k. A. & Retrospektiv & $87(72 / 98)$ & k.A: & k. A. & 6 \\
\hline Schwandner ${ }^{b}[374]$ & 2013 & 13 & II, III, RVF & 100 & Prospektiv & 82 & k. A. & 0 & 9 \\
\hline Chowbey [70] & 2013 & 416 & k. A. & k. A. & Prospektiv & k. A. & 26 & k. A. & 12 \\
\hline Meinero [262] & 2014 & 203 & $\begin{array}{l}\text { II, III, IV, } \\
\text { Hufeisen- } \\
\text { fistel }\end{array}$ & 0 & Retrospektiv & $\begin{array}{l}70 \\
\text { Mit Stapler: } 74 \\
\text { Mit Naht: } 58\end{array}$ & k. A. & 0 & $15(6-69)$ \\
\hline Kochhar [212] & 2014 & 82 & k. A. & 0 & Retrospektiv & 84 & k. A. & 0 & 6 \\
\hline Walega [467] & 2014 & 20 & ॥ & k. A. & Retrospektiv & 78 & 17 & 0 & 10 \\
\hline
\end{tabular}

sa/Submukosa-Flap stammen deutlich mehr Arbeiten aus der Zeit nach 2000. Die Ergebnisse entsprechen weitgehend denen des Mukosa/Submukosa-Flaps mit Heilungsraten zwischen 33 und $100 \%$ sowie Inkontinenzraten zwischen 0 und $71 \%$.

Neben den Beobachtungsstudien stellen 6 Arbeiten randomisierte Ergebnisse vor.

Die randomisierte Studie von Perez et al. [320] vergleicht den Vollwandlappen mit der Fistelspaltung mit primärer Sphinkterrekonstruktion und findet gleiche Ergebnisse in Bezug auf Heilung und Kontinenz.

Gustafson et al. [163] vergleichen in einer weiteren randomisierten Studie die Durchführung des Vollwandlappens mit und ohne lokale Antibiotikatherapie (Gentamycin) und können keinen relevanten Unterschied nachweisen.

Die beiden anderen Studien [75, 298] randomisieren gegenüber dem Analfistelplug. Ortiz et al. [298] sehen eine signifikant höhere Heilungsrate nach plastischem Verschluss (87 vs. $20 \%$ ). Zu gleichen Ergebnissen kommen auch Christoforidis et al. [75]. Diese verweisen jedoch auf die relevante Anzahl von Kontinenzstörungen nach plastischem Verfahren. Von 23 nachuntersuchten Patienten wiesen $17 \%$ eine Inkontinenz II. oder III. Grades auf.

Eine weitere randomisierte Studie aus Ägypten vergleicht die Ergebnisse von Mukosa- und Vollwand-Flap [208]. Die Heilungsraten waren in der VollwandFlap-Gruppe deutlich höher (85\% vs.
$30 \%)$. Gleichzeitig war aber auch die Rate an Kontinenzstörungen leicht erhöht.

\section{Anoderm-Flap}

Die dritte Möglichkeit der plastischen Deckung des inneren Fistelostiums nach Fistelexzision stellt der anodermale Verschiebelappen dar. Hier erfolgt die Deckung mit einer plastischen Verschiebung von Anodermgewebe, entweder als mobilisiertes Anodermläppchen, V-YPlastik oder der gestielte Anodermlappen. Ein Vorteil des Anoderm-Flaps besteht insbesondere bei Patienten mit engem Analkanal (z. B. durch narbige Veränderungen nach Voroperationen), der eine komplette Exploration und FlapBildung möglicherweise verhindert. Die Ergebnisse werden in 10 Beobachtungsstudien dargestellt (•Tab. 11). Neue Arbeiten konnten nicht identifiziert werden. Wie bei den anderen plastischen Techniken variieren die Heilungsraten zwischen 46 und 95\%, die Raten der Kontinenzstörungen zwischen 0 und $30 \%$. In einer Studie [189] wird sogar über eine Verbesserung der Kontinenz bei $70 \%$ der Operierten berichtet. Möglicherweise ist hier die fehlende Sekretion aus der äußeren Fistelöffnung bewertet worden. Es liegen zwei randomisierte Studien vor. Ho et al. [181] vergleichen den anodermalen Lappen mit einer konventionellen Technik, die aus Spaltung oder Fadendrainageneinlage besteht. Die Heilungsrate in den beiden kleinen Patientengruppen $(n=10)$ war gleich, wobei zur Kontinenz keine Stellung bezogen wird. Ellis et al. [120] randomisieren mit oder ohne simultane Fibrinanwendung und findet keinen Vorteil für die Fibrinapplikation.

Fazit für die Praxis. Der plastische Verschluss hoher Analfisteln durch FlapTechniken stellt ein etabliertes Verfahren dar. Die primäre Heilungsrate kann mit $60-80 \%$ beziffert werden, wobei die Erfolgsrate nach Vollwand-Flap höher $\mathrm{zu}$ sein scheint. Eine Beeinträchtigung der Kontinenz wird mit unterschiedlicher Häufigkeit beschrieben und muss Bestandteil der Aufklärung sein, auch wenn sie deutlich niedriger als nach Spaltung ist.

- Evidenzlevel: $1 \mathrm{~b}$

- Empfehlungsgrad: A

- Konsensusstärke: starker Konsens

\section{Fistelexzision mit direkter Sphinkterrekonstruktion}

Bei der Fistelexzision mit primärer Rekonstruktion des Schließmuskels wird nach der kompletten Exzision der Fistel und des dazugehörigen entzündlichen Gewebes eine primäre Readaption des durchtrennten Sphinkterapparats durchgeführt. Die identifizierten 11 Beobachtungsstudien beinhalten i. d. R. hohe komplexe Fisteln (•Tab. 12). Die Heilungsrate wird mit 54-97\% angegeben, Kontinenzstörungen werden mit einer Häufigkeit von 4-32 \% beobachtet. Perez et al. [320] vergleichen, wie bereits oben dargestellt, in einer randomisierten Studie die Analfistelspaltung und primäre Sphinkterrekonstruktion mit dem En- 


\begin{tabular}{|c|c|c|c|c|c|c|c|c|c|}
\hline Autor & Jahr & $\begin{array}{l}\text { Anzahl } \\
(n)\end{array}$ & Fisteltyp & $\begin{array}{l}\text { Anteil } \\
\text { M. Crohn } \\
(\%)\end{array}$ & Studiendesign & Heilung (\%) & Rezidive (\%) & $\begin{array}{l}\text { Kontinenz- } \\
\text { störung (\%) }\end{array}$ & $\begin{array}{l}\text { Follow-up } \\
\text { (Monate) }\end{array}$ \\
\hline Prosst [329] & 2012 & 1 & II & 0 & Fallstudie & 100 & k. A. & k. A. & 8 \\
\hline Prosst [330] & 2013 & 10 & II, III & 0 & Prospektiv & 90 & k. A. & 0 & 6 \\
\hline Mennigen [264] & 2015 & 10 & II, III, AVF & 60 & Retrospektiv & $\begin{array}{l}70 \\
\text { Kryptoglandulär: } \\
50(2 / 4) \\
\text { Crohn: } 85(5 / 6)\end{array}$ & k. A. & 0 & $8(5-17)$ \\
\hline Gautier [140] & 2015 & $\begin{array}{l}17 \text { (19 } \\
\text { Clips) }\end{array}$ & II, III, RVF & 35 & Retrospektiv & 12 & k. A. & k. A. & $4(2-7)$ \\
\hline
\end{tabular}

dorectal-Flap bei hohen trans- und suprasphinktären Analfisteln kryptoglandulärer Genese. Er beobachtet bei einer 100 \%igen Heilungsrate in beiden Gruppen eine Rate an Kontinenzstörungen zwischen 15 und $18 \%$ sowie eine Rezidivrate von etwa $7 \%$, und sieht daher keinen relevanten Unterschied zwischen den beiden Verfahren.

Gegenüber der ersten Leitlinienversion konnten 3 weitere Studien identifiziert werden ( $\bullet$ Tab. 12). Sie bestätigen eine mit über $90 \%$ sehr hohe Heilungsrate in Verbindung mit Kontinenzstörungen bei 6-12\% der Operierten.

Insbesondere bei hohen Fisteln ist die Nahtdehiszenz nach Spaltung und Rekonstruktion mit einer großen Gefahr der Inkontinenz verbunden. Eine Revision ist in diesen Fällen unumgänglich, $z$. T. kann auch die Anlage eines passageren Kolostomas erforderlich sein. Insgesamt ist die Datenlage bei dieser Technik noch relativ schmal. Insbesondere der Stellenwert der Rekonstruktion auch kleinerer Sphinkterdefekte ist derzeit unklar.

In einem Review aus dem Jahr 2015 werten Ratto et al. [338] 14 Studien von niedriger Qualität aus. Die generelle Erfolgsrate wird mit $93 \%$ und die Rate der Patienten mit einer Kontinenzverschlechterung mit $12 \%$ angegeben. Gleichzeitig stieg in den Studien die Lebensqualität der Patienten deutlich an. Als Schlussfolgerung sehen die Autoren eine hohe Erfolgsrate bei einem Inkontinenzrisiko, das niedriger als nach einfacher Spaltung ist. Sie fordern weitere Studien.
Fazit für die Praxis. Die plastische Rekonstruktion hoher Analfisteln durch Spaltung und Sphinkterrekonstruktion stellt mittlerweile ein etabliertes Verfahren dar. Die primäre Heilungsrate kann mit bis zu $90 \%$ beziffert werden. Eine Beeinträchtigung der Kontinenz wird mit unterschiedlicher Häufigkeit beschrieben und muss Bestandteil der Aufklärung sein, auch wenn sie deutlich niedriger als nach Spaltung ist. Die Bedeutung, insbesondere auch für die Behandlung oberflächlicher Fisteln sollte noch weiter evaluiert werden.

- Evidenzlevel: 1b

- Empfehlungsgrad: A

- Konsensusstärke: starker Konsens

\section{LIFT-Verfahren}

Von Rojanasakul et al. [352] wurde im Jahr 2007 die Ligatur des intersphinktären Raums als sog. LIFT-Verfahren („ligation of the intersphincteric fistula tract") vorgestellt. Bei 18 Patienten mit tiefen transsphinktären oder Hufeisenfisteln wird eine Rezidivrate von 5,6\% ohne relevante Kontinenzeinbußen beschrieben [350]. Zu ähnlichen Ergebnissen kommen Shanwani et al. [386] bei einer Heilungsrate von $82 \%$. Kontinenzeinbußen wurden nicht beobachtet. Das Prinzip der Operation besteht in der Freilegung des Fistelgangs im Bereich des Intersphinktärraums. Nach beidseitiger Ligatur wird der Fistelgang dann durchtrennt.

Ein ähnliches Vorgehen wurde bereits 1993 von Matos et al. [255] vorgestellt. Bei einem heterogenen Patientengut $(n=13)$ mit Rezidivfisteln wird die Möglichkeit der Schließmuskelscho- nung beschrieben. Heilungsraten werden leider nicht erwähnt. Unterschiede der Techniken werden in einem Leserbrief diskutiert [351].

In den letzten Jahren ist eine Vielzahl von Fallstudien mit Ergebnissen erschienen, die überwiegend Heilungsraten von 40-95\% ermitteln (• Tab. 13). Insofern stellt das Verfahren bei gleicher Erfolgsrate grundsätzlich eine Alternative zu den Flap-Verfahren da. Ein Vorteil besteht in der Wahl eines neuen Zugangswegs zur Fistel, insbesondere bei der Rezidivfistel.

In einer vergleichenden Studie zwischen LIFT und Mukosa-Flap bei hohen Analfisteln erzielten Tan et al. [420] durch das erste Verfahren eine Heilung bei $63 \%$ und durch den Flap bei $93 \%$ der operierten Patienten. Ein Vergleich in Bezug auf Kontinenzstörungen fand nicht statt. In einer weiteren vergleichenden Studie dieser beiden Techniken sahen Mushaya et al. [278] keinen entscheidenden Unterschied in Bezug auf Rezidivrate und Kontinenzstörungen.

In einer randomisierten Studie vergleichen Madbouly et al. [247] das LIFTVerfahren mit dem Mukosa-Flap. Bei einer relativ kurzen Nachbeobachtungszeit von 12 Monaten waren die Erfolgsraten von LIFT (74 \%) und Mukosa-Flap (67 \%) nahezu gleich. Lediglich die Dauer der Wundheilung war in der Flap-Gruppe höher (32 vs. 22 Tage). Die Daten bestätigen die übrige Literatur und zeigen realistische Ergebnisse für beide Verfahren.

Eine Weiterentwicklung stellt das von Ellis [118] beschriebene BioLIFTVerfahren da. Nach Dissektion des intersphinktären Raums über eine paraanale Inzision wird eine $4 \times 7 \mathrm{~cm}$ 
große Biomembran (Surgisis Biodesign ${ }^{\odot}$ ) eingebracht. Bei 31 Patienten wird eine primäre Heilungsrate von $94 \%$ beschrieben.

Trotz der kurzen Zeit, in der die relevanten Arbeiten erschienen, wurde das LIFT-Verfahren bereits in mehreren Reviews untersucht: Vergara-Fernandez (18 Studien) [465], Alasari (13 Studien) [18], Yassin (13 Studien) [487], Sirany (26Studien) [398] und Hong (24 Studien) [186]. Die aktuellste Arbeit von Sirany et al. [398] bewertet 26 Studien (davon eine randomisierte Studie), die eine Heilungsrate von $47-95 \%$ beschreiben. Erschwerend für die Bewertung werden 7 verschiedene Operationstechniken angeführt. Wie in allen Reviews kommen sie zu der Schlussfolgerung, dass das LIFT-Verfahren eine vielversprechende neue Technik mit akzeptabler Erfolgsrate und minimaler Beeinflussung der Kontinenz darstellt, die aktuelle Literatur aber noch keine abschließende Beurteilung zulässt.

Fazit für die Praxis. Das LIFT-Verfahren („ligation of the intersphincteric fistula tract") stellt eine neue Therapieoption bei hohen Analfisteln dar. Heilungs- und Kontinenzraten unterscheiden sich nicht signifikant von denen der Flap-Techniken.

- Evidenzlevel: $1 \mathrm{~b}$

- Empfehlungsgrad: A

- Konsensusstärke: starker Konsens

\section{Biomaterialien}

\section{Fibrinkleber}

Die Verwendung von Fibrinkleber zur Behandlung von Analfisteln wurde erstmals 1991 von Hjortrup et al. beschrieben [180]. Das Prinzip besteht in der Kürettage des Fistelgangs, ggf. nach Vorbehandlung mit einer Fadendrainage, und anschließender Auffüllung desselben mit Fibrinkleber. Anhand eines Modellversuchs am Schwein postulieren Buchanan et al. [54] die Notwendigkeit der Kürettage des Granulationsgewebes vor Verwendung des Fibrinklebers als Voraussetzung für eine Heilung. Einige Autoren berichten über mehrere Fibrinanwendungen beiprimärem Therapieversagen. Singer et al. [397] beobachteten bes- sere Ergebnisse nach zusätzlichem direktem Verschluss des inneren Fistelostiums durch Naht. Die Ergebnisse in der Literatur (39 Publikationen) zeigen eine hohe Varianz (• Tab. 14). Acht neue Arbeiten konnten identifiziert werden. Die Heilungsraten werden zwischen 0 und $100 \%$ beschrieben. Nur 8 Studien machen Aussagen zur Kontinenz und sehen keine Beeinträchtigung. Überwiegend handelt es sich dabei um persönliche Erfahrungsberichte bei einem inhomogenen Patientengut mit verschiedensten Fisteltypen $[6,16,380,503]$.

Derzeit liegen 5 randomisierte Studien vor. Lindsey et al. [238] vergleichen die Fibrinkleberapplikation mit der konventionellen Fistelbehandlung. Von den einfachen (intersphinktären oder distal transsphinktären) Analfisteln konnten durch die Fistelspaltung 7 von 7, also $100 \%$ ohne Beeinträchtigung der Kontinenz geheilt werden. Durch Fibrinkleberinstillation gelang dies lediglich bei 3 von 6 (50\%), wobei einmal eine zweite Fibrininjektion erforderlich war. Von den komplexen Fisteln (proximal transsphinktär, suprasphinktär) konnte bei 6 von 13 (46\%) eine primäre und nach erneuter Fibrinkleberapplikation bei weiteren 3 Patienten eine sekundäre Heilung erzielt werden.

Johnson et al. [199] vergleichen in einer randomisierten Studie die Plug-Technik mit der Fibrinkleberapplikation und sehen deutlich bessere Ergebnisse für die Plug-Anwendung.

Ellis et al. [120] vergleichen in einer randomisierten Studie Mukosa-Flap bzw. Anoderm-Flap mit und ohne Fibrinkleberinstillation. Schlechtere Werte fanden sich insbesondere für die Kombination Mukosa-Flap mit Fibrinkleber.

In einer weiteren randomisierten Studie verglichen Altomare et al. [20] die Fibrinkleberapplikation mit dem schneidenden Faden und beobachteten deutlich höhere Erfolgsraten nach der Fadenmethode unter Inkaufnahme einer höheren Rate an Kontinenzstörungen.

Von der Hagen et al. [447] vergleichen die Ergebnisse von Mukosa-Flap und Fibrinkleber bei jeweils 15 Patienten und einem Follow-up von ca. 50 Monaten. Die Heilung war in der Flap-Gruppe doppelt so hoch wie in der Fibrinkleber-Gruppe (Mukosa-Flap: 80 \%, Fibrin: $40 \%$ ). Kon- tinenzbeeinträchtigungen wurden in keiner Gruppe gesehen.

Garcia-Olmo et al. [136] verglichen die Fibrinkleberanwendung mit der gleichzeitige Zugabe von Fettstammzellen in einer randomisierten Studie bei verschiedenen Fisteltypen. In der FibrinGruppe heilten lediglich $16 \%$ der Fisteln, während in der Stammzell-FibrinGruppe $71 \%$ der Fisteln abheilten. In der letzten Gruppe wiesen jedoch $18 \%$ ein Rezidiv innerhalb eines Jahres auf.

Eine weitere Studie [176] vergleicht die Fibrininstillation und die Anwendung von Stammzellen. In der Gruppe mit Stammzellen allein zeigte sich nach einem Jahr eine Heilung bei $57 \%$, in der Kombination Stammzellen plus Fibrin bei $52 \%$ und mit Fibrin allein bei $37 \%$. Da die Stammzelltherapie derzeit nicht als Standardtherapie angesehen werden kann, ist die Aussagekraft dieser beiden Studien gering.

In einer nicht randomisierten aber vergleichenden Studie sehen van Koperen et al. [453] für die Applikation von Fibrinkleber bei der Durchführung eines Rektumwand-Verschiebelappens keine Vorteile.

Die vorliegenden Arbeiten wurden bisher in 3 älteren Review-Artikeln [168, 238, 417] analysiert, die derzeit keinen Stellenwert mehr haben.

Neben dem Fibrinkleber wurden von einigen Autoren auch andere Gewebekleber verwendet. So berichten Jain et al. [197] und Barillari et al. [32] über ihre Erfahrungen mit dem synthetischen Klebstoff Cyanoacrylat mit relativ guten Ergebnissen in kleinen Fallgruppen. De la Portilla et al. [92] schildern die Anwendung von BioGlue ${ }^{\circledR}$ (gereinigtes Rinderserumalbumin und Glutaraldehyd). In einem Leserbrief berichten jedoch Abbas et al. [3] über ihre persönlichen Erfahrungen. Sie beobachteten bei 6 Patienten in keinem Fall eine Heilung. Gleichzeitig kam es bei 3 Patienten zur Ausbildung einer Abszedierung, die eine operative Reintervention erforderlich machte. Ähnliche Erfahrungen werden in einem Leserbrief von Mitalas et al. [269] berichtet. Sie verweisen insbesondere auf die starke lokale Entzündungsreaktion. Nachdem de la Portilla et al. in einer Entgegnung [92] ihre Ergebnisse verteidig- 


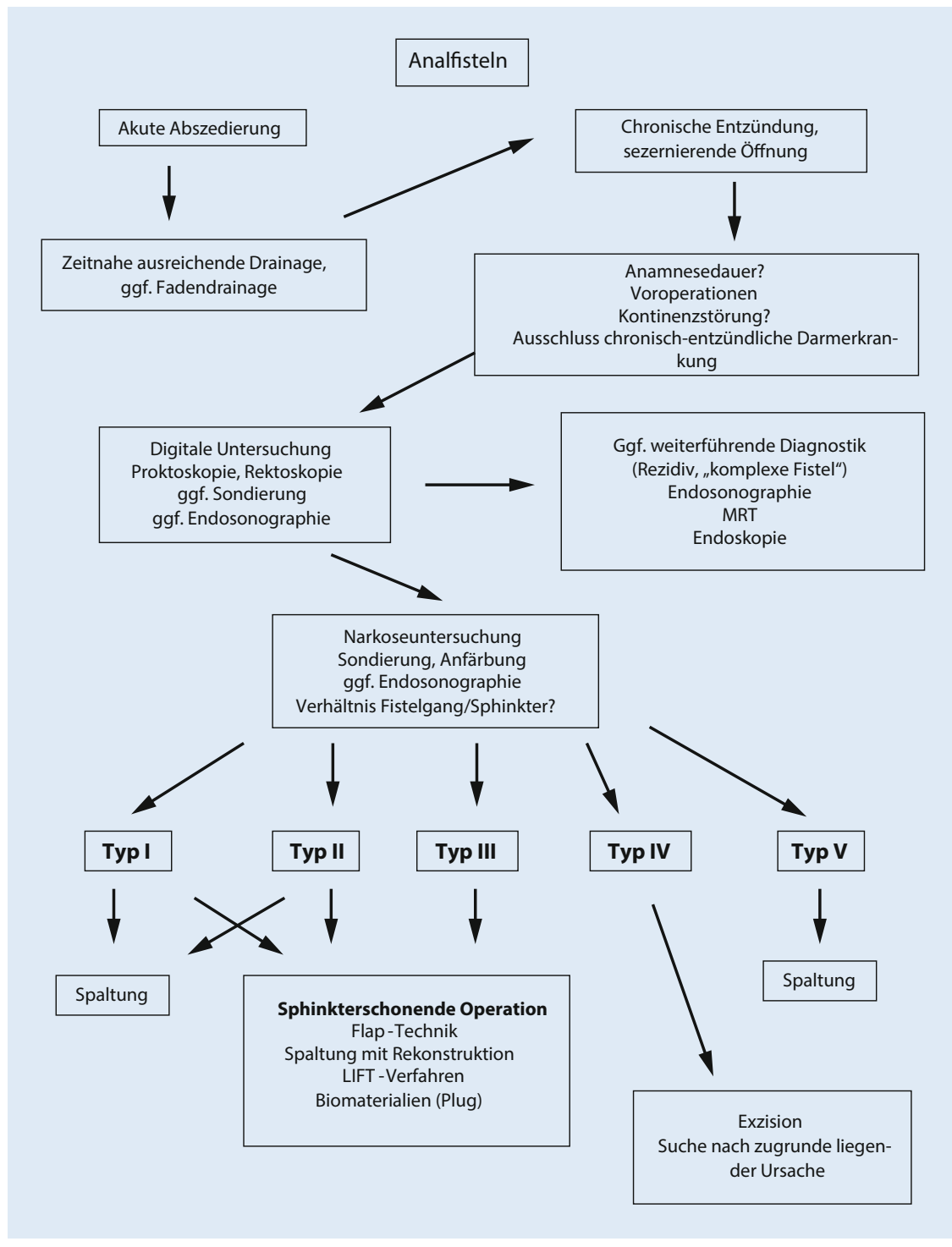

Abb. 3 A Diagnostik- und Therapiealgorithmus beim Analfistelleiden

ten, ergab das Langzeit Follow-up nach 60 Monaten, das in einer Kurzmitteilung veröffentlicht wurde [96], nur noch eine Langzeit-Heilungsrate von $21 \%$. Neuere Studien hierzu liegen nicht vor.

Fazit für die Praxis. Die primär guten Ergebnisse der Fibrinkleberanwendung bei hohen Analfisteln konnten im Langzeitverlauf sowie in neueren Publikationen nicht bestätigt werden, so dass die Anwendung speziellen Fällen vorbehalten sein sollte.

- Evidenzlevel: $1 \mathrm{~b}$

- Empfehlungsgrad: B

- Konsensusstärke: starker Konsens
Eine weitere Studie von Sileri et al. [395] beschreibt bei 11 Patienten eine Heilungsrate von $91 \%$ ohne Beeinträchtigung der Kontinenz.

Eine weitere Studie ist im Jahr 2016 erschienen [144]. Anhand einer Multizenterstudie aus 10 Kliniken mit $28 \mathrm{~Pa}$ tienten wird eine Erfolgsrate von $54 \%$ nach 12 Monaten beschrieben. Bei den intersphinktären Fisteln lag die Heilungsrate bei $67 \%$ und bei transsphinktären bei $44 \%$. Bei einem Patienten fand sich eine Verschlechterung der Kontinenz. Kritisch ist hier die niedrige Patientenzahl zu sehen (28 Patienten aus 10 Kliniken). Unklar ist auch die Fistelkanallänge von $0,5-5,5 \mathrm{~cm}$. Es bleibt unklar, wie ein 0,5-1 cm langer Kanal erfolgreich mit einer Substanz aufgefüllt werden soll.

Die Ergebnisse werden in - Tab. 15 dargestellt.

Fazit für die Praxis. Die derzeitige Studienlage zur Anwendung von Kollagen bei Analfisteln lässt noch keine endgültigen Schlussfolgerungen $\mathrm{zu}$.

- Evidenzlevel: 4

- Empfehlungsgrad: C

- Konsensusstärke: starker Konsens

\section{Injektion von autologen Stammzellen}

Die Injektion von autologen Stammzellen, teilweise in Verbindung mit weiteren Eingriffen, wurde erstmals 2009 beschrieben. In einer randomisierten Studie vergleichen Garcia-Olmo et al. [136] die Fibrinkleberanwendung mit der gleichzeitigen Zugabe von Fettstammzellen bei verschiedenen Fisteltypen. In der FibrinGruppe heilten lediglich $16 \%$ der Fisteln im Gegensatz zu $71 \%$ in der StammzellFibrin-Gruppe. In der letzten Gruppe wiesen jedoch $18 \%$ ein Rezidiv innerhalb eines Jahres auf. Ein Unterschied zwischen Crohn und Nicht-Crohn-Patienten wurde von den Autoren nicht beobachtet.

Eine weitere Studie [176] vergleicht die Fibrininstillation mit der Anwendung mit Stammzellen. In der Gruppe mit Stammzellen allein zeigte sich nach einem Jahr eine Heilung bei $57 \%$, in der Kombination Stammzellen plus Fibrin bei $52 \%$ und mit Fibrin allein bei $37 \%$. 
Da die Stammzelltherapie derzeit nicht als Standardtherapie angesehen werden kann, ist die Aussagekraft dieser beiden Studien gering.

In einer weiteren Pilotstudie wurde die Anwendung von autologen Stammzellen bei der operativen Behandlung von hohen transsphinktären Analfisteln mittels Mukosa-Flap untersucht. Bei einem medianen Follow-up von 26 Monaten heilten $90 \%$ der Fisteln. Kontinenzstörungen wurden nicht beobachtet [446].

Aktuell konnten insgesamt 7 Studien evaluiert werden ( $\bullet$ Tab. 16). Insgesamt finden sich Heilungsraten zwischen 35 und $90 \%$. Hinweise auf mögliche Kontinenzveränderungen bieten die Studien i. d. R. nicht.

Einen limitierenden Faktor für eine Anwendung in Deutschland dürften insbesondere die hohen Kosten darstellen.

Fazit für die Praxis. Die derzeitige Studienlage zur Anwendung von autologen Stammzellen bei Analfisteln lässt derzeit trotz Vorliegen randomisierter Studien noch keine endgültigen Schlussfolgerungen zu.

- Evidenzlevel: $1 \mathrm{~b}$

- Empfehlungsgrad: A

- Konsensusstärke: starker Konsens

\section{Analfistelplug}

Zunächst die Anwendung von Fibrinkleber und dann die kommerzielle Verbreitung des Analfistelplugs hat z. T. mit Unterstützung der Industrie zu einer neuen Beschäftigung mit dem Thema Analfistel geführt. So sind seit der ersten Publikation im Jahr 2006 bis heute 24 Arbeiten zum Thema Fistelplug erschienen. Im Gegensatz zu den sog. konventionellen Verfahren erfolgt keine Exzision des entzündlichen Gewebes, sondern lediglich eine Okklusion mit Fremdmaterial, so dass von einem gänzlich neuen Ansatz gesprochen werden muss, wie in mehreren Editorials koloproktologischer Publikationsorgane angesprochen wurde [242, 309].

Bei dem am häufigsten verwendeten und untersuchten Analfistelplug handelt es sich um ein biomedizinisches Produkt aus Dünndarmsubmukosa des Schweins, das unter dem Handelsnamen Surgisis ${ }^{\circledR}$ $\mathrm{AFP}^{\mathrm{T}}$ Anal Fistula Plug vertrieben wird.
Das Prinzip besteht in der Okklusion des Fistelkanals durch den konusförmigen Plug als Matrix für das Einsprossen von körpereigenem Gewebe. Die Sachkosten sind hoch.

Voraussetzung für die Verwendung des Plugs ist die Möglichkeit der Sondierung der Fistel, ggf. durch die vorherige Einlage einer Fadendrainage. Bevorzugte Indikation ist die hohe Analfistel. Einige Autoren kombinieren die PlugTechnik mit dem Verschluss des inneren Fistelostiums durch einen Verschiebelappen. Grundlagen und Indikationen des Analfistelplugs wurden 2007 in einem Konsensuspapier publiziert [86]. Die veröffentlichten 35 Literaturergebnisse bilden überwiegend Beobachtungsstudien ab (•Tab. 17). Insgesamt 11 neue Publikationen konnten identifiziert werden. Die Heilungsraten schwanken zwischen 14 und $93 \%$. Kontinenzstörungen werden i. d. R. nicht untersucht. Lediglich 3 Studien berichten über eine unveränderte Kontinenzleistung.

Vier randomisierte Studien liegen derzeit vor. Johnson et al. [199] vergleichen in einer randomisierten Studie die PlugTechnik mit der Fibrinkleberapplikation und sehen deutlich bessere Ergebnisse für die Plug-Anwendung. Kritisch ist hier anzumerken, dass die als Vergleich herangezogene Fibrintechnik kein etabliertes Verfahren darstellt und die anfangs publizierten guten Ergebnisse in größeren Studien mit längerem Follow-up nicht reproduziert werden konnten.

Ein Vergleich der Plug-Insertion mit der Flap-Technik wurde in mehreren Studien vorgestellt, wobei van Koperen et al. [448] zunächst lediglich das Studienprotokoll ohne Ergebnisse bieten. Ortiz et al. [298] sahen nach Fistelplug eine inakzeptabel hohe Rate an Rezidiven, was zu einer vorzeitigen Beendigung der Studie führte. Christoforidis et al. [75] beobachteten ebenfalls eine deutlich geringere Heilungsrate nach PlugAnwendung, andererseits zeigten nach der Flap-Technik deutlich mehr Patienten eine Kontinenzstörung. Van Koperen et al. [449] verglichen in ihrer Studie den Mukosa-Flap mit der Plug-Implantation und fanden im 11-Monats-Follow-up bei beiden Verfahren mit Rezidivraten von $52 \%$ bzw. $72 \%$ enttäuschende Hei- lungsraten bei gleichen funktionellen Ergebnissen. In einer nicht randomisierten vergleichenden Studie beobachteten Chung et al. [77] gleiche Ergebnisse nach Plug bzw. Advancement-Flap bei hohen Fisteln.

Eine Arbeit [259] untersucht die Bedeutung der Länge des Fistelgangs für die Erfolgsrate. Akzeptable Heilungsraten wurden erst ab einer Länge von $4 \mathrm{~cm}$ beobachtet. Unter $4 \mathrm{~cm}$ betrug die Heilung lediglich $21 \%$ und über $4 \mathrm{~cm} 61 \%$, so dass bei diesen Fisteln der Plug möglicherweise als erste Option angesehen werden kann [73].

Die neueren Publikationen liefern keine nennenswerten neuen Informationen, bestätigen aber die niedrige Heilungsrate, die deutlich unter $40 \%$ liegt.

Mit einem neuen Plug-Design (Button-Plug) konnte eine aktuelle Studie über eine Erfolgsrate von $90 \%$ berichten [213].

Festzuhalten ist die geringe Morbidität des Verfahrens. Auch wenn die übermittelten Daten über postoperative Kontinenzstörungen sehr selten sind, ist nicht von einer nennenswerten Beeinflussung der Kontinenz durch das Plug-Verfahren auszugehen. Ein derzeit noch ungelöstes Problem stellt der frühzeitige Plug-Verlust dar, der in den meisten Fällen mit einer Fistelpersistenz assoziiert ist.

Tierexperimentelle Arbeiten deuten darauf hin, dass eine Kürettage des Fistelgangs die Erfolgsrate steigen lässt [40].

In der Zwischenzeit wurde durch die Industrie ein weiterer Plug (Gore Bio-A Fistula Plug ${ }^{\circledR}$; Tab. 18) vorgestellt. Dieser besteht zu $67 \%$ aus Polyglykolsäure und $\mathrm{zu} 33 \%$ aus Trimethylencarbonat. Beide Stoffe werden über den Citratzylus in Kohlendioxid und Wasser abgebaut. Es handelt sich somit um ein langsam resorbierbares Biomaterial. Ein möglicher Vorteil gegenüber dem herkömmlichen Plug besteht in der besseren Fixationsmöglichkeit durch den Kopf und das größere Volumen des Plugs. Aktuell liegen 7 Studien vor. Sie beobachten Heilungsraten zwischen 16 und $73 \%$, so dass ein nennenswerter Vorteil gegenüber dem Surgisis-Plug bis jetzt nicht belegt werden konnte. Lediglich eine vergleichende Studie mit geringer Fallzahl beschreibt 
einen Vorteil für den Gore-Plug [55]. Alle Studien beschreiben eine geringe Beeinflussung der Kontinenzleistung.

Die in - Tab. 18 aufgeführten Publikationen wurden im Review von Narang et al. [281] aufgearbeitet. Die Evidenz wird auch hier als unzureichend bewertet. Es scheint jedoch eine sichere und einfache Methode darzustellen, die niedrige Komplikationsraten und eine geringe Beeinträchtigung der Kontinenz nach sich zieht.

\section{Neuere Plug-Materialien}

In weiteren Studien wurden andere Materialien (azelluläre Hautmatrix, Ohrknorpel) vorgestellt. Diese sind jedoch in Deutschland nicht verfügbar (• Tab. 19). Eine randomisierte Studie [1] vergleicht die Ergebnisse des Mukosa-Flaps mit dem azellulären Plug. Bei den transsphinktären Analfisteln wurde eine Heilung durch den Plug bei $92 \%$ erzielt (Kontinenzstörungen: 4\%, Analdeformierung: 0\%) im Gegensatz zu 64\% beim Mukosa-Flap (Kontinenzstörungen: $14 \%$, Analdeformierung: $11 \%$ ). Als Schlussfolgerung zeigte sich eine signifikant bessere Heilungsrate durch den Plug, während die Unterschiede bei Kontinenz und Analdeformierung nicht signifikant waren. Unter Berücksichtigung der übrigen Studien erscheinen die hohen Erfolgsraten für den Plug eher fraglich. Da dieser Plug-Typ in Deutschland nicht erhältlich ist, ist der Wert der Studie für eine deutsche Leitlinie eingeschränkt.

\section{Reviews zur Plug-Technik}

Insgesamt wurden die Plug-Technik und weitere neue Verfahren in diversen Reviews aufgearbeitet [82, 139, 214, 234, 292, 296, 332, 402]. Cirocchi et al. [82] sehen in ihrem Review keinen relevanten Vorteil der Biomaterialien gegenüber den herkömmlichen Verfahren und fordern weitere randomisierte Studien. O'Riordan et al. [296] sichteten 76 Arbeiten (auch Abstracts), von denen 20 ausgewertet wurden. Insgesamt wurden lediglich 530 Patienten ausgewertet (4-60 Patienten pro Studie). Ein Plugverlust wurde in $9 \%$ der Fälle beschrieben. Die Heilung betrug bei nicht Crohn-assoziierten Fisteln $54 \%$ und bei
Crohn-Fisteln $55 \%$. Im Review von $\mathrm{Pu}$ et al. [332], das die Plug-Methode mit der konventionellen Technik vergleicht (i. d. R. Spaltung), wird eine lediglich moderate Erfolgsrate mit hohen Rezidivraten beschrieben. Letztlich wird ein schonendes Verfahren konstatiert, wobei die Vorteile für die Kontinenz unklar bleiben. Ein weiteres Review aus dem Jahr 2015 von Köckerling et al. [214], das auch den Gore Bio-A Fistula-Plug ${ }^{\circledR}$ mit einbezieht, sieht bei komplexen Fisteln eine Erfolgsrate von $50-60 \%$ bei niedriger Komplikationsrate, fordert jedoch weitere und qualitativ bessere Studien.

Fazit für die Praxis. Die Einführung des Fistelplug-Verfahrens hat die Behandlung der hohen Analfisteln grundsätzlich durch neue Therapieoptionen erweitert. In randomisierten Studien wurde jedoch gegenüber den plastischen Verfahren eine deutlich niedrigere Heilungsrate von 20 bzw. $28 \%$ beschrieben. Auch weitere Materialien konnten die Erfolgsrate nicht relevant verbessern.

- Evidenzlevel: $1 \mathrm{~b}$

- Empfehlungsgrad: B

- Konsensusstärke: starker Konsensus

\section{Neue technische Verfahren}

\section{Laseranwendung}

Als neue Methode wurde die Fistelkoagulation durch eine Lasersonde ( $\mathrm{FiLaC}^{\circledR}{ }_{-}$ Biolitec), teilweise kombiniert mit einer Flap-Technik, vorgestellt (• Tab. 20). Die derzeit vorliegenden 4 Studien zeigen Erfolgsraten von $71-82 \%$ ohne nennenswerte Beeinflussung der Kontinenz. Weitere Aussagen sind aufgrund der Datenlage derzeit nicht möglich. Limitierende Faktoren für die Anwendung sind insbesondere hohe Anschaffungs- und Materialkosten.

\section{VAAFT-Methode}

Ein weiteres neues Verfahren stellt die videoassistierte Fistelbehandlung (VAAFT: „video-assisted anal fistula treatment") nach Meinero dar [260, 261, 493]. Dabei wird der Fistelgang videoendoskopisch assistiert sondiert, gespült, kürettiert, die Wand koaguliert und mit Fibrinkleber aufgefüllt. Das innere Fistelostium wird mit einem Klammer- nahtgerät (Contour ${ }^{\mathrm{TM}}$, Ethicon Endosurgery) oder durch direkte Naht verschlossen. Die Kosten für das Spezialinstrumentarium und das Klammernahtgerät sind hoch. Der Erstbeschreiber beobachtet Heilungsraten zwischen 58 und $87 \%$. Die Ergebnisse sind in - Tab. 21 dargestellt.

Während eine weitere Publikation [374] lediglich Crohn-Fisteln behandelt, konnte eine weitere Arbeit identifiziert werden, die eine Erfolgsrate von $84 \%$ bestätigt [212]. Eine polnische Studie aus dem Jahr 2014 [467] mit 20 Patienten und einem Follow-up von 10 Monaten konnte eine Heilungsrate von $63 \%$ erzielen. Kontinenzstörungen wurden nicht beobachtet.

Eine etwas variierte Technik wurde 2013 von Chowbey et al. [70] aus Indien publiziert. Insgesamt $94 \%$ der Eingriffe wurden tageschirurgisch durchgeführt! Nach einer mittleren Nachbeobachtungszeit von 12 Monaten wurden bei $26,1 \%$ der Operierten Rezidive festgestellt, wobei festzuhalten ist, dass lediglich 134 von 416 Patienten nachuntersucht wurden.

\section{OTSC-Clip}

Die OTSC-Clip-Technik wird endoskopisch im Magen-Darm-Trakt zum Verschluss von Wanddefekten nach Perforation oder Resektion eingesetzt. 2011 wurde erstmals die Anwendung bei Analfisteln mit einem spezifischen Setting beschrieben [331]. Derzeit vorliegende Studien (• Tab. 22) zeigen ein äußerst differentes Bild von Heilungsraten zwischen 12 und $90 \%$, so dass keine abschließende Bewertung möglich ist. Die Materialkosten sind relativ hoch. Bezüglich der Studie von Gautier et al. [140], die bei der Durchführung durch Gastroenterologen sehr schlechte Ergebnisse und vermehrte Komplikationen beschreibt, ist ein Kommentar erschienen, der die Schwächen dieser Studie diskutiert [371].

Fazit für die Praxis. Auch weitere technische Verfahren konnten gegenüber den herkömmlichen Verfahren derzeit noch keine Überlegenheit nachweisen. Die Studienlage ist noch unzureichend.

- Evidenzlevel: 4

- Empfehlungsgrad: 0 
Tab. 23 Gesamtdarstellung der Literatur anhand der vorhergehenden Tabellen

\begin{tabular}{|c|c|c|c|c|c|}
\hline Verfahren & Anzahl Studien & $\begin{array}{l}\text { Anzahl Patien- } \\
\text { ten }\end{array}$ & $\begin{array}{l}\text { Heilung Median } \\
(\%)\end{array}$ & $\begin{array}{l}\text { Rezidiv Median } \\
\text { (\%) }\end{array}$ & $\begin{array}{l}\text { Inkontinenz Median } \\
\text { (\%) }\end{array}$ \\
\hline Spaltung & 34 (1966-2015) & 4278 & 96 & 2 & $26(0-82)$ \\
\hline Loser Faden & 17 (1976-2014) & 1393 & 80 & k. A. & $26(0-63)$ \\
\hline Two-stage-Fistulektomie & 12 (1976-2014) & 928 & 96 & k. A. & $6(0-98)$ \\
\hline "Cutting seton" & $43(1966-2015)$ & 2369 & 100 & k. A. & $14(0-92)$ \\
\hline Direkte Naht & $5(1993-2012)$ & 419 & 72 & 6 & $13(0-55)$ \\
\hline Mukosa-Flap & $43(1983-2015)$ & 2461 & 72 & 6 & $13(0-43)$ \\
\hline Rektumwand-Flap & 24 (1988-2015) & 999 & 82 & 10 & $12(0-71)$ \\
\hline Anoderm-Flap & 10 (1996-2007) & 262 & 78 & 19 & $0(0-30)$ \\
\hline Spaltung/Rekonstruktion & $11(1985-2014)$ & 583 & 94 & 6 & $12(4-21)$ \\
\hline LIFT & $30(1993-2015)$ & 974 & 77 & 10 & $0(0-31)$ \\
\hline Fibrinkleber & 39 (1991-2011) & 1182 & 56 & 8 & k. A. \\
\hline Kollageninjektion & $3(2010-2016)$ & 68 & 71 & k. A. & k. A. \\
\hline Autologe Stammzellen & 7 (2009-2014) & 268 & 39 & k. A. & k. A. \\
\hline Fistelplug (Surgisis ${ }^{\circledR}$ ) & 35 (2006-2014) & 1240 & 48 & 37 & k. A. \\
\hline Fistel-Plug (Gore ${ }^{\circledR}$ ) & 7 (2011-2015) & 233 & 49 & k. A. & 0 \\
\hline Laseranwendung & $4(2011-2015)$ & 223 & 77 & k. A. & 0 \\
\hline VAAFT & $5(2011-2014)$ & 870 & 84 & k. A. & 0 \\
\hline OTSC-Klemme & $3(2012-2015)$ & 38 & 50 & k. A. & k. A. \\
\hline
\end{tabular}

- Konsensusstärke: starker Konsensus

\section{Sonstige Verfahren}

Verschiedene andere Verfahren werden in der Literatur in Form von Fallvorstellungen beschrieben. Im folgenden Absatz werden alle Verfahren beschrieben, die lediglich einmal oder nur durch eine weitere Publikation beschrieben wurden.

Von 2 Autoren wird die Exzision von Analfisteln mittels Radiofrequenzablation beschrieben. Gupta et al. [159] beobachteten bei 136 Patienten mit einem Follow-up von 18 Monaten und einer Anwendung bei oberflächlichen Fisteln eine Heilungsrate von 96,3\%. In einer randomisierten Studie und im Vergleich zur Fistelexzision mittels Diathermie sehen Filingeri et al. [127] einen Vorteil in Bezug auf die operative Anwendung und geringere Schmerzen bei gleichen Heilungsraten.

Tasci et al. [423] beschreiben die Fistelexzision mit einem eigens entwickelten Fistulotom, das mittels Führungsdraht die Fistel mit einem entsprechenden Rand ausschneidet. Die übermittelte Heilungsrate beträgt 12 von 13 Patienten bei einem Follow-up von 13 Monaten.
Perez et al. [318] berichten über $5 \mathrm{~Pa}$ tienten, bei denen hohe Fisteln in Analogie zur Stapler-Hämorrhoidopexie mit dem PPH-01-Stapler (Ethicon Endosurgery) erfolgreich behandelt wurden. In einem 26-monatigen Follow-up wurden weder relevante permanente Kontinenzstörungen noch Rezidive gesehen. Diese Methode dürfte jedoch speziellen Indikationen vorbehalten bleiben, da diese 5 Fisteln, die am ehesten als extrasphinktäre zu bezeichnen wären, in einem Beobachtungszeitraum von 1 Jahr nur extrem selten in einer Institution beobachtet werden dürften. Andererseits stellen extrasphinktäre Fisteln die einzige mögliche Indikation einer StaplerAnwendung dar, da alle anderen kryptoglandulären Fisteln definitionsgemäß auf Höhe der Linea dentata münden und die Erfahrungen der Stapler-Hämorrhoidopexie zeigen, dass die Klammerpositionierung in dieser Höhe zu deutlich vermehrten Schmerzen führt. Grundsätzlich sind diese Fisteln auch nicht Thema dieser Leitlinie.

Zhu et al. [498] stellten 2014 ein selbst entwickeltes Instrumentarium zur Fistelexzision vor. Nach einer Beobachtungszeit von 36 Monaten konnten 13 von
14 behandelten Patienten ohne Beeinflussung der Kontinenz geheilt werden.

Banasiewicz et al. [31] stellten 2015 eine Falldarstellung über den erfolgreichen Abschluss einer Therapie mit einem $\mathrm{VAC}^{\circledR}$-Verband bei einer Patientin mit rezidivierender transsphinktärer Fistel vor.

Fazit für die Praxis. Alternative Verfahren wurden bis jetzt lediglich in Fallstudien publiziert.

- Evidenzlevel: 5

- Empfehlungsgrad: 0

- Konsensusstärke: Konsensus

\section{Operative Therapie: Reviews}

Die Daten aller Studien sind in • Tab. 23 aufgearbeitet worden.

Die Literatur zur Behandlung der Analfisteln wurde in zahlreichen Reviews aufgearbeitet. $\mathrm{Zu}$ den Reviews vor 2010 [185, 248, 346, 384, 401] wird hier auf die erste Version der Leitlinien verwiesen [290]. In den vergangenen 5 Jahren wurden zahlreiche weitere Reviews publiziert. Da hier lediglich das bekannte Wissen ausgehend von meist minderwertigen Studien zusammengefasst wird, seien diese hier nur kursorisch 
genannt $[34,100,108,123,160,334,373$, 387, 407, 408].

Im Review von Limura et al. [236] werden konventionelle und neue Techniken vorgestellt. Aufgrund der niedrigen Evidenz und sehr unterschiedlicher Fisteltypen wird ein individuelles Vorgehen empfohlen.

Göttgens et al. [151, 152] identifizieren den Mukosa-Flap als das am besten untersuchte Verfahren. Trotz 14 vorliegender randomisierter Studien konnte kein bestes operatives Verfahren identifiziert werden.

Cadeddu et al. [57] sehen die konventionellen Verfahren als Standardverfahren und werten die neuen Techniken (Fibrin, Kollagen, Plugs, Stammzellen) als alternative Optionen, da Studien mit Langzeitergebnisse fehlen.

Eine Cochrane-Analyse wurde erstmals 2010 publiziert [195]. Sie kommt erwartungsgemäß zu ähnlichen Ergebnissen wie das Review von Malik. Es wurden 10 randomisierte Studien identifiziert, dieverschiedene Therapieverfahren vergleichen. Diese Studien werden in den vorliegenden Leitlinien in den entsprechenden Kapiteln ausgewertet. Bezüglich der Rezidivrate wurden keine relevanten Unterschiede zwischen den Studien gefunden, während die Inkontinenzrate nach Fibrinkleberanwendung durch die fehlende Sphinkterdurchtrennung niedriger war. Die Plug-Technik wird als noch nicht ausreichend evaluiertes Verfahren behandelt, obwohl gerade hier randomisierte Studien vorliegen, welche die Überlegenheit der plastischen Verfahren in Bezug auf die Heilungsrate bei gleichzeitig höherer Inkontinenzrate beschreiben. Abschließend wird die schlechte Qualität der vorliegenden Studien beklagt, und es werden bessere Studien gefordert. Eine neue Analyse liegt nicht vor.

In eine ähnliche Richtung zielen auch die publizierten Leitlinien anderer Fachgesellschaften, wobei hier auch die jahrelangen, jedoch nicht publizierten Erfahrungen koloproktologischer Zentren einfließen.

Im Jahr 2011 wurde von Steele et al. eine Revision der Leitlinien der Amerikanischen Gesellschaft für Koloproktologie vorgestellt [406, 480].
Folgende Aussagen zu den Analfisteln sind enthalten:

\section{Diagnostik.}

- Eine krankheitsspezifische Anamneseerhebung und klinische Untersuchung sollten erfolgen. (Starke Empfehlung, Evidenzlevel 1c).

- Untersuchungsmethoden wie Fistulographie, Endosonographie, Computertomographie und MRT sollten bei ausgewählten Patienten zur Diagnostik eingesetzt werden. (Starke Empfehlung, Evidenzlevel 1c).

\section{Einfache Analfisteln.}

- Einfache Analfisteln können gespalten werden. Durch eine Marsupialisation kann die Wundheilungsrate verbessert werden. (Starke Empfehlung, Evidenzlevel 1b).

- Einfache Fisteln können durch Kürettage und Fibrininjektion behandelt werden. (Schwache Empfehlung, Evidenzlevel 2c).

\section{Komplexe Analfisteln.}

- Komplexe Fisteln können durch Kürettage und Fibrininjektion behandelt werden. (Schwache Empfehlung, Evidenzlevel 2c).

- Der Analfistelplug kann zur Behandlung bei komplexen Fisteln eingesetzt werden. (Schwache Empfehlung, Evidenzlevel 2c).

- Endoanale Verschiebelappenplastiken können zur Behandlung bei komplexen Fisteln eingesetzt werden. (Starke Empfehlung, Evidenzlevel 1c).

- Komplexe Fisteln können durch Fadeneinlage und sequenzierte Fistelspaltung behandelt werden. (Starke Empfehlung, Evidenzlevel 1b). - Komplexe Fisteln können durch die LIFT-Methode behandelt werden. (Keine Empfehlung).

Die Aufarbeitung der Empfehlungen der Britisch-Irischen Gesellschaft für Koloproktologie aus dem Jahr 2007 [483] muss nach 9 Jahren als nicht mehr aktuell angesehen werden.

Diese Empfehlungen müssen jedoch unter Berücksichtigung der aktuellen Literatur revidiert werden. Gerade für die Fadentechniken wurden in Studien [46,
166] und Reviews [345] hohe Inkontinenzraten beschrieben. Aktuelle Ergebnisse zum Fibrinkleber bieten geringe Erfolgsraten. Die Heilungsrate nach Fistelplug sinkt mit Zunahme der Publikationen. Aktuelle Reviews stellen somit bei fehlenden validen Daten nur eine eingeschränkte Hilfe für die Erstellung von Therapierichtlinien dar.

Aktuell sind die Leitlinien aus Italien [21], die in einem Konsensuspapier 2015 veröffentlicht wurden. Interessanterweise fehlt in der Publikation ein Verweis auf die umfangreiche Deutsche S3-Leitlinie, die auch in englischer Sprache publiziert wurde. Es wird zwischen einfachen und komplexen Fisteln unterschieden:

\section{Einfache Fisteln.}

1. Eine primäre Spaltung ist sinnvoll. (Evidenzgrad 1B)

2. Die Verwendung eines elektrischen Messers verkürzt die Heilungsdauer und verursacht weniger Schmerzen. (Evidenzgrad 2B)

3. Eine Marsupialisation verkürzt die Heilungsdauer. (Evidenzgrad 1B)

4. Fadentechniken sollten wegen einer längeren Heilungsdauer und vermehrten Schmerzen nicht angewendet werden. (Evidenzgrad 1B)

5. Der Fistelspaltung ist gegenüber der kompletten Fistelexzision der Vorzug zu geben. (Evidenzgrad $1 B$ )

6. Neue Techniken (LIFT, VAAFT, FiLaC) sind mögliche Therapieoptionen. (Evidenzgrad 1B)

\section{Komplexe Fisteln.}

1. Die Flap-Technik stellt eine mögliche Therapieoption dar (Heilungsrate: $70 \%)$. (Evidenzgrad 1B)

2. Die Flap-Technik hat einen geringen bis moderaten Einfluss auf die Kontinenzleistung. (Evidenzgrad 1B)

3. Weitere mögliche Therapieoptionen sind: LIFT-Verfahren (Evidenzgrad 1B), Kürettage und Fibrinkleber (Evidenzgrad 1B), autologe Stammzellen mit/ohne Fibrinkleber (Evidenzgrad 2B), Kollagen (Evidenzgrad 2B), Fistelplug (Evidenzgrad 1C), VAAFT (Evidenzgrad 2C), Laser mit/ohne Verschluss des inneren Fistelostiums (Evidenzgrad 2C). 
Kritisch muss hier angemerkt werden, dass im Prinzip alle zur Verfügung stehenden Verfahren als sinnvoll angesehen werden, was derzeit aufgrund der Literaturlage auch kaum anders möglich ist. Aus Sicht der Autoren konnten die beiden randomisierten Studien eher keinen Vorteil für eine Marsupialisation ergeben. Der Einsatz von LIFT, VAAFT und FiLaC bei oberflächlichen Fisteln sollte sehr kritisch gesehen werden.

Aktuell wurde im Auftrag der Europäischen Gesellschaft für Koloproktologie ein Review der vorliegenden Leitlinien vorgelegt [91]. Auch die erste Version dieser Leitlinie wird aufgeführt. Letztlich decken sich die Aussagen weitgehend mit denen dieser Leitlinie:

\section{Diagnostik.}

- Magnetresonanztomographie wird empfohlen. (Konsens, Evidenzlevel 1a)

- Die Endosonographie kann in der Hand des erfahrenen Untersuchers wichtige Informationen geben. (Konsens, Evidenzlevel 1a)

- Die Narkoseuntersuchung hat einen wichtigen Stellenwert für Diagnostik und Klassifikation von Analfisteln. (Konsens, Evidenzlevel 1b)

- Die Computertomographie ist der MRT und der Endosonographie unterlegen. (Kein Konsens, Evidenzlevel 2b)

- Die Fistulographie spielt keine Rolle in der Diagnostik. (Konsens, Evidenzlevel 2b)

\section{Klassifikation.}

- Es existiert keine allgemein akzeptierte Klassifikation von Analfisteln. (Kein Konsens, Evidenzlevel 3b)

- Es existiert keine klare Definition der Begriffe „einfache“ und „komplexe“ Analfistel. (Kein Konsens, Evidenzlevel 3b)

\section{Chirurgie.}

- Die Fistelspaltung spielt eine wichtige Rolle bei der Behandlung von Analfisteln (Konsens, Evidenzlevel 1b). Sie sollte bei hohen Analfisteln eher nicht zur Anwendung kommen. (Konsens, Evidenzlevel 3a)

- Die Verwendung eines schneidenden Fadens wird wegen des Inkonti- nenzrisikos nicht empfohlen. (Kein Konsens, Evidenzlevel 2a)

- Die Einlage eines losen Fadens ist eine effektive Maßnahme um einen Rezidivabszess zu verhindern (Konsens, Evidenzlevel 2a). Sie stellt eine wichtige Alternativtherapie bei komplexen Analfisteln dar. (Konsens, Evidenzlevel 2a)

- Einige Chirurgen bevorzugen die Einlage eines losen Fadens vor definitiver Therapie. (Kein Konsens, Evidenzlevel 2a)

- Verschiebelappenplastiken stellen eine oft benutzte Therapie bei Analfisteln dar. (Konsens, Evidenzlevel 1b)

- Fibrinkleber stellt eine Behandlungsoption bei komplexen Analfisteln dar, obwohl die Effektivität unklar ist (Konsens, Evidenzlevel 1b). Es ist unklar, welche Patienten von dieser Behandlung profitieren. (Konsens, Evidenzlevel 1b)

- Der Fistelplug stellt eine erprobte Therapieoption bei Analfisteln dar. (Konsens, Evidenzlevel 1b)

- Die LIFT-Methode stellt eine Behandlungsoption bei ausgewählten Patienten dar. (Kein Konsens, Evidenzlevel 4)

\section{Perioperatives Management}

\section{Narkose}

Analfisteloperationen können sowohl in Allgemeinanästhesie als auch in regionaler Anästhesie durchgeführt werden. Eine Lokalanästhesie ist nicht sinnvoll. Ein perianaler Block wurde jedoch als gute Option beschrieben [363].

- Evidenzlevel: KKP (klinischer Konsensuspunkt)

- Konsensusstärke: starker Konsens

\section{Darmvorbereitung}

Kontrovers wird die Vorbehandlung bei komplexen Analeingriffen diskutiert. Während in der Kolonchirurgie keine Vorteile für die präoperative Darmreinigung gesehen werden konnten [158], wird bei komplexen Analeingriffen von den meisten Chirurgen eine präoperative Darmvorbereitung durchgeführt. Nessim et al. [283] vergleichen in ei- ner randomisierten Studie flüssige Kost in Verbindung mit Motilitätshemmern (Loperamid) mit normaler Kost nach verschiedenen rekonstruktiven Analeingriffen. Bei gleichen klinischen und funktionellen Ergebnissen nach 13 Monaten verweisen sie auf die höheren Kosten für die Spezialkost. In einer prospektiv-randomisierten Studie konnten Joos et al. [201] im Vergleich zwischen parenteraler und enteraler Ernährung mittels voll resorbierbarer Kost Vorteile für die enterale Ernährung nachweisen. Bei beiden Methoden wird jedoch der frühe postoperative Stuhlabgang unterdrückt. Ziel aller Maßnahmen ist die normale Stuhlentleerung unter Vermeiden stärkeren Pressens mit Gefährdung der angelegten Nähte.

Fazit für die Praxis. Bei der Analfistelspaltung oder Fadeneinlage ist keine spezielle Darmvorbereitung oder postoperative Behandlung erforderlich. $\mathrm{Ob}$ eine präoperative Darmreinigung und/ oder die Verzögerung der Stuhlentleerung nach der Operation die Heilungsraten bei plastischen Therapieverfahren beeinflussen können, ist derzeit ungeklärt.

- Evidenzlevel: 5

- Empfehlungsgrad: 0

- Konsensusstärke: Konsens

\section{Reinigung und Wundversorgung nach Analchirurgie}

Die postoperative Nachsorge nach Analchirurgie ist fast immer unproblematisch. Die äußere anale Wunde heilt planmäßig stets sekundär. Durch regelmäßiges Austasten der Wunde können Sekretverhalte in der Tiefe vermieden werden. Insbesondere während der Heilungsphase sollte Verstopfung vermieden und darauf geachtet werden, dass der Stuhl weich ist.

Die Reinigung der äußeren Analregion erfolgt in aller Regel durch Ausduschen mit Wasser von Trinkwasserqualität. Für die Reinigung der Analregion sind bei der Routineversorgung weitere oder spezielle Maßnahmen aus Sicht der Autoren dieser Leitlinie weder erforderlich noch üblich, auch existieren keine klinischen Studien zur Behandlung von 
beabsichtigt offen bzw. sekundär heilenden analen Wunden mit Leitungswasser.

Bereits seit Längerem wird diskutiert, ob Lösungen zur postoperativen Reinigung der Analregion den Anspruch auf Sterilität erfüllen müssen. Untersuchungen deuten darauf hin, dass Leitungswasser von Trinkwasserqualität ebenso effektiv in der Vermeidung von Wundinfektionen sein kann wie die Reinigung und Spülung mit physiologischer Kochsalzoder Ringerlösung [47]. Eine endgültige Bewertung von Lösungen zur Wundspülung ist aufgrund des fehlenden klinischen Vergleichs mit einheitlichem Studiendesign jedoch zurzeit nicht möglich. Aus der proktologischen Erfahrung ist bekannt, dass für den Dekontaminationserfolg gerade auch die Menge der Spüllösung entscheidend sein dürfte. Dies kann zur Erklärung dienen, warum bei der Anwendung von Trinkwasser regelmäßig klinisch günstige Resultate erzielt werden [192].

Ungeachtet dessen gilt, dass zum Spülen steril zu versorgender Wunden einschließlich infizierter, planmäßig jedoch primär heilender Wunden nur sterile Lösungen verwendet werden [347], z. B. sterile Kochsalz-, Ringer-Laktat-Lösung, physiologische BSS(„,balanced salt solution“)- beziehungsweise PBS(„phosphatebuffered saline“)-Lösungen oder sterilgefiltertes Wasser (über endständige Sterilfilter am Wasserauslass).

In mehreren Studien wird das Rauchen als negativer Faktor für den Erfolg in der plastischen Fistelchirurgie herausgearbeitet [121, 501]. Zimmermann et al. [501] verweisen dabei auf eine Minderung der Schleimhautdurchblutung durch das Rauchen. Auch in anderen Studien konnte das Rauchen als Risikofaktor für den operativen Erfolg nachgewiesen werden [146]. Auch Adipositas wurde als negativer Faktor für den Heilungsverlauf beschrieben [372].

Fazit für die Praxis. Es ist Sorge $\mathrm{zu}$ tragen, dass sich die äußere Drainageöffnung nicht vorzeitig verschließt (Schnittführung, Austasten der Wunde). Der Analbereich soll regelmäßig durch Spülung gereinigt werden. Rauchen konnte als klarer Risikofaktor für eine gestörte Wundheilung identifiziert werden.

- Evidenzlevel: 4

- Empfehlungsgrad: B (Begründung: Aus ethischen Gründen ist eine Überprüfung dieser allgemein akzeptierten Aussage durch randomisierte Studien nicht möglich.)

- Konsensusstärke: starker Konsens

\section{Antibiotikatherapie}

Auch der Stellenwert einer perioperativen Antibiotikatherapie ist bisher ungeklärt. Lediglich eine Studie randomisiert die Flap-Technik mit und ohnelokale Antibiose mit Gentamycin und kann keinen Vorteil für die Antibiotikatherapie nachweisen [163].

Fazit für die Praxis. Der Stellenwert einer perioperativen Antibiotikatherapie ist ungeklärt.

- Evidenzlevel: 5

- Empfehlungsgrad: 0

- Konsensusstärke: Konsens

\section{Stomaanlage}

Eine Stomaanlage im Rahmen der Analfistelchirurgie ist nur selten erforderlich. Die Indikation besteht in erster Linie bei ausgeprägter Destruktion des Analkanals bei daraus resultierender Stuhlinkontinenz. In einer Zusammenstellung von 355 Patienten mit hohen trans- und suprasphinktären Fisteln war lediglich bei 3 Patienten eine Stomaanlage erforderlich, wobei bei einem wegen einer persistierenden Fistel und Inkontinenz ein definitives Stoma angelegt wurde [289].

Fazit für die Praxis. Eine Stomaanlage ist nur in Ausnahmefällen indiziert.

- Evidenzlevel: 5

- Empfehlungsgrad: 0

- Konsensusstärke: Konsens

\section{Komplikationen}

\section{Perioperative Komplikationen}

Komplikationen nach Analfisteleingriffen unterscheiden sich grundsätzlich nicht von anderen Analeingriffen. Im unmittelbaren perioperativen Verlauf sind dabei die lokale Nachblutung, die in einigen Fällen operativ gestillt werden muss, und der Harnverhalt zu erwähnen. Dieser stellt keine prozedurspezifische Komplikation dar und tritt häufiger nach Hämorrhoidenoperationen auf. Risikofaktoren für den postoperativen Harnverhalt sind dabei insbesondere die unzureichende Schmerztherapie und die $\mathrm{zu}$ hohe intraoperative intravenöse Flüssigkeitszufuhr [434, 489]. Bei plastischen Fistelrekonstruktionen besteht die Gefahr der lokalen Infektion mit sekundärer Dehiszenz der angelegten Nähte. In den meisten Fällen ist die Nahtdehiszenz mit einer Persistenz der Fistel assoziiert.

\section{Kontinenzstörungen nach Analfisteloperationen}

Die Kontinenzstörung stellt, wie bereits im Vorhergehenden mehrfach ausgeführt, eine häufige Komplikation nach Analfisteloperationen dar und ist i. d. R. multifaktoriell bedingt, wobei Sphinkterläsionen im Vordergrund stehen [90].

Bereits die Exposition des Analkanals mittels Spreizer kann durch die Zerreißung von Schließmuskelanteilen Kontinenzveränderungen hervorrufen, wobei asymptomatische Läsionen wesentlich häufiger als klinische Störungen beobachtet wurden $[182,184,460]$. Eine prospektiv-randomisierte Studie [500] vergleicht die Dilatation des Analkanals mittels Parks-Retraktor und die Eventeration des Anoderms mittels Scott-Retraktor im Rahmen der Fisteloperation. Sie findet eine deutliche Reduktion des Inkontinenzscores nach Verwendung des Parks-Retraktors, möglicherweise durch eine Internusschädigung. Endosonographische Untersuchungen zeigen nach Analdilatation Internusläsionen bei $76 \%$ und Externusläsionen bei $24 \%$ der Untersuchten [404]. Nach plastischem Fistelverschluss wiesen $57 \%$ eine Internus- und $29 \%$ eine Externusläsion auf. Von den Patienten mit Externusläsionen bestand jedoch bei $62 \%$ keine Kontinenzstörung und nach reiner Internusläsion war bei $75 \%$ die Kontinenz regelrecht. Deutlich negativ wirkten sich wiederholte Analeingriffe aus: Nach zwei und mehr Eingriffen wurden Internus- 
läsionen bei $74 \%$ und Externusläsionen bei $30 \%$ endosonographisch nachgewiesen; $26 \%$ der Untersuchten berichteten über Störungen der Kontinenz.

Manometrische Studien beobachten einen signifikanten Druckabfall nach Analfisteloperationen. Belliveau et al. [37] und Sainio et al. [359] beschreiben eine signifikante Erniedrigung der Manometriewerte nach Spaltung interund transsphinktärer Fisteln mit entsprechenden Kontinenzstörungen. Eine wichtige Rolle spielt hierbei auch die Verkürzung des Analkanals, die nach Spaltung transsphinktärer Fisteln ausgeprägter ist als bei intersphinktären Fisteln. Die Bedeutung der Analkanallänge wird von Lunniss herausgearbeitet [244]. Eine neuere Studie von Gustafsson et al. [162] zeigt auch nach plastischen Operationsverfahren eine Minderung der Manometriewerte 3 und 12 Monate postoperativ.

Einen Einfluss auf die terminale Latenzzeit des Nervus pudendus wurde in einer Studie aus dem Jahr 2006 ausgeschlossen [90].

Die Dicke des Externusanteils zwischen Fistel und Anoderm bei als transsphinktär klassifizierten Fisteln zeigt eine große Variationsbreite. Cavanaugh et al. [59] beobachteten, dass bei $70 \%$ von 62 untersuchten Patienten der Externusanteilunter $25 \%$ lag. Ein Anteilvon 26-50\% wurde bei $22 \%$, einer von $51-75 \%$ bei $6 \%$ und letztlich ein Anteil von mehr als $75 \%$ lediglich bei $2 \%$ gesehen.

Die Korrelation zwischen durchtrennten Sphinkteranteilen und Störungen der Kontinenz wird von Garcia-Aguilar et al. [134] untersucht. Nach Fistelspaltung ohne relevante Durchtrennung von Externusanteilen berichteten bereits $38 \%$ der Patienten (52 von 138) über Kontinenzstörungen. Dieser Anteil erhöhte sich erwartungsgemäß mit der Menge der durchtrennten Muskelmasse: $<25 \%$ : $44 \%$ (17 von 39 ); $26-50 \%: 60 \%$ (18 von 30 ); $51-75 \%: 55 \%$ (6 von 11 ) und $>75 \%$ : $75 \%$ (12 von 16). Auch die schrittweise Durchtrennung des Sphinkters mittels schneidenden Fadens führt zu einer hohen Inkontinenzrate, wie Ritchie et al. [345] in einem Review herausarbeiten konnten.
Da die bewusste Durchtrennung von Muskelanteilen oft unverzichtbarer Bestandteil des operativen Vorgehens ist, stellen Analfisteloperationen eine häufige Ursache von Kontinenzstörungen dar. Daraus erklärt sich eine hohe Rate von Kontinenzstörungen zwischen 20 und $64 \%$ bei der Fistelspaltung. Bei den plastischen Verfahren findet sich ebenfalls eine weite Streubreite der Inkontinenzrate von 0-42\%, ohne dass ein Unterschied zwischen den verschiedenen plastischen Verfahren (Mukosa/ Submukosa-Flap, Rectal-AdvancementFlap und Anoderm-Flap) auszumachen ist [217]. Diese Daten sind sowohl auf die unterschiedliche Definition des Begriffs Inkontinenz durch die Untersucher, als auch auf die Komplexität der Fisteln und Voroperationen mit bereits erfolgter Schließmuskeldurchtrennung zurückzuführen. Das Ausmaß der Kontinenzstörung steigt mit der Genauigkeit der klinischen Erfassung. So berichteten Cavanaugh et al. [59], die die Inkontinenz anhand eines Scores erfassten, mit 64 \% über die höchste Rate an Kontinenzstörungen nach Fistelspaltung. Andererseits beschreibt eine aktuelle Studie [131] bei Patienten ohne Risikofaktoren nach Durchtrennung der unteren zwei Drittel des Externus eine hohe Heilungsrate ohne nennenswertes Inkontinenzrisiko nach Spaltung.

Weitere aktuelle Arbeiten bestätigen die Veränderung der anorektalen Morphologie und Minderung der Kontinenz durch Eingriffe sowohl bei oberflächlichen [435] als auch bei hohen Analfisteln [348]. Kontinenzstörungen können sich auch noch nach Jahren durch eine Summierung von Vorschäden und Alterungsvorgängen manifestieren. Dies ist insbesondere bei der Auswertung der Literatur zu berücksichtigen, da hier oft nur ein kurzes Follow-up beobachtet wird.

Durch die Komplexität des Krankheitsbilds sind oft wiederholte Eingriffe erforderlich, die jeweils mit Sphinkterschädigungen verbunden sein können. In der Studie von Zimmermann et al. [502] bestand bereits bei $52 \%$ der Patienten präoperativ eine Beeinträchtigung der Kontinenzleistung. Die hohe Variabilität der Inkontinenzraten in den verschiedenen Studien erklärt sich durch die Heterogenität der Patientenkollektive mit Einschluss verschiedener Fisteltypen (Typ II und III nach Parks, rektovaginale Fisteln), Fisteln bei chronischentzündlichen Darmerkrankungen und unterschiedliche Zahlen an Voroperationen [217]. Weiterhin sind bei der Auswertung der Arbeiten die höchst unterschiedlichen Nachuntersuchungsquoten zwischen 40 und $90 \%$ zu berücksichtigen $[134,173,458]$. Neben dem Fistelrezidiv mit der Notwendigkeit wiederholter Eingriffe beeinträchtigt insbesondere die Minderung der Kontinenzleistung die Zufriedenheit und die Lebensqualität [135, 379]. Eine Aufarbeitung historischer Krankenunterlagen von Blumetti et al. [45] zeigte eine deutliche Abkehr von Spaltungsverfahren hin zu sphinkterschonenden Verfahren. So wurde im Zeitraum 1975-1979 bei $99 \%$ und 1985-1989 bei $92 \%$ aller Fisteln eine Spaltung durchgeführt. Im Zeitraum 1995-2000 lag die Rate bei $69 \%$ und sank 2005-2010 auf 51 \%. Auch weitere Studien bestätigen den Stellenwert des Kontinenzerhalts für die Patientenzufriedenheit [119].

Risikofaktoren für eine Kontinenzstörung nach Fisteloperationen sind: weibliches Geschlecht, Alter >50 Jahre, hohe (trans-/suprasphinktäre) Analfisteln, Art und Anzahl der vorhergehenden Operationen [134, 293]. Diese sollten bei der Wahl des Operationsverfahrens berücksichtigt werden. Das erhöhte Risiko bei Frauen erklärt sich neben möglichen geburtshilflichen Schädigungen aus der speziellen Sphinktermorphologie, nach welcher der weibliche Schließmuskel ventral schwächer als beim Mann ausgebildet ist [410, 474].

Diese Faktoren müssen insbesondere vor dem Hintergrund einer zunehmenden Informationsvermittlung durch das Internet [378] bei der Wahl des Operationsverfahrens berücksichtigt und mit dem Patienten/der Patientin besprochen werden. Für die neueren Verfahren werden zwar deutlich niedrigere Inkontinenzraten beschrieben, sie sind überwiegend aber auch mit einer deutlich niedrigeren Heilungsrate assoziiert.

Fazit für die Praxis. Die wichtigste Komplikation nach Analfisteloperationen be- 
steht in der Minderung der Stuhlkontinenzleistung. Die Anzahl der Patienten mit Kontinenzstörungen steigt mit dem Ausmaß der operativen Intervention, der Zahl der Voroperationen bzw. Vorschädigungen (Entbindung) und der Menge an durchtrennter Muskelmasse. Frauen und ältere Patienten weisen ein höheres Risiko auf. Vor diesem Hintergrund sollte immer eine umfangreiche Patientenaufklärung erfolgen. Eine bestmögliche Schonung des Sphinkterapparats ist $\mathrm{zu}$ fordern, wobei bei wiederholtem Rezidiv die Option der definitiven Heilung gegen die mögliche Minderung der Kontinenzleistung abzuwägen ist.

- Evidenzlevel: 1c

- Empfehlungsgrad: A

- Konsensusstärke: starker Konsens

\section{Maligne Entartung}

Malignome auf dem Boden einer Analfistel sind sehr selten. Demzufolge wird die Indikation zu einer routinemäßigen Untersuchung des Resektats unterschiedlich gesehen [254, 481]. Andererseits wird die maligne Entartung von perinealen Fisteln in der Literatur in vielen Fallbeispielen beschrieben $[63,125,307,392$, 486]. Gaertner et al. [129] berichten über 14 Patienten. Die Dauer der Anamnese umfasste den Zeitraum von weniger als einem Jahr bis zu 60 Jahren. Überwiegend handelte es sich um komplexe transsphinktäre oder rektovaginale Fisteln, die alle bereits voroperiert worden waren. In weniger als der Hälfte der Patienten war der Malignitätsverdacht im Rahmen der klinischen Untersuchung aufgefallen. Bei nahezu allen dokumentierten Adenokarzinomen lag ein lokal fortgeschrittenes Tumorstadium vor. Durch eine abdominoperineale Resektion, ggf. in Kombination mit einer neoadjuvanten Radiochemotherapie, konnten 10 von 14 Patienten geheilt werden. Als Sonderfall beschreiben Rollinson et al. [353] die Metastase eines Sigmakarzinoms in einer perinealen Fistel. Neuere relevante Publikationen zu diesem Thema konnten nicht identifiziert werden.

Fazit für die Praxis. Die Entstehung eines Malignoms in einer chronischen Analfistel ist selten, aber möglich. Aus diesem
Grunde ist eine histologische Untersuchung des Resektats zu empfehlen.

- Evidenzlevel: 5

- Empfehlungsgrad: 0

- Konsensus: starker Konsens

\section{Zusammenfassung}

\section{Evidenzbasierte Behandlung der kryptoglandulären Analfistel}

1. Kryptoglanduläre Analfisteln sind eine relativ häufige Erkrankung mit einem Häufigkeitsgipfel bei jungen männlichen Erwachsenen.

2. Die Klassifikation der Analfisteln orientiert sich nach der Beziehung des Fistelgangs zum Schließmuskel (Typ I bis III nach Parks). Die von Parks beschriebenen Typ-IVFisteln sowie die subanodermal verlaufenden Fisteln sind i. d. R. nicht kryptoglandulärer Genese.

3. In der Diagnostik sind die Anamnese und die klinische Untersuchung für die Operationsindikation als ausreichend anzusehen. Zusätzlich soll intraoperativ eine Sondierung und/oder Anfärbung des Fistelgangs erfolgen. Endosonographie und $\mathrm{Ma}$ gnetresonanztomographie sind in ihrer Aussage weitgehend gleichwertig und bei komplexen bzw. klinisch nicht sicher zu klassifizierenden Fisteln sowie bei Abszessen als ergänzende Untersuchung einzusetzen. (Evidenzlevel 1a, Empfehlungsgrad A)

4. Die Therapie der Analfistel ist prinzipiell eine operative. Es stehen 6 erprobte Verfahren zur Verfügung:

a) Fistelspaltung,

b) Fadendrainage,

c) Exzision und Verschluss des inneren Ostiums mittels Lappenplastik, d)plastische Rekonstruktion mit Sphinkternaht,

e) LIFT-Methode,

f) Okklusion mit Biomaterialien.

5. Eine Spaltung sollte nur bei oberflächlichen Fisteln erfolgen. Die Gefahr der postoperativen Kontinenzstörung steigt mit der Menge des durchtrennten Sphinkteranteils. (Evidenzlevel 2b; Empfehlungsgrad B)

6. Bei allen hohen Analfisteln soll ein sphinkterschonendes Verfahren zur
Anwendung kommen. Die Ergebnisse der verschiedenen Techniken (FlapTechnik, LIFT, Spaltung und Rekonstruktion) sind weitgehend identisch. Für die Okklusion durch Biomaterialien (Fibrinkleber, Kollagen, autologe Stammzellen, Fistelplug) ist insgesamt eine deutlich geringere Heilungsrate zu konstatieren. Für die neueren technischen Verfahren (Laseranwendung, VAAFT, OTSCClip) konnte noch kein eindeutiger Vorteil gegenüber den herkömmlichen Verfahren dargestellt werden. (Evidenzlevel 1b; Empfehlungsgrad A)

7. Jede Behandlung einer Analfistel ist grundsätzlich mit dem Risiko einer Kontinenzminderung verbunden. Neben der bewussten Durchtrennung von Schließmuskelanteilen spielen hier auch Vorschäden, Voroperationen und weitere Faktoren (Alter, Geschlecht u. a.) eine Rolle. Ein hohes Risiko einer Kontinenzstörung besteht nach Spaltung hoher Analfisteln. Die geringere Heilungsrate der Verfahren mit Okklusion des Fistelkanals ist mit einer geringeren Inkontinenzrate vergesellschaftet. (Evidenzlevel 1c; Empfehlungsgrad A)

\section{Korrespondenzadresse}

\section{Dr. A. Ommer}

End- und Dickdarmdarm-Zentrum Essen Rüttenscheider Straße 66, 45130 Essen, Deutschland aommer@online.de

\section{Einhaltung ethischer Richtlinien}

\begin{abstract}
Interessenkonflikt. Eine ausführliche Liste von möglichen Interessenkonflikten steht Ihnen online im elektronischen Volltextarchiv auf SpringerMedizin.de unterhttp://www.springermedizin.de/der-chirurgzur Verfügung. Sie finden die Liste unter „Supplementary Material".
\end{abstract}

Dieser Beitrag beinhaltet keine von den Autoren durchgeführten Studien an Menschen oder Tieren.

\section{Literatur}

1. A ba-bai-ke-re M, Wen H et al (2014) Randomized controlled trial of minimally invasive suregry using acellular dermal matrix for complex anorectal fistula. World J Gastroenterol 16(26):3279-3285 
2. Abbas MA, Lemus-Rangel $R$ et al (2008) Longterm outcome of endorectal advancement flap for complex anorectal fistulae. Am Surg 74(10):921-924

3. Abbas MA, Tejirian T (2008) Bioglue for the treatment of anal fistula is associated with acute anal sepsis. Dis Colon Rectum 51(7):1155 (author reply 1156)

4. Abcarian AM, Estrada JJ et al (2012) Ligation of intersphincteric fistula tract: early results of a pilot study. Dis Colon Rectum 55(7):778-782

5. Abcarian H, Dodi G et al (1987) Fistula-in-ano. Int J Colorectal Dis 2(2):51-71

6. Abel ME, Chiu YS et al (1993) Autologous fibrin glue in the treatment of rectovaginal and complex fistulas. Dis Colon Rectum 36(5):447-449

7. Abeysuriya V, Salgado LS et al (2010) The distribution of the anal glands and the variable regional occurrence of fistula-in-ano: is there a relationship? Tech Coloproctol 14:317-321

8. Aboulian A, Kaji AH et al (2011) Early result of ligation of the intersphincteric fistula tract for fistula-in-ano. Dis Colon Rectum 54(3):289-292

9. Abou-Zeid AA (2011) Anal fistula: intraoperative difficulties and unexpected findings. World J Gastroenterol 17(28):3272-3276

10. AckermannC, Tondelli Petal (1994) Sphinkterschonende Operation der transsphinkteren Analfistel. Schweiz Med Wochenschr 124(27-28):1253-1256

11. Adamina M, Hoch JS et al (2010) To plug or not to plug: a cost-effectiveness analysis for complexanal fistula. Surgery 147(1):72-78

12. Adamina M, Ross T et al (2014) Anal fistula plug: a prospective evaluation of success, continence and quality of life in the treatment of complex fistulae. Colorectal Dis 16(7):547-554

13. Adamo K, Sandblom G et al (2016) Prevalence and recurrence rate of perianal abscess-a populationbased study, Sweden 1997-2009. Int J Colorectal Dis 31(3):669-673

14. AdamsT, Yang Jetal(2008) Long-term outlookafter successful fibrin glue ablation of cryptoglandular transsphincteric fistula-in-ano. Dis Colon Rectum 51(10):1488-1490

15. Aguilar PS, Plasencia G et al (1985) Mucosal advancement in the treatment of anal fistula. Dis Colon Rectum 28(7):496-498

16. Aitola P, Hiltunen KM et al (1999) Fibrin glue in perianal fistulas - a pilot study. Ann Chir Gynaecol 88(2):136-138

17. Akovbiantz A, Arma S et al (1968) Kontinenz nach Sphinkterspaltung bei Analfissuren und Analfisteln. Helv Chir Acta 35(1):260-265

18. AlasariS, Kim NK(2014) Overview of analfistula and systematic review of ligation of the intersphincteric fistula tract (LIFT). Tech Coloproctol 18(1):13-22

19. Alexander SM, Mitalas LE et al (2008) Obliteration of the fistulous tract with BioGlue(R) adversely affects the outcome of transanal advancement flap repair. Tech Coloproctol 12(3):225-228

20. Altomare DF, Greco VJ et al (2010) Seton or glue for trans-sphincteric anal fistulae: a prospective randomized crossover clinical trial. Colorectal Dis 13(1):82-86

21. Amato A, Bottini C et al (2015) Evaluation and management of perianal abscess and anal fistula: a consensus statement developed by the Italian Society of Colorectal Surgery (SICCR). Tech Coloproctol 19(10):595-606

22. Amin SN, Tierney GM etal (2003) V-Y advancement flap for treatment of fistula-in-ano. Dis Colon Rectum 46(4):540-543

23. Arroyo A, Perez-Legaz J et al (2012) Fistulotomy and sphincter reconstruction in the treatment of complex fistula-in-ano: long-term clinical and manometric results. Ann Surg 255(5):935-939

24. Athanasiadis S, Girona J (1983) Neue Behandlungsmethoden der perinealen Fisteln bei Morbus Crohn. Langenbecks Arch Chir 360:119-132

25. Athanasiadis S, Helmes C et al (2004) The direct closure of the internal fistula opening without advancement flap for transsphincteric fistulas-in ano. Dis Colon Rectum 47(7):1174-1180

26. Athanasiadis S, Köhler A et al (1994) Treatment of high anal fistulae by primary occlusion of the internal ostium, drainage of the intersphincteric space, and mucosal advancement flap. Int J Colorectal Dis 9(3):153-157

27. Athanasiadis S, Nafe M et al (1995) Transanaler rektaler Verschiebelappen (rectal advancement flap) versus Mucosaflap mit Internusnaht im Management komplizierter Fisteln des Anorectums. Langenbecks Arch Chir 380(1):31-36

28. Atkin GK, Martins J et al (2011) Die Fistulotomie - Eine gute Option bei hohen Analfisteln. Coloproctology 33(6):345-352

29. Atkin GK, Martins J et al (2011) For many high anal fistulas, lay open is still a good option. Tech Coloproctol 15(2):143-150

30. Attaallah W, Tuney D et al (2014) Should we consider topical silver nitrate irrigation as a definitive nonsurgical treatment for perianal fistula? Dis Colon Rectum 57(7):882-887

31. Banasiewicz T, Hermann J et al (2014) Sandwich technique' with bridging, a modification of negative pressure wound therapy for anal fistulas. Tech Coloproctol 19(3):173-175

32. Barillari P, Basso Let al (2006) Cyanoacrylate glue in the treatment of ano-rectal fistulas. Int J Colorectal Dis 21(8):791-794

33. Barker JA, Conway AM et al (2011) Supralevator fistula-in-ano in tuberculosis. Colorectal Dis 13(2):210-214

34. Beaulieu R, Bonekamp Detal (2013) Fistula-in-ano: when to cut, tie, plug, or sew. J Gastrointest Surg 17(6):1143-1152

35. Becker A, Koltun L et al (2006) Simple clinical examination predicts complexity of perianal fistula. Colorectal Dis 8(7):601-604

36. Beets-Tan RG, Beets GL et al (2001) Preoperative MR imaging of anal fistulas: Does it really help the surgeon? Radiology 218(1):75-84

37. Belliveau P, Thomson JP et al (1983) Fistula-inano. A manometric study. Dis Colon Rectum 26(3):152-154

38. Belmonte Montes C, Ruiz Galindo GH et al (1999) Fistulotomy vs fistulectomy. Ultrasonographic evaluation of lesion of the anal sphincter function. Rev Gastroenterol Mex 64(4):167-170

39. Benjelloun EB, Souiki T et al (2014) Endoanal ultrasound in anal fistulas. Is there any influence on postoperative outcome? Tech Coloproctol 18(4):405-406

40. BenliceC, Yildiz Metal (2015) Fistula tract curettage and the use of biological dermal plugs improve high transsphincteric fistula healing in an animal model. Int J Colorectal Dis 31(2):291-299

41. Beynon J, Carr N (1988) Master John of Arderne surgeon of Newark.J R Soc Med 81(1):43-44

42. Bleier Jl, Moloo H et al (2010) Ligation of the intersphincteric fistula tract: an effective new technique for complex fistulas. Dis Colon Rectum 53(1):43-46

43. Blom J, Husberg-Sellberg B et al (2014) Results of collagen plug occlusion of anal fistula: a multicentre study of 126 patients. Colorectal Dis 16(8):626-630
44. Blom J, Nystrom PO et al (2012) Endoana ultrasonography may distinguish Crohn's anal fistulae from cryptoglandular fistulae in patients with Crohn's disease: a cross-sectional study. Tech Coloproctol 15(3):327-330

45. Blumetti J, Abcarian A et al (2012) Evolution of treatment of fistula in Ano. World J Surg 36(5):1162-1167

46. Bokhari S, Lindsey I (2010) Incontinence following sphincter division for treatment of anal fistula. Colorectal Dis 12(7 Online):e135-e139

47. Browder LK, Sweet S et al (2009) Modified Hanley procedure for management of complex horseshoe fistulae. Tech Coloproctol 13(4):301-306

48. Brühl W, Neundorf G et al (1986) Das perineale Fistelleiden Teil B: Die langzeitige Fadendrainage. Coloproctology 3(8):175-181

49. Buchanan G, Halligan Set al (2002) Effect of MRI on clinical outcome of recurrent fistula-in-ano. Lancet 360(9346):1661-1662

50. Buchanan GN, Bartram Cl et al (2003) Efficacy of fibrin sealant in the management of complex anal fistula: a prospective trial. Dis Colon Rectum 46(9):1167-1174

51. Buchanan GN, Bartram Cl et al (2005) Value of hydrogen peroxide enhancement of threedimensional endoanal ultrasound in fistula-inano. Dis Colon Rectum 48(1):141-147

52. Buchanan GN, Halligan S et al (2004) Clinical examination, endosonography, and MR imaging in preoperative assessment of fistula in ano: comparison with outcome-based reference standard. Radiology 233(3):674-681

53. Buchanan GN, Owen HA et al (2004) Long-term outcome following loose-seton technique for external sphincter preservation in complex anal fistula. Br JSurg 91(4):476-480

54. Buchanan GN, Sibbons P et al (2005) Pilot study: fibrin sealant in anal fistula model. Dis Colon Rectum 48(3):532-539

55. Buchberg B, Masoomi H et al (2010) A tale of two (anal fistula) plugs: is there a difference in shortterm outcomes? Am Surg 76(10):1150-1153

56. BussenD, SailerMetal(2004)Wertigkeit deranalen Endosonographie in der Diagnostik anorektaler Fisteln. Zentralbl Chir 129(5):404-407

57. Cadeddu F, Salis F et al (2015) Complex anal fistula remains a challenge for colorectal surgeon. Int J Colorectal Dis 30(5):595-603

58. Campbell ML, Abboud EC et al (2013) Treatment of refractory perianal fistulas with ligation of the intersphincteric fistula tract: preliminary results. Am Surg 79(7):723-727

59. Cavanaugh M, Hyman N et al (2002) Fecal incontinence severity index after fistulotomy: a predictor of quality of life. Dis Colon Rectum 45(3):349-353

60. Champagne BJ, O'Connor LM et al (2006) Efficacy of anal fistula plug in closure of cryptoglandular fistulas: long-term follow-up. Dis Colon Rectum 49(12):1817-1821

61. Chan KM, Lau CW et al (2002) Preliminary results of using a commercial fibrin sealant in the treatment of fistula-in-ano. JR Coll Surg Edinb 47(1):407-410

62. Chan S, McCullough J et al (2012) Initial experience of treating anal fistula with the Surgisis anal fistula plug. Tech Coloproctol 16(3):201-206

63. Chandramohan K, Mathew AP et al (2010) Squamous cell carcinoma arising from longstanding perianal fistula. Int Wound J7(6):515-518

64. Chang SC, Lin JK (2003) Change in anal continence after surgery for intersphincteral anal fistula: a functional and manometric study. Int J Colorectal Dis 18(2):111-115 
65. Chapple KS, Spencer JA et al (2000) Prognostic value of magnetic resonance imaging in the management of fistula-in-ano. Dis Colon Rectum 43(4):511-516

66. Chen TA, Liu KY et al (2012) High ligation of the fistula track by lateral approach: a modified sphincter-saving technique for advanced anal fistulas. Colorectal Dis 14(9):e627-e630

67. Chew MH, Lee PJ et al (2013) Appraisal of the LIFT and BIOLIFT procedure: initial experience and short-term outcomes of 33 consecutive patients. Int JColorectal Dis 28(11):1489-1496

68. Choen S, Burnett S et al (1991) Comparison between anal endosonography and digital examination in the evaluation of anal fistulae. $\mathrm{Br}$ J Surg 78(4):445-447

69. Choi D, Sung Kim Het al (2010) Patient-performed seton irrigation for the treatment of deep horseshoefistula. Dis Colon Rectum 53(5):812-816

70. Chowbey PK, Khullar R et al (2013) Minimally Invasive Anal Fistula Treatment (MAFT) - an appraisal of early results in 416 Patients. Indian J Surg 77(Suppl 2):716-721

71. Christensen A, Nilas L et al (1986) Treatment of transsphincteric anal fistulas by the seton technique. Dis Colon Rectum 29(7):454-455

72. Christiansen J, Ronholt C (1995) Treatment of recurrent high anal fistula by total excision and primary sphincter reconstruction. Int J Colorectal Dis 10(4):207-209

73. Christoforidis D (2010) Who benefits from anal fistula plug. Dis Colon Rectum 53(8):1105-1106

74. Christoforidis D, Etzioni DA et al (2008) Treatment of complex anal fistulas with the collagen fistula plug. Dis Colon Rectum 51(10):1482-1487

75. Christoforidis D, Pieh MC et al (2009) Treatment of transsphincteric anal fistulas by endorectal advancement flap or collagen fistula plug: a comparative study. Dis Colon Rectum 52(1):18-22

76. Chuang-Wei C, Chang-Chieh W et al (2008) Cutting seton for complex anal fistulas. Surgeon 6(3):185-188

77. Chung W, Kazemi Petal (2009) Anal fistula plug and fibrin glue versus conventional treatment in repair of complex anal fistulas. Am J Surg 197(5):604-608

78. Cintron JR, Abcarian $\mathrm{H}$ et al (2013) Treatment of fistula-in-ano using a porcine small intestinal submucosa anal fistula plug. Tech Coloproctol 17(2):187-191

79. Cintron JR, Park JJ et al (1999) Repair of fistulas-inano using autologous fibrin tissue adhesive. Dis Colon Rectum 42(5):607-613

80. Cintron JR, Park JJ et al (2000) Repair of fistulasin-ano using fibrin adhesive: long-term followup. Dis Colon Rectum 43(7):944-949 (discussion 949-950)

81. Cioli VM, Gagliardi G et al (2015) Psychological stress in patients with anal fistula. Int J Colorectal Dis 30(8):1123-1129

82. Cirocchi R, Trastulli S et al (2013) The treatment of anal fistulas with biologically derived products: is innovation better than conventional surgical treatment? An update. Tech Coloproctol 17(3):259-273

83. Cirocco WC, Reilly JC (1992) Challenging the predictive accuracy of Goodsall's rule for anal fistulas. Dis Colon Rectum 35(6):537-542

84. Conole FD (1967) The significance of the anal gland in the pathogenesis of anorectal abszess and fistula. Am J Proctol 18(3):232-238

85. Coremans G, Dockx S et al (2003) Do anal fistulas in Crohn's disease behave differently and defy Goodsall's rule more frequently than fistulas that are cryptoglandular in origin? Am J Gastroentero 98(12):2732-2735

86. Corman ML (2008) The Surgisis AFP anal fistula plug: report of a consensus conference. Colorecta Dis 10(1):17-20

87. Cosman BC (1998) All's Well That Ends Well: Shakespeare's treatment of anal fistula. Dis Colon Rectum 41(7):914-924

88. CulpCE (1984) Use of Penrose drains to treat certain anal fistulas: a primary operative seton. Mayo Clin Proc59(9):613-617

89. D'Agostino G, D'Aloisio G et al (2000) Treatment of complex anal and rectovaginal fistulas using the endorectal mucosal flap technique. Minerva Chir 55(6):465-469

90. Daniel F, Thomas C et al (2006) Fistula in ano surgery has no impact on pudendal nerve terminal motor latency. Int J Colorectal Dis 21 (5):444-447

91. de Groof EJ, Cabral VN et al (2016) Systematic review of evidence and consensus on perianal fistula; an analysis of national and international guidelines. Colorectal Dis 18(4):0119-0134

92. de la Portilla F (2008) Evaluation of the use of BioGlue in the treatment of high anal fistulas: preliminary results of a pilot study: The Author Replies. Dis Colon Rectum 51:1156

93. de la Portilla F, Alba F et al (2013) Expanded allogeneic adipose-derived stem cells (eASCs) for the treatment of complex perianal fistula in Crohn's disease: results from a multicenter phase I/lla clinical trial. Int J Colorectal Dis 28(3):313-323

94. de la Portilla F, Rada R et al (2012) Evaluation of a new synthetic plug in the treatment of anal fistulas: results of a pilot study. Dis Colon Rectum 54(11):1419-1422

95. de la Portilla F, Rada R et al (2007) Evaluation of the use of BioGlue in the treatment of high anal fistulas: preliminary results of a pilot study. Dis Colon Rectum 50(2):218-222

96. de la Portilla F, Rada R et al (2010) Long-term Results Change Conclusions on BioGlue in the treatment of high anal fistulas. Dis Colon Rectum 53(8):1220-1221

97. de Oca J, Millan M et al (2011) Long-term results of surgery plus fibrin sealant for anal fistula. Colorectal Dis 14(1):e12-e15

98. de Parades V, Far HS et al (2010) Seton drainage and fibrin glue injection for complex anal fistulas. Colorectal Dis 12(5):459-463

99. de Parades V, Fathallah N et al (2012) Horseshoe tract of anal fistula: bad luck or an avoidable extension? Lessons from 82 cases. Colorectal Dis 14(12):1512-1515

100. de Parades V, Zeitoun JD et al (2010) Cryptoglandular anal fistula. JVisc Surg 147(4):203-215

101. Deshpande PJ, Sharma KR (1973) Treatment of fistula-in-ano by a new technique. Review and follow-up of 200 cases. Am J Proctol 24(1):49-60

102. Deshpande PJ, Sharma KR (1976) Successful nonoperative treatment of high rectal fistula. Am J Proctol 27(2):39-47

103. Dindo D, Weishaupt Det al (2008) Clinical and morphologic correlation after stapled transanal rectal resection for obstructed defecation syndrome. Dis Colon Rectum 51(12):1768-1774

104. Ding JH, Bi LX et al (2015) Impact of 3-dimensional endoanal ultrasound on the outcome of anal fistula surgery: a prospective cohort study. Colorectal Dis 17(12):1104-1112

105. Dixon M, Root J et al (2004) Endorectal flap advancement repair is an effective treatment for selected patients with anorectal fistulas. Am Surg 70(10):925-927
106. Doll D, Vassiliu P (2015) Silver nitrate for anal fistulas: a word of caution. Dis Colon Rectum 58(12): e459

107. Dubsky PC, Stift A et al (2008) Endorecta advancement flaps in the treatment of high anal fistula of cryptoglandular origin: full-thickness vs. mucosal-rectum flaps. Dis Colon Rectum 51(6):852-857

108. Dudukgian $\mathrm{H}, \mathrm{Abcarian} \mathrm{H}$ (2011) Why do we have so much trouble treating anal fistula? World J Gastroenterol 17(28):3292-3296

109. Durgun V, Perek A et al (2002) Partial fistulotomy and modified cutting seton procedure in the treatment of high extrasphincteric perianal fistulae. Dig Surg 19(1):56-58

110. Dutta G, Bain J et al (2015) Comparing Ksharasutra (Ayurvedic Seton) and open fistulotomy in the management of fistula-in-ano. J Nat Sci Biol Med 6(2):406-410

111. Dziki A, Bartos M (1998) Seton treatment of anal fistula: experience with a new modification. Eur J Surg 164(7):543-548

112. Dziki L, Mik M et al (2015) Treatment of perianal fistulas in Poland. Pol Przegl Chir 87(12):614-619

113. Echenique I, Mella JR et al (2008) Puerto Rico experience with plugs in the treatment of anal fistulas. Bol Asoc MedPR 100(1):8-12

114. Ege B, Leventoglu $S$ et al (2014) Hybrid seton for the treatment of high anal fistulas: results of 128 consecutive patients. Tech Coloproctol 18(2):187-193

115. Eisenhammer $S$ (1956) The internal anal sphincter and the anorectal abscess. Surg Gynecol Obstet 12:501-506

116. Eitan A, Koliada M et al (2009) The use of the loose seton technique as a definitive treatment for recurrent and persistent high trans-sphincteric anal fistulas: a long-term outcome. J Gastrointest Surg 13(6):1116-1119

117. Ellis CN (2007) Bioprosthetic plugs for complex anal fistulas: an early experience. J Surg Educ 64(1):36-40

118. Ellis CN (2010) Outcomes with the use of bioprosthetic grafts to reinforce the ligation of the intersphincteric fistula tract (bioLIFT procedure) for the management of complex anal fistulas. Dis Colon Rectum 53(10):1361-1364

119. Ellis CN (2010) Sphincter-preserving fistula management: what patients want. Dis Colon Rectum 53(12):1652-1655

120. Ellis CN, Clark S (2006) Fibrin glue as an adjunct to flap repair of anal fistulas: a randomized, controlled study. Dis Colon Rectum 49(11):1736-1740

121. Ellis CN, ClarkS (2007) Effect of tobacco smoking on advancement flap repair of complex anal fistulas. Dis Colon Rectum 50(4):459-463

122. Ellis CN, Rostas JW et al (2010) Long-term outcomes with the use of bioprosthetic plugs for the management of complex anal fistulas. Dis Colon Rectum 53(5):798-802

123. El-Tawil A (2011) Management of fistula-in-ano:an introduction. World J Gastroenterol 17(28):3271

124. Elting AW (1912) X. The treatment of fistula in ano: with especial reference to the whitehead operation. Ann Surg 56(5):744-752

125. Erhan Y, Sakarya A et al (2003) A case of large mucinous adenocarcinoma arising in a longstanding fistula-in-ano. Dig Surg 20(1):69-71

126. Fasth SB, Nordgren S et al (1990) Clinical course and management of suprasphincteric and extrasphincteric fistula-in-ano. Acta Chir Scand 156(5):397-402

127. Filingeri V, Gravante G et al (2004) Radiofrequency fistulectomy vs. diathermic fistulotomy for 
submucosal fistulas: a randomized trial. Eur Rev MedPharmacol Sci 8(3):111-116

128. Fisher OM, Raptis D et al (2014) An outcome and cost analysis of anal fistula plug insertion vs endorectal advancement flap for complex anal fistula. Colorectal Dis 17:619-626

129. Gaertner WB, Hagerman GF et al (2008) Fistulaassociated anal adenocarcinoma: good results with aggressive therapy. Dis Colon Rectum 51(7):1061-1067

130. Galis-Rozen E, Tulchinsky H et al (2010) Long-term outcome of loose seton for complex anal fistula: a two-centre study of patients with and without Crohn's disease. Colorectal Dis 12(4):358-362

131. Garces-Albir M, Garcia-Botello SA et al (2012) Quantifying the extent of fistulotomy. How much sphincter can we safely divide? A three-dimensional endosonographic study. Int J Colorectal Dis 27(8):1109-1116

132. García Olmo D, Vazquez Aragon P et al (1994) Multiple setons in the treatment of high perianal fistula. Br J Surg 81(1):136-137

133. Garcia-Aguilar J, Belmonte C et al (1998) Cutting seton versus two-stage seton fistulotomy in the surgical management of high anal fistula. Br J Surg 85(2):243-245

134. Garcia-AguilarJ,BelmonteCetal (1996) Analfistula surgery. Factors associated with recurrence and incontinence. Dis Colon Rectum 39(7):723-729

135. Garcia-Aguilar J, Davey CS et al (2000) Patient satisfaction after surgical treatment for fistula-inano. Dis Colon Rectum 43(9):1206-1212

136. Garcia-Olmo D, Herreros D et al (2009) Expanded adipose-derived stem cells for the treatment of complex perianal fistula: a phase II clinical trial. Dis Colon Rectum 52(1):79-86

137. Garg G, Tripathi M et al (2010) Demonstration of a tubercular fistula-in-ano on F-18 FDG PET/CT. Clin Nucl Med 35(4):300-302

138. Garg P (2009) To determine the efficacy of anal fistula plug in the treatment of high fistula-in-ano: an initial experience. Colorectal Dis 11(6):588-591

139. Garg P, Song J et al (2010) The efficacy of anal fistula plug in fistula-in-ano: a systematic review. Colorectal Dis 12(10):965-970

140. Gautier M, Godeberge P et al (2015) Easy clip to treat anal fistula tracts: a word of caution. Int J Colorectal Dis 30(5):621-624

141. Gemsenjager E (1996) Results with a new therapy concept in anal fistula: suture of the anal sphincter. Schweiz Med Wochenschr 126(47):2021-2025

142. Giamundo P, Esercizio L et al (2015) Fistulatract Laser Closure (FiLaC): long-term results and new operative strategies. Tech Coloproctol 19(8):449-453

143. Giamundo P, Geraci M et al (2013) Closure of fistula-in-ano with laser - FiLaC: an effective nove sphincter-saving procedure for complex disease. Colorectal Dis 16(2):110-115

144. Giordano P, Sileri P et al (2016) A prospective, multicentre observational study of Permacol collagen paste for anorectal fistula: preliminary results. Colorectal Dis 18(3):286-294. doi:10.1111/ codi.13112

145. Gisbertz SS, Sosef MN et al (2005) Treatment of fistulas in ano with fibrin glue. Dig Surg 22(1-2):91-94

146. Goertz O, Kapalschinski N et al (2012) Wound healing complications in smokers, non-smokers and after abstinence from smoking. Chirurg 83(7):652-656

147. Golub RW, Wise WE Jr. et al (1997) Endorectal mucosal advancement flap: the preferred method for complex cryptoglandular fistula-in-ano. J Gastrointest Surg 1(5):487-491

148. Gonzalez-Ruiz C, Kaiser AM et al (2006) Intraoperative physical diagnosis in the management of anal fistula. Am Surg 72(1):11-15

149. Goos M, Manegold P et al (2015) Long-term results after endoanal advancement flap repair for fistulas-in-ano. How important is the aetiology? Int JColorectal Dis 30(3):413-419

150. Göttgens KW, Janssen PT et al (2015) Longterm outcome of low perianal fistulas treated by fistulotomy: a multicenter study. Int J Colorectal Dis 30(2):213-219

151. Göttgens KW, Smeets RR et al (2015) Systematic review and meta-analysis of surgical interventions for high cryptoglandular perianal fistula. Int J Colorectal Dis 30(5):583-593

152. Göttgens KW, Smeets RR et al (2016) Operative Verfahren bei hohen kryptoglandulären Analfisteln: Systematische Übersicht und Metaanalyse. Coloproctology 38(2):93-105. doi:10.1007/s00053015-0069-9

153. Göttgens KW, Vening W et al (2014) Long-term results of mucosal advancement flap combined with platelet-rich plasma for high cryptoglandular perianal fistulas. Dis Colon Rectum 57(2):223-227

154. Graf W, Pahlman L et al (1995) Functional results after seton treatment of high transsphincteric anal fistulas. Eur J Surg 161(4):289-291

155. Gravante G, Giordano P (2008) The role of threedimensional endoluminal ultrasound imaging in the evaluation of anorectal diseases: a review. Surg Endosc 22(7):1570-1578

156. Greenberg R, Werbin N et al (2002) Repair of anorectal fistulas using fibrin glue tissue adhesive - preliminary experience in 15 patients. Harefuah 141(12):1021-1024, 1091

157. Guadalajara H, Herreros D et al (2012) Long-term follow-up of patients undergoing adipose-derived adult stem cell administration to treat complex perianal fistulas. Int J Colorectal Dis 27(5):595-600

158. Guenaga KF, Matos D et al (2003) Mechanical bowel preparation for elective colorectal surgery. Cochrane Database Syst Rev(2):CD001544. doi:10. 1002/14651858.cd001544

159. Gupta PJ (2003) Radiosurgical fistulotomy; an alternative to conventional procedure in fistula in ano. Curr Surg 60(5):524-528

160. GuptaPJ, Gupta SN etal (2012) Which treatment for anal fistula? Cut or cover, plug or paste, loop or lift ActaChir lugosl 59(2):15-20

161. Gürer A, Özlem N et al (2007) A novel material in seton treatment of fistula-in-ano. Am J Surg 193(6):794-796

162. Gustafsson UM, Graf W (2002) Excision of anal fistula with closure of the internal opening: functional and manometric results. Dis Colon Rectum 45(12):1672-1678

163. Gustafsson UM, GrafW (2006) Randomized clinical trial of local gentamicin-collagen treatment in advancement flap repair for anal fistula. Br J Surg 93(10):1202-1207

164. Gustafsson UM, Kahvecioglu B et al (2001) Endoanal ultrasound or magnetic resonance imaging for preoperative assessment of anal fistula: a comparative study. Colorectal Dis 3(3):189-197

165. Hall JF, Bordeianou L et al (2014) Outcomes after operations for anal fistula: results of a prospective multicenter, regional study. Dis Colon Rectum 57(11):1304-1308

166. Hämäläinen KP, Sainio AP (1997) Cutting seton for anal fistulas: high risk of minor control defects.
Dis Colon Rectum 40(12):1443-1446 (discussion 1447)

167. Hamel CT, Marti WR et al (2004) Simplified placement and management of cutting setons in the treatment of transsphincteric anal fistula: technical note. Int J Colorectal Dis 19(4):354-356 (discussion 357-8)

168. Hammond TM, Grahn MF et al (2004) Fibrin glue in the management of anal fistulae. Colorectal Dis 6(5):308-319

169. Hammond TM, Knowles CH et al (2006) The Snug Seton: short and medium term results of slow fistulotomy for idiopathic anal fistulae. Colorectal Dis 8(4):328-337

170. HammondTM, Porrett TRetal (2010) Management of idiopathic anal fistula using cross-linked collagen: a prospective phase 1 study. Colorectal Dis 13:94-104

171. Han JG, Wang ZJ et al (2011) Long-term outcomes of human acellular dermal matrix plug in closure of complex anal fistulas with a single tract. Dis Colon Rectum 54(11):1412-1418

172. Hanley PH (1978) Rubber band seton in the management of abscess-anal fistula. Ann Surg 187(4):435-437

173. Hasse C, Brune $M$ et al (2004) Laterale, partielle Sphinkteromyotomie zur Therapie der chronischen Analfissur - Langzeitergebnisse einer epidemiologischen Kohortenstudie. Chirurg 75(2):160-167

174. Held D, Khubchandani l et al (1986) Management of anorectal horseshoe abscess and fistula. Dis Colon Rectum 29(12):793-797

175. Herman G, Desfosses L (1880) Sur la musquese de la region cloacale du rectum. C R Acad Sci 90:1301-1302

176. Herreros MD, Garcia-Arranz M et al (2012) Autologous expanded adipose-derived stem cells for the treatment of complex cryptoglandular perianal fistulas: a phase III randomized clinical trial (FATT 1: fistula Advanced Therapy Trial 1) and longterm evaluation. Dis Colon Rectum 55(7):762-772

177. Heydari A, Attina GM et al (2013) Bioabsorbable synthetic plug in the treatment of anal fistulas. Dis Colon Rectum 56(6):774-779

178. Himpson RC, Cohen CR et al (2009) An experimentally successful new sphincter-conserving treatment for anal fistula. Dis Colon Rectum 52(4):602-608

179. Hirschburger M, Schwandner T et al (2014) Fistulectomy with primary sphincter reconstruction in the treatment of high transsphincteric anal fistulas. Int JColorectal Dis 29(2):247-252

180. Hjortrup A, Moesgaard F et al (1991) Fibrin adhesive in the treatment of perineal fistulas. Dis Colon Rectum 34(9):752-754

181. Ho KS, Ho YH (2005) Controlled, randomized trial of island flap anoplasty for treatment of trans-sphincteric fistula-in-ano: early results. Tech Coloproctol 9(2):166-168

182. HoKS, Tsang Cetal (2001) Prospective randomised trial comparing ayurvedic cutting seton and fistulotomy for low fistula-in-ano. Tech Coloprocto 5(3):137-141

183. Ho YH, Tan Met al (1998) Marsupialization of fistulotomy wounds improves healing: a randomized controlled trial. Br JSurg 85(1):105-107

184. Ho YH, Tsang C et al (2000) Anal sphincter injuries from stapling instruments introduced transanally: randomized, controlled study with endoanal ultrasound and anorectal manometry. Dis Colon Rectum 43(2):169-173 
185. Holzheimer RG, Siebeck M (2006) Treatment procedures for anal fistulous cryptoglandular absces s - how to get the best results. Eur J Med Res 11(12):501-515

186. Hong KD, Kang S et al (2014) Ligation of intersphincteric fistula tract (LIFT) to treat anal fistula: systematic review and meta-analysis. Tech Coloproctol 18(8):685-691

187. Hongo Y, Kurokawa A et al (1997) Open coring-out (function-preserving) technique for low fistulas. Dis Colon Rectum 40(10Suppl):S104-S106

188. Hori M, Oto A et al (2009) Diffusion-weighted MRI: a new tool for the diagnosis of fistula in ano. J Magn Reson Imaging 30(5):1021-1026

189. Hossack T, Solomon MJ et al (2005) Anocutaneous flap repair for complex and recurrent supra-sphincteric anal fistula. Colorectal Dis 7(2):187-192

190. Howard D, DeLancey JO et al (1999) Fistula-inano after episiotomy. Obstet Gynecol 93(5 Pt 2):800-802

191. http://www.cebm.net/

192. Hübner N-O, Assadian $O$ et al (2007) Anforderungen an die Wundreinigung mit Wasser. GMS Krankenhhyg Interdiszip 2(2):1-4

193. Isbister WH, Al Sanea N (2001) The cutting seton: an experience at King Faisal Specialist Hospital. Dis Colon Rectum 44(5):722-727

194. Iwadare J, Sumikoshi Y et al (1997) Muscle-filling procedure for transsphincteric fistulas. Dis Colon Rectum 40(10Suppl):S102-S103

195. Jacob TJ, Keighley MR et al (2010) Surgical intervention for chronic anorectal fistula (Review). Cochrane Database Syst Rev:CD006319. doi:14651858.CD006319.pub2

196. Jain BK, Vaibhaw K et al (2011) Comparison of a fistulectomy and a fistulotomy with marsupialization in the management of a simple anal fistula: a randomized, controlled pilot trial. J Korean Soc Coloproctol 28(2):78-82

197. Jain SK, Kaza RCM et al (2008) Role of cyanoacrylate in the management of low fistula in ano: a prospective study. Int J Colorectal Dis 23:255-358

198. Jarrar A, Church J (2012) Advancement flap repair: a good option for complex anorectal fistulas. Dis Colon Rectum 54(12):1537-1541

199. Johnson EK, Gaw JU et al (2006) Efficacy of anal fistula plug vs. fibrin glue in closure of anorectal fistulas. Dis Colon Rectum 49(3):371-376

200. Joos AK, Bussen D et al (2009) Abszess, Analfistel, Analfissur.AllgViszeralchirUp2date3(04):221-236

201. Joos AK, Palma P et al (2008) Enteral vs parenteral nutrition in reconstructive anal surgery - a prospective-randomized trial. Colorectal Dis 10(6):605-609

202. Jordan J, Roig JV et al (2010) Risk factors for recurrence and incontinence after anal fistula surgery. Colorectal Dis 12(3):254-260

203. Joy HA, Williams JG (2002) The outcome of surgery for complex anal fistula. Colorectal Dis 4(4):254-261

204. Jun SH, Choi GS (1999) Anocutaneous advancement flap closure of high anal fistulas. Br J Surg 86(4):490-492

205. Jurczak F, Laridon JY et al (2004) Biological fibrin used in anal fistulas: 31 patients. Ann Chir 129(5):286-289

206. Kelly ME, Heneghan HM et al (2014) The role of loose seton in the management of anal fistula: a multicenter study of 200 patients. Tech Coloproctol 18(10):915-919

207. Kennedy HL, Zegarra JP (1990) Fistulotomy without external sphincter division for high anal fistulae. Br J Surg 77(8):898-901
208. Khafagy W, Omar W et al (2010) Treatment of anal fistulas by partial rectal wall advancement flap or mucosal advancement flap: a prospective randomized study. Int J Surg 8(4):321-325

209. Kim Y, Park YJ (2009) Three-dimensional endoanal ultrasonographic assessment of an anal fistula with and without $\mathrm{H}(2) \mathrm{O}(2)$ enhancement. World J Gastroenterol 15(38):4810-4815

210. KleifJ, Hagen Ketal (2011) Acceptable resultsusing plug for the treatment of complexanal fistulas. Dan Med Bull 58(3):A4254

211. Kleinübing $\mathrm{H}$ Jr., Jannini JF et al (2007) The role of transperineal ultrasonography in the assessment of the internal opening of cryptogenic anal fistula. Tech Coloproctol 11(4):327-331

212. Kochhar G, Saha S et al (2014) Video-assisted anal fistula treatment. JSLS 18(3):e2014.00127

213. Köckerling F, von Rosen Tetal (2014) Modified plug repair with limited sphincter sparing fistulectomy in the treatment of complex anal fistulas. Front Surg 1:17

214. Köckerling F, Alam NN, Narang SK, Daniels IR et al (2015) Treatment of fistula-in-ano with fistula plug - a review under special consideration of the technique. Front Surg 2:55

215. Kodner IJ, Mazor A et al (1993) Endorectal advancement flap repair of rectovaginal and other complicated anorectal fistulas. Surgery 114(4):682-689 (discussion 689-690)

216. Köhler A, Athanasiadis S (1996) Die anodermale Verschiebelappenplastik als alternative Behandlungsmethode zu den endorectalen Verschlußtechniken bei der Therapie hoher Analfisteln. Eine prospektive Studie bei 31 Patienten. Chirurg 67(12):1244-1250

217. Köhler A, Athanasiadis S et al (1997) Die Analfiste - Ein Plädoyer für die kontinente Fistulektomie. Coloproctology 19(5):186-203

218. Köhler A, Athanasiadis S et al (1999) Vorgehen be primär nicht klassifizierbaren Analfisteln. Chirurg 70(5):578-581

219. Köhler A, Risse-Schaaf A et al (2004) Treatment for horseshoe fistulas-in-ano with primary closure of the internal fistula opening: a clinical and manometric study. Dis Colon Rectum 47(11):1874-1882

220. Kraemer M, Picke D (2011) Fistelspaltung und primäre Sphinkterrekonstruktion zur Behandlung von Analfisteln. Coloproctology 33(2):104-108

221. Kucharczyk A, Kolodziejczak M et al (2014) Autologous growth factors used for the treatment of recurrent fistula-in-ano preliminary results. Tech Coloproctol 18(3):317-318

222. Kügler S (1966) Die Kontinenz nach Spaltung anorectaler Fisteln. Chirurg 37(2):64-66

223. Kuijpers HC, Schulpen T (1985) Fistulography for fistula-in-ano. Is it useful? Dis Colon Rectum 28(2):103-104

224. Kuypers HC(1984) Use of the seton in the treatment of extrasphincteric anal fistula. Dis Colon Rectum 27(2):109-110

225. Law PJ, Talbot RW et al (1989) Anal endosonography in the evaluation of perianal sepsis and fistula in ano. Br J Surg 76(7):752-755

226. Lawes DA, Efron JE et al (2008) Early experience with the bioabsorbable anal fistula plug. World J Surg 32(6):1157-1159

227. Lechner P (1991) Der Mucosa-Verschiebelappen in der Behandlung supra- und hoch-transsphincterer Analfisteln. Chirurg 62(12):891-894

228. Lee $\mathrm{CL}$, Lu J et al (2015) Long-term outcome following advancement flaps for high anal fistulas in an Asian population: a single institution's experience. Int J Colorectal Dis 30(3):409-412
229. LeFevre C, Erekson EA et al (2010) Fistula-in-ano: an uncommon cause of chronic vulvar symptoms. Obstet Gynecol 115(2 Pt 2):421-423

230. Lehmann JP, Graf W (2013) Efficacy of LIFT for recurrent anal fistula. Colorectal Dis 15(5):592-595

231. Lengyel AJ, Hurst NG et al (2002) Pre-operative assessment of anal fistulas using endoanal ultrasound. Colorectal Dis 4(6):436-440

232. Lenisa L, Espin-Basany E et al (2010) Anal fistula plug is a valid alternative option for the treatment of complex anal fistula in the long term. Int J Colorectal Dis 25(12):1487-1493

233. Lentner A, Wienert V (1996) Long-term, indwelling setons for low transsphincteric and intersphincteric anal fistulas. Experience with 108 cases. Dis Colon Rectum 39(10):1097-1101

234. Lewis R, Lunniss PJ et al (2012) Novel biologica strategies in the management of anal fistula. Colorectal Dis 14(12):1445-1455

235. Lilius HG (1968) Fistula-in-ano, an investigation of human foetal anal ducts and intramuscular glands and a clinical study of 150 patients. Acta Chir Scand Suppl 383:7-88

236. Limura $E$, Giordano $P$ (2015) Modern management of anal fistula. World J Gastroenterol 21(1):12-20

237. Lindsey I, Humphreys MM et al (2002) The role of anal ultrasound in the management of anal fistulas. Colorectal Dis 4(2):118-122

238. Lindsey I, Smilgin-Humphreys MM et al (2002) A randomized, controlled trial of fibrin glue vs. conventional treatment for anal fistula. Dis Colon Rectum 45(12):1608-1615

239. Liu WY, Aboulian A et al (2013) Long-term results of ligation of intersphincteric fistula tract (LIFT) for fistula-in-ano. Dis Colon Rectum 56(3):343-347

240. Lo OSH, Wei R et al (2012) Ligation of intersphincteric fistula tract procedure for the management of cryptoglandular anal fistulas. Surg Pract 16:120-121

241. Losonc C, Podlinski C et al (2012) Nicht heilende Analfistel - Frühzeitige Indikation zur Schnittbilddiagnostik. Chir Prax 74:305-308

242. Lubowski DZ (2010) Surgical treatment of anal fistula: a track not conquered. ANZ J Surg 80(5):301-303

243. Lunniss PJ, Faris B et al (1993) Histological and microbiological assessment of the role of microorganisms in chronic anal fistula. Br J Surg 80(8):1072

244. Lunniss PJ, Kamm MA et al (1994) Factors affecting continence after surgery for anal fistula. Br J Surg 81(9):1382-1385

245. Lunniss PJ, Phillips RK (1994) Surgical assessment of acute anorectal sepsis is a better predictor of fistula than microbiological analysis. Br J Surg 81(3):368-369

246. Lupinacci RM, Vallet C et al (2010) Treatment of fistula-in-ano with the Surgisis((R)) AFP(TM) anal fistula plug. GastroenterolClin Biol34(10):549-553

247. Madbouly KM, El Shazly W et al (2014) Ligation of intersphincteric fistula tract versus mucosal advancement flap in patients with high transsphincteric fistula-in-ano: a prospective randomized trial. Dis Colon Rectum 57(10):1202-1208

248. Malik Al, Nelson RL (2008) Surgical management of anal fistulae: a systematic review. Colorectal Dis 10(5):420-430

249. Malouf AJ, Buchanan GN et al (2002) A prospective audit of fistula-in-ano at St. Mark's hospital. Colorectal Dis 4(1):13-19

250. Maor Y, Chowers Y et al (2005) Endosonographic evaluation of perianal fistulas and abscesses: comparison of two instruments and assessment 
of the role of hydrogen peroxide injection. J Clin Ultrasound 33(5):226-232

251. Maralcan G, Baskonus I et al (2006) The use of fibrin glue in the treatment of fistula-in-ano: a prospective study. Surg Today 36(2):166-170

252. Maralcan G, Baskonus I et al (2011) Long-term results in the treatment of fistula-in-ano with fibrin glue: a prospective study. J Korean Surg Soc 81(3):169-175

253. Marks CG, Ritchie JK (1977) Anal fistulas at St Mark's Hospital. Br JSurg 64(2):84-91

254. Marti L, Nussbaumer P et al (2001) Das perianale mucinöse Adenokarzinom. Ein weiterer Grund für die histologische Untersuchung bei Analfistel oder Anorektalabszess. Chirurg 72(5):573-577

255. Matos D, Lunniss PJ et al (1993) Total sphincter conservation in high fistula in ano: results of a new approach. Br JSurg 80(6):802-804

256. McCourtney JS, Finlay IG (1995) Setons in the surgical management of fistula in ano. Br J Surg 82(4):448-452

257. McCourtney JS, Finlay IG (1996) Cutting seton without preliminary internal sphincterotomy in management of complex high fistula-in-ano. Dis Colon Rectum 39(1):55-58

258. McElwain JW, MacLean MD et al (1975) Anorectal problems: experience with primary fistulectomy for anorectal abscess, a report of 1,000 cases. Dis Colon Rectum 18(8):646-649

259. McGee MF, Champagne BJ et al (2010) Tract length predicts successful closure with anal fistula plug in cryptoglandular fistulas. Dis Colon Rectum 53(8):1116-1120

260. Meinero P, Mori L (2011) Video-assisted anal fistula treatment (VAAFT): a novel sphincter-saving procedure for treating complex anal fistulas. Tech Coloproctol 15(4):417-422

261. Meinero P, Mori L (2012) Video-assisted anal fistula treatment (VAAFT): a novel sphincter-saving procedure to repair complex anal fistulas. Tech Coloproctol 16(6):469-470

262. Meinero P, Mori L et al (2014) Video-assisted anal fistula treatment: a new concept of treating anal fistulas. Dis Colon Rectum 57(3):354-359

263. Memon AA, Murtaza G et al (2011) Treatment of complex fistula in ano with cable-tie seton: a prospective case series. ISRN Surg 2011:636952 doi:10.5402/2011/636952

264. Mennigen R, Laukotter Met al (2015) The OTSC((R)) proctology clip system for the closure of refractory anal fistulas. Tech Coloproctol 19(4):241-246

265. Mentes BB, Tezcaner T et al (2006) Results of lateral internal sphincterotomy for chronic anal fissure with particular reference to quality of life. Dis Colon Rectum 49(7):1045-1051

266. Miller GV, Finan PJ (1998) Flap advancement and core fistulectomy for complex rectal fistula. $\mathrm{Br}$ J Surg 85(1):108-110

267. Misra MC, Kapur BM (1988) A new non-operative approach to fistula in ano. Br J Surg 75(11):1093-1094

268. Mitalas LE, Dwarkasing RS et al (2011) Is the outcome of transanal advancement flap repair affected by the complexity of high transsphincteric fistulas? Dis Colon Rectum 54(7):857-862

269. Mitalas LE, Gosselink MP et al (2009) Adverse effect of BioGlue on the outcome of transana advancement flap repair. Dis Colon Rectum 52(4):754 (author reply 755)

270. Mitalas LE, Gosselink MP et al (2009) Required length of follow-up after transanal advancement flap repair of high transsphincteric fistulas. Colorectal Dis 11(7):726-728
271. Mitalas LE, Gosselink MP et al (2007) Repeat transanal advancement flap repair: impact on the overall healing rate of high transsphincteric fistulas and on fecal continence. Dis Colon Rectum 50(10):1508-1511

272. Mitalas LE, van Onkelen RS et al (2012) Identification of epithelialization in high transsphincteric fistulas. Tech Coloproctol 16:113-117

273. Mitalas LE, van Wijk JJ et al (2010) Seton drainage prior to transanal advancement flap repair: usefu or not? Int J Colorectal Dis 25(12):1499-1502

274. Mohite JD, Gawai RS et al (1997) Ksharsootra (medicated seton) treatment for fistula-in-ano. Indian J Gastroenterol 16(3):96-97

275. Moscowitz I, Baig MK et al (2003) Accuracy of hydrogen peroxide enhanced endoanal ultrasonography in assessment of the internal opening of an anal fistula complex. Tech Coloproctol 7(3):133-137

276. Muhlmann MD, Hayes JL et al (2011) Complex ana fistulas: plug or flap? ANZJ Surg 81(10):720-724

277. Murad-Regadas SM, Regadas FS et al (2010) The role of 3-dimensional anorectal ultrasonography in the assessment of anterior transsphincteric fistula. Dis Colon Rectum 53(7):1035-1040

278. Mushaya C, Bartlett L et al (2012) Ligation of intersphincteric fistula tract compared with advancement flap for complex anorectal fistulas requiring initial seton drainage. Am J Surg 204(3):283-289

279. Mylonakis E, Katsios C et al (2001) Quality of life of patients after surgical treatment of anal fistula; the role of anal manometry. Colorectal Dis 3(6):417-421

280. Nagendranath C, Saravanan MN et al (2014) Peroxide-enhanced endoanal ultrasound in preoperative assessment of complex fistula-inano. Tech Coloproctol 18(5):433-438

281. Narang SK, Jones C et al (2015) Delayed absorbable synthetic plug (GORE(R) BIO-A(R)) for the treatment of fistula-in-ano: a systematic review. Colorectal Dis 18(1):37-44

282. Navarro-Luna A, Garcia-Domingo Ml et al (2004) Ultrasound study of anal fistulas with hydrogen peroxide enhancement. Dis Colon Rectum 47(1):108-114

283. Nessim A, Wexner SD et al (1999) Is bowel confinement necessary after anorectal reconstructive surgery? A prospective, randomized, surgeonblinded trial. Dis Colon Rectum 42(1):16-23

284. Niyogi A, Agarwal T et al (2010) Management of perianal abscess and fistula-in-ano in children. Eu JPediatr Surg 20(1):35-39

285. Noble GH (1902) A new operation for complex laceration of the perineum designed for the purpose of eliminating danger of infection from the rectum. Trans Am Gynecol Soc 27:357-363

286. Nwaejike N, Gilliland R(2007) Surgery for fistula-inano: an audit of practise of colorectal and general surgeons. Colorectal Dis 9(8):749-753

287. Oh C (1983) Management of high recurrent anal fistula. Surgery 93(2):330-332

288. Ommer A, Athanasiadis S et al (1999) Die chirurgische Behandlung des anorektalen Abszes ses. Sinn und Unsinn der primären Fistelsuche. Coloproctology 21(5):161-169

289. Ommer A, Athanasiadis S et al (2000) Die Bedeutung derStomaanlageim Rahmen der Behandlung der komplizierten Analfisteln und der rektovaginalen Fisteln. Coloproctology 22(1):14-22

290. Ommer A, Herold A et al (2011) S3-Leitlinie Kryptoglanduläre Analfistel. Coloproctology 33(5):295-324
291. Ommer A, Herold A et al (2012) Gore BioA Fistula Plug in the treatment of high anal fistulas - initial results from a German multicenter-study. Ger Med Sci 10:Doc13

292. Ommer A, Herold A et al (2012) Analfistelplug. Coloproctology 34:341-351

293. Ommer A, Sailer M (2011) Therapieoptionen bei Komplikationen nach Analfisteloperationen. Chir Prax 73(4):657-668

294. Ommer A, Schmidt C et al (2012) Gore BioA Fistelplug zur Behandlung hoher Analfisteln. Coloproctology 34(1):24

295. Ooi K, Skinner l et al (2011) Managing fistula-inano with ligation of the intersphincteric fistula tract procedure: the Western Hospital experience. Colorectal Dis 14(5):599-603

296. O'Riordain DS, Datta I et al (2012) A systematic review of the anal fistula plug for patients with Crohn's and Non-Crohn's related fistula-in-ano. Dis Colon Rectum 55(3):351-358

297. Ortiz H, Marzo J (2000) Endorectal flap advancement repair and fistulectomy for high transsphincteric and suprasphincteric fistulas. Br J Surg 87(12):1680-1683

298. Ortiz H, Marzo J et al (2009) Randomized clinical trial of anal fistula plug versus endorectal advancement flap for the treatment of high cryptoglandular fistula in ano. Br J Surg 96(6):608-612

299. Ortiz H, Marzo M et al (2008) Length of follow-up after fistulotomy and fistulectomy associated with endorectal advancement flap repair for fistula in ano. Br JSurg 95(4):484-487

300. Owen G, Keshava A et al (2010) Plugs unplugged. Anal fistula plug: the Concord experience. ANZ J Surg 80(5):341-343

301. Öztürk E (2015) Treatment of recurrent anal fistula using an autologous cartilage plug: a pilot study. Tech Coloproctol 19(5):301-307

302. Öztürk E, Gülcü B (2014) Laser ablation of fistula tract: a sphincter-preserving method for treating fistula-in-ano. Dis Colon Rectum 57(3):360-364

303. Ozuner G, Hull TL et al (1996) Long-term analysis of the use of transanal rectal advancement flaps for complicated anorectal/vaginal fistulas. Dis Colon Rectum 39(1):10-14

304. Pagano G, Biondo G et al (2004) Complex anal fistula surgery: personal experience. Chir Ital 56(4):523-527

305. Paksoy M, Ozben V et al (2010) An atypical etiology of suprasphincteric fistula: a forgotten surgical material. Case Rep Med 2010:189846. doi:10.1155/ 2010/189846

306. Panidis S, Papadopoulos VN et al (2010) The use of fibrin glue in the treatment of complex perianal fistulae: a case report. Tech Coloproctol 14(S1):19-20

307. Papapolychroniadis C, Kaimakis D et al (2004) A case of mucinous adenocarcinoma arising in longstanding multiple perianal and presacral fistulas. Tech Coloproctol 8(Suppl 1):s138-s140

308. PapavramidisTS, Pliakos letal (2010) Management of an extrasphincteric fistula in an HIV-positive patient by using fibrin glue: a case report with tips and tricks. BMC Gastroenterol 10:18

309. Parés D (2011) Pathogenesis and treatment of fistula in ano. Br JSurg 98(1):2-3

310. Park JJ, Cintron JR et al (2000) Repair of chronic anorectal fistulae using commercial fibrin sealant. Arch Surg 135(2):166-169

311. Parkash S, Lakshmiratan V et al (1985) Fistula-inano: treatment by fistulectomy, primary closure and reconstitution. Aust NZJ Surg 55(1):23-27

312. Parks AG, Gordon PH (1976) Fistula-in-Ano: perineal fistula of intra-abdominal or intrapelvic 
origin simulating fistula-in-ano - report of seven cases. Dis Colon Rectum 19(6):500-506

313. Parks AG, Gordon PH et al (1976) A classification of fistula-in-ano. BrJSurg 63(1):1-12

314. Parks AG, Stitz RW (1976) The treatment of high fistula-in-ano. Dis Colon Rectum 19(6):487-499

315. Patrlj L, Kocman B et al (2000) Fibrin glueantibiotic mixture in the treatment of anal fistulae: experience with 69 cases. Dig Surg 17(1):77-80

316. Patton V, Chen CM et al (2015) Long-term results of the cutting seton for high anal fistula. ANZ J Surg 85(10):720-727

317. Pearl RK, Andrews JR et al (1993) Role of the seton in the management of anorectal fistulas. Dis Colon Rectum 36(6):573-577 (discussion 577-579)

318. Perez F, Arroyo A et al (2006) Stapled endorectal mucosectomy for high extrasphincteric fistulain-ano: preliminary report. Dis Colon Rectum 49(4):519-523 (PPH-01-Stapler (Ethicon Endosurgery))

319. Perez F, Arroyo A et al (2005) Fistulotomy with primary sphincter reconstruction in the management of complex fistula-in-ano: prospective study of clinical and manometric results. J Am Coll Surg 200(6):897-903

320. Perez F, Arroyo A et al (2006) Randomized clinical and manometric study of advancement flap versus fistulotomy with sphincter reconstruction in the management of complex fistula-in-ano. Am J Surg 192(1):34-40

321. Pescatori M, Ayabaca S et al (2004) Can anal manometry predict anal incontinence after fistulectomy in males? Colorectal Dis 6(2):97-102

322. Pescatori M, Ayabaca SM et al (2006) Marsupialization of fistulotomy and fistulectomy wounds improves healing and decreases bleeding: a randomized controlled trial. Colorectal Dis 8(1):11-14

323. Phillips B, Ball C et al (2009) Oxford Centre for Evidence-based medicine - levels of evidence. http://www.cebm.net/oxford-centre-evidencebased-medicine-levels-evidence-march-2009/

324. Pikarsky AJ, Nogueras JJ et al (2000) Surgical workshop: a new modification with the use of a seton in treating anal fistula. Tech Coloproctol 4:109-110

325. Pinedo MG, Caselli MG (2010) Modified looseseton technique for the treatment of complex ana fistulas. Colorectal Dis 12:e310-e313

326. Pinsk I, Seppala R et al (2010) Anography: a technique for determining the location of the internal opening in perianal fistula. Colorectal Dis 12(9):896-900

327. PomerriF, DodiGetal (2010) Anal endosonography and fistulography for fistula-in-ano. Radiol Med 115(5):771-783

328. Poon CM, Ng DC et al (2008) Recurrence pattern of fistula-in-ano in a Chinese population. J Gastrointestin Liver Dis 17(1):53-57

329. Prosst RL, Ehni W (2012) The OTSC(R) Proctology clip system for anorectal fistula closure: the 'anal fistula claw': case report. Minim Invasive Ther Allied Technol 21(4):307-312

330. Prosst RL, Ehni $W$ et al (2013) The OTSC(R) Proctology clip system for anal fistula closure: first prospective clinical data. Minim Invasive The Allied Technol 22(5):255-259

331. Prosst RL, Herold A et al (2011) The anal fistula claw: the OTSC clip for anal fistula closure. Colorectal Dis 14(9):1112-1117

332. Pu YW, Xing CG et al (2012) Fistula plug versus conventional surgical treatment for anal fistulas. A system review and meta-analysis. Saudi Med J 33(9):962-966
333. Queralto M, Portier Get al (2010) Efficacy of synthetic glue treatment of high crypoglandular fistulain-ano. Gastroenterol Clin Biol 34(8-9):477-482

334. Rakinic J, Poola VP (2014) Hemorrhoids andfistulas: new solutions to old problems. Curr Probl Surg 51(3):98-137

335. Ramanujam PS, Prasad ML (1983) The role of seton in fistulotomy of the anus. Surg Gynecol Obstet 157(11):419-422

336. Ramesh PB (2013) Anal fistula with foot extensiontreated by kshara sutra (medicated seton) therapy: a rare case report. Int J Surg Case Rep 4(7):573-576

337. Ratto C, Grillo E et al (2005) Endoanal ultrasoundguided surgery for anal fistula. Endoscopy 37(8):722-728

338. Ratto C, Litta F et al (2015) Fistulotomy or fistulectomy and primary sphincteroplasty for anal fistula (FIPS): a systematic review. Tech Coloproctol 19(7):391-400

339. Ratto C, Litta F (2012) Gore Bio-A((R)) Fistula Plug, a New Sphincter-Sparing Procedure For Complex Anal Fistula. Colorectal Dis 14:e264-e269

340. Ratto C, Litta F et al (2013) Fistulotomy with endto-end primary sphincteroplasty for anal fistula: results from a prospective study. Dis Colon Rectum 56(2):226-233

341. Read DR, Abcarian H (1979) A prospective survey of 474 patients with anorectal abscess. Dis Colon Rectum 22(8):566-568

342. Reznick RK, Bailey HR (1988) Closure of the internal opening for treatment of complex fistula-in-ano. Dis Colon Rectum 31(2):116-118

343. Riedler L, Papp C et al (1978) Chirurgische Spätergebnisse bei 107 Patienten mit anorektalen Fisteln. Leber Magen Darm 8(1):55-58

344. Riss S, Bachleitner-Hofmann T et al (2014) The comfort drain: a new device for treating complex anal fistula. Tech Coloproctol 18(11):1133-1135

345. Ritchie RD, Sackier JM et al (2009) Incontinence rates after cutting seton treatment for anal fistula. Colorectal Dis 11(6):564-571

346. Rizzo JA, Naig AL (2010) Anorectal abscess and fistula-in-ano: evidence-based management. Surg Clin North Am 90(1):45-68 (Table of Contents)

347. Robert-Koch-Institut (2005) Empfehlung der Kommission für Krankenhaushygiene und Infektionsprävention (KRINKO) beim Robert KochInstitut (RKI) Infektionsprävention in Heimen. Bundesgesundheitsblatt 48:1061-1080

348. Roig JV, Garcia-Armengol J (2010) Fistulectomy and sphincteric reconstruction for complex cryptoglandular fistulas. Colorectal Dis 12(7 Online):e145-e152

349. Roig JV, Jordan J et al (2009) Changes in anorectal morphologic and functional parameters after fistula-in-ano surgery. Dis Colon Rectum 52(8):1462-1469

350. Rojanasakul A (2009) LIFT procedure: a simplified technique for fistula-in-ano. Tech Coloproctol 13(3):237-240

351. Rojanasakul A (2010) "Comments to the invited comment "LIFT procedure: a simplified technique for fistula in ano, by P. J. Lunniss..' Tech Coloprocto 14(1):53-54

352. Rojanasakul A, Pattanaarun J et al (2007) Total anal sphincter saving technique for fistula-in-ano; the ligation of intersphincteric fistula tract. J Med Assoc Thai 90(3):581-586

353. Rollinson PD, Dundas SA (1984) Adenocarcinoma of sigmoid colon seeding into pre-existing fistula in ano. Br J Surg 71(9):664-665

354. Rosen DR, Kaiser AM (2016) Definitive seton management for Transsphincteric fistula-in-ano: harm or charm? Colorectal Dis 18(5): 488-95
355. Safar B, Jobanputra S et al (2009) Anal fistula plug: initial experience and outcomes. Dis Colon Rectum 52(2):248-252

356. Sahni VA, Ahmad R et al (2008) Which method is best for imaging of perianal fistula? Abdom Imaging 33(1):26-30

357. Saigusa N, Fujisaki H et al (2015) "Staged seton fistulotomy after restorative proctocolectomy with ileal pouch-anal anastomosis accompanied by a decline in anal pressure during manometry... Int J Colorectal Dis 30(3):421-423

358. Sainio P (1984) “Fistula-in-ano in a defined population. Incidence and epidemiological aspects.,.. Ann Chir Gynaecol 73(4):219-224

359. Saino P, Husa A (1985) “A prospective manometric study of the effect of anal fistula surgery on anorectal function.". Acta Chir Scand 151:279-288

360. Salim AS, Ahmed TM (2001) KTP-Laser and fibrin glue for treatment of fistulae in ano. Saudi Med J 22(11):1022-1024

361. Sangwan YP, Rosen L et al (1994) Is simple fistulain-ano simple? Dis Colon Rectum 37(9):885-889

362. Santoro GA, Fortling B (2006) New technical developments in endoanal and endorectal ultrasonography. In: Santoro GA, Di Falco G (Hrsg) Benign anorectal diseases. Diagnosis with endoanal and endorectal ultrasonography and new treatment options. Springer, Milan, S13-26

363. Bharathi SR, Sharma V et al (2010) Evidence based switch to perianal blockfor ano-rectal surgeries. In JSurg 8(1):29-31

364. Sarici IS, Sevim Y et al (2016) A new method for evaluation of perianal fistula: digital infrared thermal imaging. Tech Coloproctol 20(3):193-194

365. Schaefer O, Lohrmann Cet al (2004) Assessment of anal fistulas with high-resolution subtraction MRfistulography: comparison with surgical findings. Magn Reson Imaging 19(1):91-98

366. Schäfer AO, Baumann T et al (2006) MRI for the detection of anorectal fistulas. Rofo 178(11):1095-1104

367. Schmiegel W, Pox C et al (2008) "S3-Leitlinie "Kolorektales Karzinom, ,..Z Gastroenterol 46:1-73

368. Scholefield JH, Berry DP et al (1997) Magnetic resonance imaging in the management of fistula in ano. Int J Colorectal Dis 12(5):276-279

369. Schouten WR, Zimmerman DD et al (1999) Transanal advancement flap repair of transsphincteric fistulas. Dis Colon Rectum 42(11):1419-1422 (discussion 1422-3)

370. Schulze B, Ho YH (2015) Management of complex anorectal fistulas with seton drainage plus partial fistulotomy and subsequent ligation of intersphincteric fistula tract (LIFT). Tech Coloproctol 19(2):89-95

371. Schurr MO, Prosst RL (2016) Comment on: Easy clip to treat anal fistula tracts: a word of caution by $\mathrm{M}$. Gautier et al. Int J Colorectal Dis 31(3):707-708

372. Schwandner 0 (2011) Obesity is a negative predictor of success after surgery for complex anal fistula. BMC Gastroenterol 11:61

373. Schwandner O (2011) „Perianale Fisteln." BDC online.

374. Schwandner O (2013) Video-assisted anal fistula treatment (VAAFT) combined with advancement flap repair in Crohn's disease. Tech Coloproctol 17(2):221-225

375. Schwandner O, Stadler F et al (2008) Initial experience on efficacy in closure of cryptoglandular and Crohn's transsphincteric fistulas by the use of the anal fistula plug. Int J Colorectal Dis 23(3):319-324

376. Schwandner T, Roblick MH et al (2009) Surgical treatment of complex anal fistulas with the anal 
fistula plug: a prospective, multicenter study. Dis Colon Rectum 52(9):1578-1583

377. Schwartz DA, Wiersema MJ et al (2001) A comparison of endoscopicultrasound, magnetic resonance imaging, and exam under anesthesia for evaluation of Crohn's perianal fistulas. Gastroenterology 121(5):1064-1072

378. Scott F, Seghal A et al (2014) Quality of patient information on the internet for the treatment of anal fistula and anal fissure. Tech Coloprocto 18:1181-1183

379. Seneviratne SA, Samarasekera DN et al (2009) Quality of life following surgery for recurrent fistula-in-ano. Tech Coloproctol 13(3):215-217

380. Sentovich SM (2001) Fibrin glue for all anal fistulas. J Gastrointest Surg 5(2):158-161

381. Sentovich SM (2003) Fibrin glue for anal fistulas: long-term results. Dis Colon Rectum 46(4):498-502

382. Seow-Choen F, Hay AJ et al (1992) Bacteriology of anal fistulae. Br JSurg 79(1):27-28

383. Seow-Choen F, Ho JM (1994) Histoanatomy of anal glands. Dis Colon Rectum 37(12):1215-1218

384. Seow-Choen F, Nicholls RJ (1992) Anal fistula. Br J Surg 79(3):197-205

385. Serour F, Gorenstein A (2006) Characteristics of perianal abscess and fistula-in-ano in healthy children. World J Surg 30(3):467-472

386. Shanwani A, Nor AM et al (2010) Ligation of the intersphincteric fistula tract (LIFT): a sphinctersaving technique for fistula-in-ano. Dis Colon Rectum 53(1):39-42

387. Sheikh P, Baakza A (2014) Management of Fistulain-Ano-The Current Evidence. Indian J Surg 76(6):482-486

388. Shemesh El, Kodner IJ et al (1988) Endorectal sliding flap repair of complicated anterior anoperineal fistulas. Dis Colon Rectum 31(1):22-24

389. Shouler PJ, Grimley RP et al (1986) Fistula-in-ano is usually simple to manage surgically. Int J Colorect Dis 1:113-115

390. Shukla HS (1991) Multicentric randomized controlled clinical trial of Kshaarasootra (Ayurvedic medicated thread) in the management of fistulain-ano. Indian Council of Medical Research... Indian JMed Res 94:177-185

391. Siddiqui MR, Ashrafian $\mathrm{H}$ et al (2012) A diagnostic accuracy meta-analysis of endoanal ultrasound and MRI for perianal fistula assessment. Dis Colon Rectum 55(5):576-585

392. Sierra EM, Villanueva Saenz E et al (2006) Mucinous adenocarcinoma associated with fistula in ano: report of a case. Tech Coloproctol 10(1):51-53

393. Sileri P, Cadeddu F et al (2011) Surgery for fistulain-ano in a specialist colorectal unit: a critical appraisal. BMC Gastroenterol 11:120

394. Sileri P, Franceschilli L et al (2011) Ligation of the intersphincteric fistula tract (LIFT) to treat anal fistula: early results from a prospective observational study. Tech Coloproctol 15(4):413-416

395. Sileri P, Franceschilli L et al (2011) Porcine dermal collagen matrix injection may enhance flap repair surgery for complex anal fistula. Int J Colorectal Dis 26(3):345-349

396. Sileri P, Giarratano G et al (2014) Ligation of the intersphincteric fistula tract (LIFT): a minimally invasive procedure for complex anal fistula: twoyear results of a prospective multicentric study. Surg Innov 21(5):476-480

397. Singer M, Cintron J et al (2005) Treatment of fistulas-in-ano with fibrin sealant in combination with intra-adhesive antibiotics and/or surgical closure of the internal fistula opening. Dis Colon Rectum 48(4):799-808
398. Sirany AM, Nygaard RM et al (2015) The ligation of the intersphincteric fistula tract procedure for ana fistula: a mixed bag of results. Dis Colon Rectum 58(6):604-612

399. Sirikurnpiboon S, Awapittaya B etal (2013) Ligation ofintersphinctericfistula tractand itsmodification: results from treatment of complex fistula. World J Gastrointest Surg 5(4):123-128

400. Sofic A, Beslic S et al (2010) MRI in evaluation of perianal fistulae. Radiol Oncol 44(4):220-227

401. Soltani A, Kaiser AM (2010) Endorectal advancement flap for cryptoglandular or Crohn's fistula-inano. Dis Colon Rectum 53(4):486-495

402. Song KH (2012) New techniques for treating an anal fistula. JKorean Soc Coloproctol 28(1):7-12

403. Song WL, Wang ZJ et al (2008) An anorecta fistula treatment with acellular extracellular matrix: a new technique. World J Gastroenterol 14(30):4791-4794

404. Stamatiadis A, Konstantinou E et al (2002) Frequency of operative trauma to anal sphincters: evaluation with endoanal ultrasound. Gastroente rolNurs 25(2):55-59

405. Stamos MJ, Snyder M et al (2015) Prospective multicenter study of a synthetic bioabsorbable anal fistula plug to treat cryptoglandular transsphincteric anal fistulas. Dis Colon Rectum 58(3):344-351

406. Steele SR, Kumar R et al (2011) Practice parameters for the management of perianal abscess and fistula-in-ano. Dis Colon Rectum 54(12):1465-1474

407. Steinemann D, Dindo D et al (2010) Pilonidalsinus und Analfistel: Indikationen und Methoden der chirurgischen Therapien. Gastroenterologe 5:308-317

408. Steinemann D, Dindo D et al (2011) Pilonidalsinus und Analfistel. coloproctology 33:160-170

409. Stelzner F (1959) Die anorektalen Fisteln. Springer Berlin, Heidelberg

410. Stelzner F (1992) Anatomisch bedingte diagnos tische und operationstechnische Probleme und Komplikationen in der Chirurgie am Anorektum. ZentralblChir 117:111-114

411. Stelzner F, Dietl H et al (1956) Ergebnisse bei Radikaloperationen von 143 Analfisteln (Kritik der einzeitigen Sphinctertrennung bei einoder mehrzeitigen Fisteloperationen). Chirurg 27(4):158-162

412. Stremitzer S, Riss S et al (2012) Repeat endorecta advancement flap after flap breakdown and recurrence of fistula-in-ano - is it an option? Colorectal Dis 14:1389. doi:10.1111/j.1463-1318. 2012.02990.x

413. Subasinghe D, Samarasekera DN (2010) Comparison of preoperative endoanal ultrasonography with intraoperative findings for fistula in ano. World JSurg 34(5):1123-1127

414. Subhas G, Gupta A et al (2010) Non-cutting setons for progressive migration of complex fistula tracts: a new spin on an old technique. Int J Colorectal Dis 26(6):793-798

415. Subhas G, Singh Bhullar J et al (2012) Setons in the treatment of anal fistula: review of variations in materials and techniques. Dig Surg 29(4):292-300

416. Sungurtekin U, Sungurtekin $\mathrm{H}$ et al (2004) Anocutaneous $\mathrm{V}-\mathrm{Y}$ advancement flap for the treatment of complex perianal fistula. Dis Colon Rectum 47(12):2178-2183

417. Swinscoe MT, Ventakasubramaniam AK et al (2005) Fibrin glue for fistula-in-ano: the evidence reviewed. Tech Coloproctol 9(2):89-94

418. Sygut A, Mik M et al (2010) How the location of the internal opening of anal fistulas affect the treatment results of primary transsphincteric fistulas. Langenbecks Arch Surg 395(8):1055-1059

419. Sygut A, Zajdel R et al (2007) Late results of treatment of anal fistulas. Colorectal Dis 9(2):151-158

420. Tan KK, Alsuwaigh R et al (2012) To LIFT or to flap? Which surgery to perform following seton insertion for high anal fistula? Dis Colon Rectum 55(12):1273-1277

421. Tan KK, LeePJ(2014) Early experience of reinforcing the ligation of the intersphincteric fistula tract procedure with a bioprosthetic graft (BioLIFT) for anal fistula. ANZ J Surg 84(4):280-283

422. Tan KK, Tan IJ et al (2011) The anatomy of failures following the ligation of intersphincteric tract technique for anal fistula: a review of 93 patients over 4 years. Dis Colon Rectum 54(11):1368-1372

423. Tasci I (2003) „The fistulectome: a new device for treatment of complex anal fistulas by" Core-Out "fistulectomy...DisColon Rectum46(11):1566-1571

424. Theerapol A, So BY et al (2002) Routine use of setons for the treatment of anal fistulae. Singapore Med J 43(6):305-307

425. Thekkinkattil DK, Botterill I et al (2009) Efficacy of the anal fistula plug in complex anorectal fistulae. Colorectal Dis 11(6):584-587

426. Thomson JP, Parks AG (1979) Anal abscesses and fistulas. Br JHosp Med 21(4):413-4-420-2 passim

427. Thomson JPS, Ross AHM (1989) Can the external anal sphincter be preserved in the treatment of trans-sphincteric fistula-in-ano. Int J Colorect Dis 4:247-250

428. Thomson WH, Fowler AL (2004) Direct appositional (no flap) closure of deep anal fistula. Colorectal Dis 6(1):32-36

429. Tinay OE, El-Bakry AA (2003) Treatment of chronic fistula-in-ano using commercial fibrin glue. Saudi Med J24(10):1116-1117

430. Tobisch A, Stelzner Set al (2012) Total fistulectomy with simple closure of the internal opening in the management of complex cryptoglandular fistulas: long-term results and functional outcome. Dis Colon Rectum 55(7):750-755

431. Tokunaga $Y$, Sasaki $H$ et al (2013) Clinical role of a modified seton technique for the treatment of trans-sphincteric and supra-sphincteric anal fistulas. Surg Today 43(3):245-248

432. Tomiyoshi SD, Dos Santos CH (2014) Effectiveness of the ligation of intersphincteric fistula tract (LIFT) in the treatment of anal fistula: initial results. Arq Bras Cir Dig 27(2):101-103

433. Torkzad MR, Karlbom U (2010) MRI for assessment of anal fistula. Insights Imaging 1(2):62-71

434. Toyonaga T, Matsushima M et al (2006) Postoperative urinary retention after surgery for benign anorectal disease: potential risk factors and strategy for prevention. Int J Colorectal Dis 21(7):676-682

435. Toyonaga T, Matsushima $M$ et al (2007) Nonsphincter splitting fistulectomy vs conventional fistulotomy for high trans-sphincteric fistula-inano: a prospective functional and manometric study. Int J Colorectal Dis 22(9):1097-1102

436. Toyonaga T, Tanaka Y et al (2008) Comparison of accuracy of physical examination and endoanal ultrasonography for preoperative assessment in patients with acute and chronic anal fistula. Tech Coloproctol 12(3):217-223

437. Tozer P, Rayment $\mathrm{N}$ et al (2015) Whot role do bacteria play in persisting fistula formation in idiopathic and Crohn's anal fistula? Colorectal Dis 17:235-241

438. Tozer P, Sala Set al (2013) Fistulotomy in the tertiary setting can achieve high rates of fistula cure with 
an acceptable risk of deterioration in continence. J Gastrointest Surg 17(11):1960-1965

439. Tsunoda A, Sada H et al (2013) Anal function after ligation of the intersphincteric fistula tract. Dis Colon Rectum 56(7):898-902

440. Tyler KM, Aarons CB et al (2007) Successful sphincter-sparing surgery for all anal fistulas. Dis Colon Rectum 50(10):1535-1539

441. Uribe N, Balciscueta Z et al (2015) "Core out"

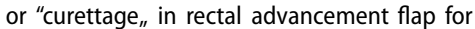
cryptoglandular anal fistula. Int J Colorectal Dis 30(5):613-619

442. Uribe N, Millan M et al (2007) Clinical and manometric results of endorectal advancement flaps for complex anal fistula. Int J Colorectal Dis 22(3):259-264

443. Ustynoski K, Rosen L et al (1990) Horseshoe abscess fistula. Seton treatment. Dis Colon Rectum 33(7):602-605

444. van der Hagen SJ, Baeten CG et al (2005) Staged mucosal advancement flap for the treatment of complex anal fistulas: pretreatment with noncutting Setons and in case of recurrent multiple abscesses a diverting stoma. Colorectal Dis 7(5):513-518

445. van der Hagen SJ, Baeten CG et al (2006) Longterm outcome following mucosal advancement flap for high perianal fistulas and fistulotomy for low perianal fistulas: recurrent perianal fistulas: failure of treatment or recurrent patient disease? Int JColorectal Dis 21(8):784-790

446. van der Hagen SJ, Baeten CG et al (2011) Autologous platelet-derived growth factors (platelet-rich plasma) as an adjunct to mucosal advancementflapin high cryptoglandularperianal fistulae: a pilot study. Colorectal Dis 13(2):215-218

447. van der Hagen SJ, Baeten CG et al (2011) Staged mucosal advancement flap versus staged fibrin sealant in the treatment of complex perianal fistulas. Gastroenterol Res Pract 2011:186350

448. van Koperen PJ, Bemelman WA et al (2008) The anal fistula plug versus the mucosal advancement flap for the treatment of anorectal fistula (PLUG trial). BMC Surg 8:11

449. van Koperen PJ, Bemelman WA et al (2011) The anal fistula plug treatment compared with the mucosal advancement flap for cryptoglandular high transsphincteric perianal fistula: a doubleblinded multicenter randomized trial. Dis Colon Rectum 54(4):387-393

450. van Koperen PJ, D’Hoore A et al (2008) Analer Fistelplug zum Verschluss komplizierter anorektaler Fisteln: eine prospektive Studie. coloproctology 30(3):159-164

451. van Koperen PJ, ten Kate FJ et al (2010) Histological identification of epithelium in perianal fistulae: a prospective study. Colorectal Dis 12(9):891-895

452. van Koperen PJ, Wind J et al (2008) Long-term functional outcome and risk factors for recurrence after surgical treatment for low and high perianal fistulas of cryptoglandular origin. Dis Colon Rectum 51(10):1475-1481

453. van Koperen PJ, Wind J et al (2008) Fibrin glue and transanal rectal advancement flap for high transshincteric perianal fistulas; is there any advantage? Int J Colorectal Dis 23:697-701

454. van Onkelen RS, Gosselink MP et al (2012) Is it possible to improve the outcome of transanal advancement flap repair for high transsphincteric fistulas by additional ligation of the intersphincteric fistula tract? Dis Colon Rectum 55(2):163-166

455. van Onkelen RS, Gosselink MP et al (2012) Ligation of the intersphincteric fistula tract in low transsphincteric fistula: a new technique to avoid fistulotomy. Colorectal Dis 14:587. doi:10.1111/ codi. 12030

456. van Onkelen RS, Gosselink MP et al (2013) Treatment of anal fistulas with high intersphincteric extension. Dis Colon Rectum 56(8):987-991

457. van Onkelen RS, Gosselink MP et al (2014) Predictors of outcome after transanal advancement flap repair for high transsphincteric fistulas. Dis Colon Rectum 57(8):1007-1011

458. van Tets WF, Kuijpers HC (1994) Continence disorders after anal fistulotomy. Dis Colon Rectum 37(12):1194-1197

459. van Tets WF, Kuijpers JH (1995) Seton treatment of perianal fistula with high anal or rectal opening. $\mathrm{Br}$ J Surg 82(7):895-897

460. van Tets WF, Kuijpers JH et al (1997) Influence of Parks' anal retractor on anal sphincter pressures. Dis Colon Rectum 40(9):1042-1045

461. Vasilevsky CA, Gordon PH (1984) The incidence of recurrent abscesses or fistula-in-ano following anorectal suppuration. Dis Colon Rectum 27(2):126-130

462. Vasilevsky CA, Gordon PH (1985) Results of treatment of fistula-in-ano. Dis Colon Rectum 28(4):225-231

463. Vatansev C, Alabaz $O$ et al (2007) A new seton type for the treatment of anal fistula. Dig Dis Sci 52(8):1920-1923

464. Venkatesh KS, Ramanujam P (1999) Fibrin glue application in the treatment of recurrent anorecta fistulas. Dios Colon Rectum 42:1136-1139

465. Vergara-Fernandez O, Espino-Urbina LA (2014) Ligation of intersphincteric fistula tract: What is the evidence in a review? World J Gastroenterol 19(40):6805-6813

466. Vial M, Pares D et al (2010) Faecal incontinence after seton treatment for anal fistulae with and without surgical division of internal anal sphincter: a systematic review. Colorectal Dis 12(3):172-178

467. Walega P, Romaniszyn Met al (2014) VAAFT: a new minimally invasive method in the diagnostics and treatment of anal fistulas - initial results. Pol Przegl Chir 86(1):7-10

468. WalfischS, Menachem Yetal (1997) Double setona new modified approach to high transsphincteric anal fistula. Dis Colon Rectum 40(6):731-732

469. Wallin UG, Mellgren AF et al (2012) Does ligation of the intersphincteric fistula tract raise the bar in fistula surgery? Dis Colon Rectum 55(11):1173-1178

470. Wang C, Lu JG et al (2012) Traditional Chinese surgical treatment for anal fistulae with secondary tracks and abscess. World J Gastroenterol 18(40):5702-5708

471. Wang D, Yang G et al (2014) Risk factors for anal fistula: a case-control study. Tech Coloproctol 18(7):635-639

472. Wang JY, Garcia-Aguilar J et al (2009) Treatment of transsphincteric anal fistulas: are fistula plugs an acceptable alternative? Dis Colon Rectum 52(4):692-697

473. WeberE, Buchmann P (1982) Eröffnung anorectaler Abszesse - mit oder ohne Fistelspaltung. Chirurg 53(4):270-272

474. Wedel T (2006) Der Beckenboden: Anatomische Grundlagen. Viszeralchirugie 41(3):153-162

475. Wedell J, Meier zu Eissen P et al (1987) Sliding flap advancement for the treatment of high level fistulae. Br J Surg 74(5):390-391

476. Weisman N, Abbas MA (2008) Prognostic value of endoanal ultrasound for fistula-in-ano: a retrospective analysis. Dis Colon Rectum 51(7):1089-1092
477. Weisman Rl, Orsay CP et al (1991) The role of fistulography in fistula-in-ano. Report of five cases. Dis Colon Rectum 34(2):181-184

478. West RL, Dwarkasing S et al (2004) Hydrogen peroxide-enhanced three-dimensional endoanal ultrasonography and endoanal magnetic resonance imaging in evaluating perianal fistulas: agreement and patient preference. Eur J Gastroenterol Hepatol 16(12):1319-1324

479. Westerterp M, Volkers NA et al (2003) Anal fistulotomy between Skylla and Charybdis. Colorectal Dis 5(6):549-551

480. Whiteford MH, Kilkenny J 3rd et al (2005) Practice parameters for the treatment of perianal abscess and fistula-in-ano (revised). Dis Colon Rectum 48(7):1337-1342

481. Wijekoon NS, Samarasekera DN (2010) The value of routine histopathological analysis in patients with fistula in-ano. Colorectal Dis 12:94-96

482. Wilhelm A (2011) A new technique for sphincterpreserving anal fistula repair using a novel radial emitting laser probe. Tech Coloproctol 15(4):445-449

483. Williams JG, Farrands PA et al (2007) The treatment of anal fistula: ACPGBI position statement. Colorectal Dis 9(Suppl4):18-50

484. Williams JG, MacLeod CA et al (1991) Seton treatment of high anal fistulae. $\mathrm{Br} J$ Surg 78(10):1159-1161

485. Witte ME, Klaase JM et al (2007) Fibrin glue treatment for simple and complex anal fistulas. Hepatogastroenterology 54(76):1071-1073

486. Yang BL, Shao WJ et al (2009) Perianal mucinous adenocarcinoma arising from chronic anorectal fistulae: a review from single institution. Int J Colorectal Dis 24(9):1001-1006

487. Yassin NA, Hammond TMetal (2013) Ligation of the intersphincteric fistula tract in the management of anal fistula. A systematic review. Colorectal Dis 15(5):527-535

488. Yeung JM, Simpson JA et al (2010) Fibrin glue for the treatment of fistulae in ano - a method worth sticking to? Colorectal Dis 12(4):363-366

489. Zaheer S, Reilly WT et al (1998) Urinary retention after operations for benign anorectal diseases. Dis Colon Rectum 41(6):696-704

490. Zanotti C, Martinez-Puente C et al (2007) An assessment of the incidence of fistula-in-ano in four countries of the European Union. Int J Colorectal Dis 22(12):1459-1462

491. Zbar AP (2009) David Henry Goodsall: reassessment of the rule. Tech Coloproctol 13(3):185-188

492. Zbar AP (2010) Experience with Staged Mucosal Advancement Anoplasty for High Transsphincteric Fistula-in-Ano. West Indian Med J 56(5):446-450

493. Zbar AP (2012) „Video-assisted anal fistula treatment (VAAFT): a novel sphincter-saving procedure to repair complex anal fistulas" by Piercarlo Meinero and Lorenzo Mori. Tech Coloproctol 15(4):423-424

494. Zbar AP, Armitage NC (2006) Complex perirectal sepsis: clinical classification and imaging. Tech Coloproctol 10(2):83-93

495. Zbar AP, Oyetunji RO et al (2006) Transperineal versus hydrogen peroxide-enhanced endoanal ultrasonography in never operated and recurrent cryptogenic fistula-in-ano: a pilot study. Tech Coloproctol 10(4):297-302

496. Zbar AP, Ramesh J et al (2003) Conventional cutting vs. internal anal sphincter-preserving seton for high trans-sphincteric fistula: a prospective randomized manometric and clinical trial. Tech Coloproctol 7(2):89-94 
497. Zhang JT, Zhou WL et al (2011) New type of seton with irrigating tube for the treatment of high complex anal fistula: a simple and effective instrument. J Int Med Res 39(6):2414-2420

498. Zhu R, Shen Let al (2014) A new minimally invasive treatment for anal fistula. Front Med 9(1):77-81

499. Zimmerman DD, Delemarre JB et al (2003) Smoking affects the outcome of transanal mucosal advancement flap repair of trans-sphincteric fistulas. Br JSurg 90(3):351-354

500. Zimmerman DD, Gosselink MP et al (2003) Impact of two different types of anal retractor on fecal continence after fistula repair: a prospective, randomized, clinical trial. Dis Colon Rectum 46(12):1674-1679

501. ZimmermanDD, GosselinkMPetal (2005) Smoking impairs rectal mucosal bloodflow - a pilot study: possible implications for transanal advancement flap repair. Dis Colon Rectum 48(6):1228-1232

502. Zimmerman DDE, Briel JW et al (2001) Anocutaneous advancement flap repair of transsphincteric fistulas. Dis Colon Rectum 44:1474-1480

503. Zmora O, Mizrahi N et al (2003) Fibrin glue sealing in the treatment of perineal fistulas. Dis Colon Rectum 46(5):584-589

504. Zubaidi A, Al-Obeed O (2009) Anal fistula plug in high fistula-in-ano: an early Saudi experience. Dis Colon Rectum 52(9):1584-1588

\section{J. Köbberling \\ Behandlungsfehler und Arzthaftung}

Praktische Hinweise für Ärzte und Patienten

Berlin: De Gruyter 2016, 128 S., 2 Abb., (ISBN: 978-3-11-047675-0), Hardcover 39,95 EUR

Das Buch Behandlungsfehler und Arzthaftung. Praktische Hinweise für Ärzte und Patienten von Johannes Köbberling (De Gruyter, 2016) gibt wichtige Praxisanleitungen zur Versachlichung des Arzt-Patienten-Verhältnisses bei vermuteten Behandlungsfehlern und ist daher ein nützlicher Leitfaden. Der Autor, Prof. Dr. Johannes Köbberling, war langjährig klinisch tätiger Internist, hat sich in vielfältigen Funktionen mit Fragen der Patientensicherheit befasst und ist $u$. a. Mitglied der Gutachterkommission für ärztliche Behandlungsfehler der Ärztekammer Nordrhein. Kritisch durchgesehen und um ein Vorwort ergänzt wurde das Buch von dem Medizinrechtler Prof. Peter Wolfgang Gaidzik. Er attestiert Köbberling, dass es inm gelungen ist, die komplexe Materie des Arzthaftungsrechts für den juristischen Laien verständlich werden zu lassen.

Wesentliches Anliegen des Buches ist das Eintreten für eine neue Kultur im Gesundheitswesen, in der Fehler nicht verleugnet oder gar vertuscht, sondern aktiv aufgegriffen werden, um daraus für die Zukunft zu lernen. Es ist sowohl als Hilfestellung für Ärzte gedacht, die sich mit Behandlungsfehlervorwürfen konfrontiert sehen, als auch als Informationsquelle und Handreichung für Patienten und Angehörige, wenn sie einen Behandlungsfehler vermuten. Dabei legt Köbberling Wert auf die Tatsache, dass Fehler zum Wesen des Menschen gehören und also auch im ärztlichen Handeln vorkommen entscheidend ist aber der Umgang damit. „Verantwortungsvolle Ärzte versuchen (...), aus ihren Fehlern zu lernen. Damit bleiben sie trotz begangenen Fehlers gute Ärzte." (S. 1). Nach einem Kapitel über Fehlerkultur und Fehlervermeidungsstrategien widmet sich Köbberling in neun gut gegliederten und informativen Kapiteln einzelnen Aspekten des Arzthaftungsrechts: Er gibt einen Einblick in die rechtlichen Grundlagen, definiert Behandlungsfehler und ihre Ursachen, erklärt den Begriff des Schadens und des Schadensausgleichs, erörtert das Thema Beweislast und Beweiserleichterungen, erläutert ausführlich die Besonderheiten von
Diagnosefehlern, widmet sich den Themen Aufklärung, Einwilligung und Dokumentation, um schließlich auf das Stichwort Sachverständigengutachten einzugehen. Alle Kapite werden durch zahlreiche authentische Praxisbeispiele, vornehmlich aus der eigenen Gutachtertätigkeit, ergänzt und erhalten dadurch Lebendigkeit und Anschaulichkeit. Der praktische Wert des Buches wird dadurch erhöht, dass die wichtigsten Kernsätze in farbig markierten Blöcken optisch hervorgehoben werden. Zudem beinhaltet auch das Kapitel über die Gutachterkommission unter Angabe von Adressen und Verfahrensabläufen einen konkreten Nutzwert. Was das Buch aber besonders auszeichnet, sind die beiden Schlusskapitel „Wie verhalten Sie sich als Ärztin oder Arzt bei Behandlungsfehlervorwürfen?" und "Wie gehen Sie als Patient bei möglichen Behandlungsfehlern vor?", in denen Köbberling empathisch die Perspektive des jeweiligen Betroffenen einnimmt und konkrete und konstruktive Lösungen aufzeigt.

Das Buch ermöglicht den medizinrechtlich abgesicherten Blick über den Tellerrand des klinischen Alltags, bietet konkrete Handlungsempfehlungen und ebnet den Weg in eine adäquate Fehlerkultur.

C. Güsgen (Koblenz) 